Efficacité de l'Approche de l'Enseignement Différencié pour Remédier aux Difficultés Linguistiques en FLÉ chez les Élèves du Cycle Préparatoire.

Étude préparée par

Dr. Abdel Nasser Chérif Mohammed

Prof-adjoint de la Didactique de FLÉ

Université du Fayoum.

Le Caire 2017 


\section{Efficacité de l'Approche de l'Enseignement Différencié pour Remédier aux Difficultés Linguistiques en FLÉ chez les Élèves du Cycle Préparatoire.}

\section{Dr. Abdel Nasser Chérif Mohammed.}

مستخلص باللغة العربية.

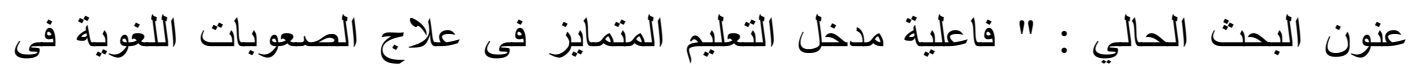

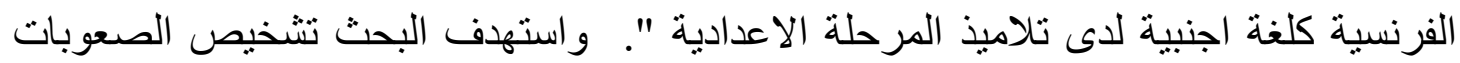

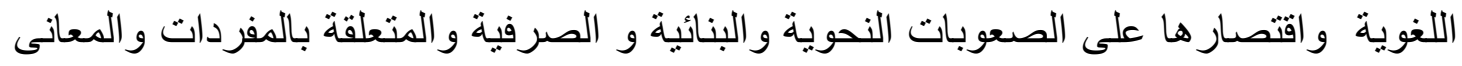

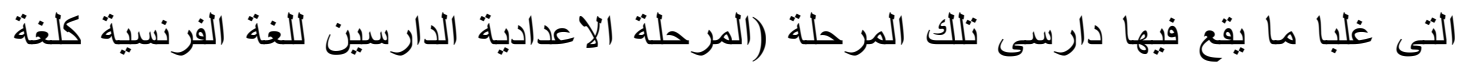

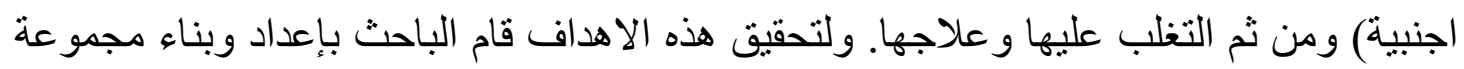

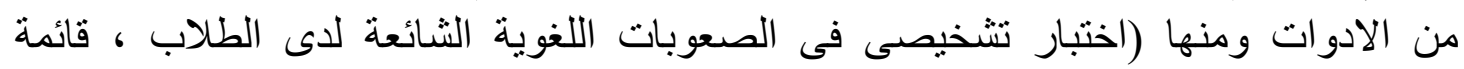

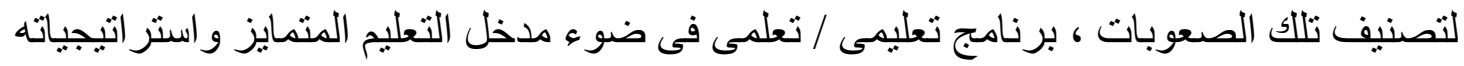

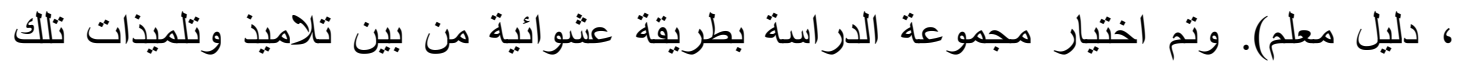

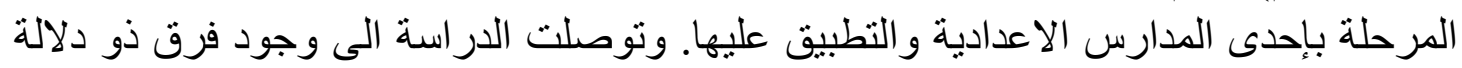

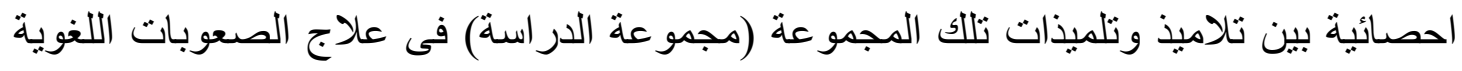

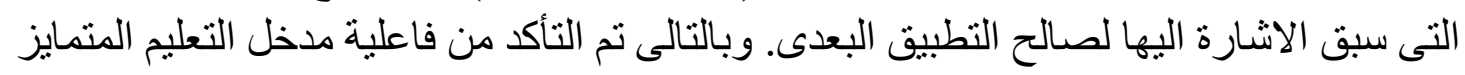

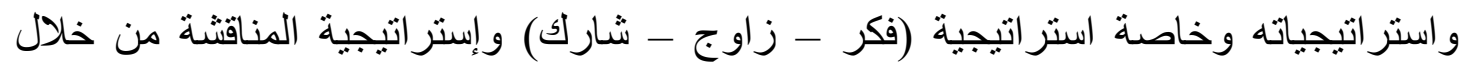

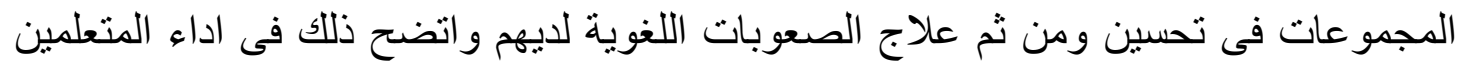

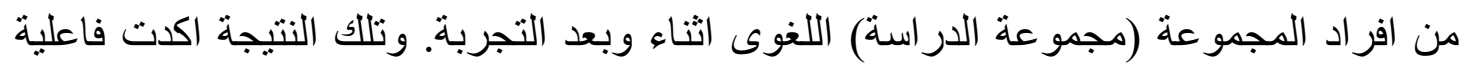

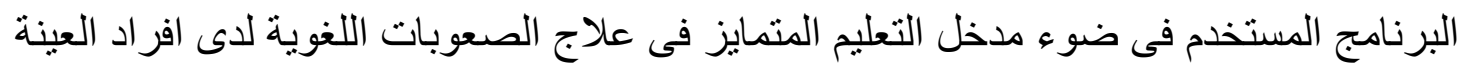

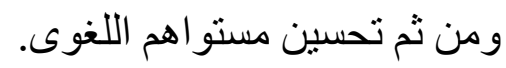

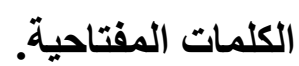

مدخل التعليم المتمايز ـ المهارات اللغوية ـ الصعوبات اللغوية. 


\section{Résumé en langue française.}

L'étude actuelle est intitulée : «Efficacité de l'approche de l'enseignement différencié pour remédier aux difficultés linguistiques du cycle préparatoire». Elle a pour objectif de diagnostiquer les difficultés linguistiques. Celles-ci se sont limitées aux difficultés grammaticales, structurales, morphologiques, lexicales et sémantiques propres à ce niveau d'apprentissage afin de les surmonter et d' y remédier. Pour bien atteindre ces objectifs, on a élaboré quelques outils tels qu'un test de difficultés linguistiques (employé comme un test de diagnostic et test de progrès), une typologie de difficultés, un programme élaboré d'après les apports de l'enseignement différencié et ses stratégies notamment la stratégie de (Penser-Comparer-Partager) et celle de la discussion en groupes coopératifs, et un guide pédagogique. On a choisi un groupe d'étude parmi les apprenants(es) de FLÉ au cycle préparatoire d'une école dans le gouvernorat du Caire pour l'aspect de l'intervention. L'étude a confirmé qu'il existe une différence significative entre les moyennes des notes des apprenants(es) de FLÉ du pré - post test de difficultés linguistiques en faveur du post test. Ce résultat a confirmé l'efficacité du programme élaboré dans le domaine de traiter les difficultés linguistiques chez les sujets du groupe de l'étude.

\section{Mots clés.}

L'approche de l'enseignement différencié - les compétences linguistiques - les difficultés linguistiques. 


\section{Introduction.}

L'enseignement / apprentissage des différentes compétences des langues étrangères vivantes en général et de la langue française en particulier cherche à développer au fur et à mesure chez les apprenants(es) des structures langagières et des compétences linguistiques (compétences syntaxiques, grammaticales, structurales, morphologiques, lexicales et sémantiques) indispensables à être employées pour comprendre, se faire comprendre et pour s'exprimer correctement dans une société quelconque. Autrement dit, les compétences linguistiques se pratiquent à travers le fonctionnement correct d'un nombre de règles variées (règles grammaticales, structurales, syntaxiques, morphologiques, lexicales et sémantiques) et ces règles linguistiques diversifiées se varient selon les visions différentes des théories linguistiques successives. Également les structures linguistiques sont employées à travers les différentes théories et approches psychopédagogiques et visent à atteindre des objectifs socio-pédagogiques.

On doit noter qu'un temps considérable de telle ou telle séance est consacré au processus de l'enseignement / apprentissage de n'importe quelle langue enseignée ou bien langue étrangère (LÉ) pour chercher la normativité linguistique des phrases composées par les apprenants(es) de cette langue; les enseignants(es) déploient un effort énorme pour rendre ces phrases linguistiquement admissibles. Ils corrigent ces phrases du point de vue linguistique; du point de vue grammatical, structural, morphologique, phonologique, orthographique, lexical et sémantique. (Vicente J., 2016). En d'autres termes, on consacre plusieurs heures du 
travail en classe de langue pour corriger les erreurs et les fautes commises par les enseignés(es).

À cet effet, Oscar Valenzuela (Valenzuela O., 2010) a certifié que l'enseignement / apprentissage de n'importe quelle langue étrangère loin de son environnement naturel suscite plusieurs difficultés soit au niveau de la motivation des apprenants(es) de cette langue comme langue étrangère, soit au niveau de sa maîtrise. Les apprenants(es), d'après lui, abandonnent au fur et à mesure la langue lors de quitter la salle de classe et ne la rappellent qu'au moment où ils y retournent de nouveau. Ils / elles la pratiquent donc dans un temps limité de façon quotidienne et d'ici on touche un type d'énormes difficultés en face de tels(telles) apprenants(es).

Également les enseignants(es) des langues étrangères doivent prendre en considération de développer leurs propres connaissances linguistiques au même titre que leurs savoirs épistémologiques en linguistique et en psychopédagogie pour développer leur travail dans les salles de classe pour l'objectif d'avoir la capacité de développer les différentes compétences linguistiques et pédagogiques chez leurs apprenants(es) comme les médecins qui doivent prendre en considération le développement de leurs savoirs en biologie et en médecine pour développer leur travail afin de bien soigner leurs malades. À ce propos Luce Brossard (Brossard L., 1999) a mentionné qu'il est illogique que les enseignants(es) ne développent pas leurs connaissances par tout ce qui est d'avant-garde dans le domaine de la psychologie cognitive lors du travail dans les classes de langue. Il est donc nécessaire de prendre en compte les données nouvelles venues de la psychologie cognitive. 
Plusieurs psychopédagogues comme Claude Paquette (Paquette C., 1999) et Michelyne Lortie-Paquette (Lortie-Paquette M., 1999) ont été convaincu que si les enseignants(es) improvisent dans leurs salles de classe lors du travail pour développer telle ou telle compétence langagière, ils n'arrivent sans doute à rien au sein de leur travail pour développer ces compétences langagières chez leurs apprenants(es) en général et pour développer les compétences linguistiques en particulier. Il est donc obligatoire de traiter les compétences linguistiques de façon correcte selon les nouvelles tendances psychologiques.

Plusieurs linguistes comme Moshé Starets (Starets M., 2008 : P. 210) ont assuré que la langue en général soit maternelle soit étrangère ne s'enseigne de façon isolée mais qu'à travers des structures linguistiques. Cette confirmation de Moshé Starets assure l'importance des formes linguistiques (des règlements grammaticaux, structuraux, syntaxiques, morphologiques, lexiques et sémantiques) dans le domaine de la didactique de FLÉ. À cette perspective Moshé Starets a cité que «Les prépositions dénuées de sens dans certains contextes, les verbes colloqués, les idiotismes, entre autres, s'enseignent par l'habituation. Des mots, de faits de grammaire s'enseignent par l'analyse. L'enseignement d'une langue doit, par conséquent, conjuguer l'analyse des faits de langue et l'habituation à les employer dans des situations aussi authentiques que possible». Cela veut dire que lorsqu'on cherche à développer les compétences linguistiques, on doit suivre les mécanismes assidus des théories psychologiques pour les pratiquer correctement. 
Il est naturel que la linguistique générale englobant les règlements grammaticaux, syntaxiques, morphologiques, lexicaux et sémantiques dans des structures linguistiques correctes tient une place importante dans le processus de la didactique de FLÉ soit au niveau de la compréhension orale et écrite, soit au niveau de la production orale et écrite; il est bien connu pour tout le monde que lorsque l'on voudrais comprendre les messages oralement destinés ou bien par écrit, on doit maîtriser la nature de structurer les phrases françaises suivant les règlements grammaticaux, syntaxiques, morphologiques, lexicaux et sémantiques corrects et lorsque l'on voudrais s'exprimer correctement, on doit maîtriser également la nature de structurer les phrases françaises suivant les mêmes règlements. Les compétences linguistiques et leurs difficultés relatives tiennent donc leur place essentielle et centrale dans l'enseignement /apprentissage de FLÉ. Selon plusieurs linguistes français tels que (Richterich R. \& ., Suter B., 1981), (Chiss J.-L. et Meleuc S. 2001) et (Chiss J.-L., 2002), la grammaire comme un aspect linguistique garde une place inévitable soit dans les manuels de la didactique de français langue maternelle (FLM) soit dans les programmes de la didactique de français langue étrangère (FLÉ), également dans leurs travaux propres au même titre dans les programmes des institutions où ils travaillent. Ils ont confirmé de façon directe ou indirecte qu'on ne peut pas faire comprendre ou faire produire les apprenants(es) de presque tous les niveaux d'apprentissage en neutralisant les règlements linguistiques (grammaticaux, structuraux, morpho syntaxiques, morphologiques, lexicaux et sémantiques). Autrement dit, et suivant cette vision des linguistes déjà mentionnés, lorsque les apprenants(es) se mettent à comprendre un texte donné ou 
bien à produire une tâche linguistique, ils(elles) doivent perfectionner les règles linguistiques pour éviter de tomber en fautes, lapsus ou erreurs.

Du point de vue linguistique, les chercheurs(euses) en didactique des langues travaillent au et à mesure pour aider les apprenants(es) de LVÉ voire de FLÉ de tous les niveaux d'apprentissage même les apprenants(es) ayant un niveau avancé à mieux perfectionner les compétences linguistiques mais il est observable que plusieurs difficultés persistent entraver dans les performances écrites ou orales de ces apprenants(es) à cause de l'interférence des compétences de la langue maternelle avec celles de la langue cible. D'autres difficultés sont revenues aux particularités de la langue cible. Cette langue cible est, dans la plupart du temps et d'après un nombre énorme de linguistes tels que Maïa Grégoire et Alina Kostucki (Grégoire M., et Kostucki A., 2012), mal perçue par les apprenants(es) malgré les travaux intensifs déployés par les enseignants(es) dans les salles de classe.

Ferdinand de Saussure (De Saussure F., 1972) a établi une certitude linguistique essentielle et en même temps élémentaire; ce principe saussurien était que la langue constitue une structure sur laquelle on peut fonder les autres éléments linguistiques. Cela veut dire que s'on voudrais enseigner /apprendre une langue de façon purement correcte, saine et sans difficultés, on doit développer ses compétences linguistiques chez nos apprenants /apprenantes. On enseigne cette langue comme des structures correctes du point de vue grammatical, structural, morphologique, lexical et sémantique. 
À ce propos Moshé Starets (Starets M., 2008) a confirmé que l'apprenant(e) a formé ses phrases de façon correcte en respectant les règles syntaxiques, morphologiques et grammaticales et a attribué le sens de ses phrases en respectant les règlements de la sémantique. Cela veut dire que les compétences linguistiques en gros constituent des bases solides pour bien étudier et bien maîtriser la langue soit au niveau du code écrit, soit au niveau du code oral; la langue ne constitue que des structures linguistiques employées pour distribuer des messages faciles à comprendre oralement ou par écrit. Alors si l'on respecte les règlements linguistiques ou bien si l'on perfectionnent ces règles, on peut, d'une façon ou d'une autre éviter la majorité des difficultés et des obstacles dits linguistiques qui sont probablement en face.

À la même perspective plusieurs colloques, tels que celui qui est tenu à l'école de français moderne de l'université de Lausanne (ÉFM) en Avril 2002, ont essayé de trouver réponses à des questions telles que Quelle est la place de la grammaire dans le processus de l'enseignement /apprentissage du français ?, Quel genre de grammaire faut-il enseigner?, Quel est son rôle au sein des échanges communicatifs dans et hors de la classe de langue? et Quel est son rôle dans les autres compétences soit de compréhension orale et écrite, soit de production orale et écrite?. Les chercheurs ont dirigé des sessions de longs et profonds débats pour non pas uniquement répondre à de telles questions posées mais également à tracer des pistes claires pour les futurs enseignants(es) et pour les didacticiens dans le domaine de la didactique de FLÉ. Ce débat a vraiment cassé les confiances solides de tels enseignants(es) au même titre des didacticiens (didacticiennes), et des chercheurs (chercheuses) 
auprès de la didactique de la grammaire et de la structure langagières de FLÉ. On a ajouté aussi une autre dimension à travers l'étude en cours, c'est de quelle manière la grammaire avec les autres aspects de compétences linguistiques sont-ils enseignés? et sur les apports sousjacents de quelle approche sont-ils enseignés de façon parfaite pour éviter leurs difficultés relatives?

En résumé, on a une réalité solide; la culture linguistique en général et en particulier la culture grammaticale, structurale, morphologique, lexicale et sémantique constituent une culture préalable dans les classes de la langue française comme langue étrangère et plusieurs linguistes tels que (Chiss J.-L., 2002), (Leeman D., 2002) restent les partisans de ce point de vue; on n'imagine pas une classe de langue sans enseigner les règles linguistiques de telle ou telle langue cible même s'on emploie des approches didactiques d'avant-garde. À cette perspective, quelques études antérieures telles que l'étude de Nicolas Dalmasso (Dalmasso N., 2009) où le chercheur a confirmé que les résultats obtenus montrent une corrélation positive entre la compétence grammaticale et la compétence pragmatique (la compétence d'usage) et l'étude de Danielle Leeman (Leeman D., 2002) où le chercheur a expliqué comment la culture grammaticale de l'enseignant(e) l'amène à développer les autres compétences de l'apprenant(e) et ainsi à développer chez lui / elle la curiosité, l'esprit de découverte, l'esprit créatif et critique à travers les exercices variés présentés au sein de la classe de langue. Ces études ont montré qu'il existe une corrélation étroite entre le développement des compétences linguistiques notamment les compétences grammaticales et structurales et les autres compétences de FLÉ. 
Des approches didactiques ont déjà traité le thème des difficultés linguistiques comme l'approche communicative, actionnelle, interactionniste ...etc. On a une conviction solide que s'on emploie d'autres approches et d'autres stratégies dites nouvelles telles que l'approche de l'enseignement différencié et ses mécanismes du travail peuvent améliorer la situation de la didactique des compétences linguistiques et leurs difficultés relatives sur le plan de l'enseignement / apprentissage de FLÉ.

Les apprenants(es) de telle ou telle matière scientifique sont bien entendu différents(es) voire hétérogènes par leurs niveaux académiques, leurs motivations, leurs acquis, leurs savoirs, leurs savoir-faire, leurs rythmes d'apprentissage, leurs intérêts, leurs profils pédagogiques. Or, on peut envisager des situations d'enseignement /apprentissage hétérogènes. Ces différences individuelles sont les causes essentielles derrières l'existence des difficultés linguistiques de quelques apprenants(es). Face à ces situations hétérogènes, Philippe Meirieu (Meirieu P., 1990) a mis l'accent sur les rôles de l'enseignant(e). Ces rôles d'après Meirieu et d'autres chercheurs(euses) en pédagogie différenciée, se résument de proposer des activités, des situations d'apprentissage, de guider les apprenants(es) vers la solution de leurs situations problématiques, d'observer, de présenter les supports en temps de difficultés et d'aider les apprenants(es) à surmonter leurs difficultés.

La majorité des pédagogues et des méthodologues sont presque d'accord sur le concept de l'approche de l'enseignement différencié. Celleci consiste à mettre à la disposition de chaque apprenant(e) des dispositifs convenables au niveau intellectuel et cognitif de chacun(e). Cette 
approche donne donc l'occasion à chaque apprenant(e) de se progresser individuellement; l'approche donne l'enseignant(e) l'occasion de prendre en compte les différences individuelles de leurs apprenants(es) en classe de langue et cela nécessite que les enseignants(es) varient leurs stratégies d'enseignement/ apprentissage et leurs activités présentées à leurs apprenants(es). Cela signifie que chaque établissement scolaire doit avoir des dispositifs et des programmes variés sinon il doit pousser les enseignants(es) de FLÉ à varier leurs activités et leurs stratégies du travail lors d'enseigner leurs programmes et leurs dispositifs. Philippe Perrenoud (Perrenoud Ph., 1993) a montré à ce propos que le processus de différenciation a pour objet de lutter contre l'échec scolaire.

Également, plusieurs spécialistes en pédagogie tels que Andrea Prupas et Silvia Patella (Prupas A. et Patella S., 2007) ont déterminé ce qu'on désigne par l'approche de l'enseignement différencié. Ils voient qu'elle prend en considération les intérêts, la préparation et le style de chaque apprenant(e). ces chercheurs ont ajouté qu'on doit tenir compte des caractéristiques et des besoins uniques et propres de chacun(e) de ces apprenants(es). D'après eux, c'est obligatoire à un établissement scolaire de diversifier ses dispositifs suivant les intérêts, les besoins uniques et caractéristiques de chacun(e) des apprenants(es).

Stéphanie Descampe et autres ( Descampe S., et al., 2014) ont adopté la définition du concept du décret de la communauté française de Belgique qui dit : l'approche de la pédagogie différenciée est comme «une démarche d'enseignement qui consiste à varier les méthodes pour tenir compte de l'hétérogénéité des classes ainsi que de la diversité des modes et des besoins d'apprentissage des élèves». Ici, les chercheurs à travers 
cette définition ont insisté sur la variété des méthodes d'enseignement adoptées pour faire face à des classes hétérogènes avec des besoins et des styles diversifiés des apprenants(es).

À la lueur de son mémoire intitulé «Comment mettre en place une pédagogie différenciée au sein d'une classe afin d'assurer les apprentissages auprès de chaque enfant ?», Geneviève Chanay (Chanay G., S. D.) a présenté une définition opérationnelle du terme en focalisant sur des démarches menées par les enseignants(es) afin d'atteindre un objectif donné avec les apprenants(es) d'une même classe quel que soit leur diversité. Il a expliqué que ces enseignants(es) emploient des voies différentes et adaptées aux particularités de chacun€ de ces apprenants(es) pour arriver aux mêmes fins à travers un panel d'outils. Cette définition de Geneviève est plus profonde dans la mesure de pousser ou bien d'entraîner les maîtres-enseignants et enseignantes à employer des stratégies et des outils variés lors de l'enseignement / apprentissage d'un même programme. La question selon la chercheuse n'est pas à élaborer des programmes ou bien des dispositifs diversifiés mais d'employer des activités, des tâches, des outils diversifiés pour enseigner le même programme et cela ce qui est recherché à travers l'étude en cours. On cherche donc à employer des stratégies et des activités diversifiées basées sur les apports sous-jacent de l'approche de l'enseignement différencié et ses mécanismes du travail pour remédier aux difficultés linguistiques communes chez les écoliers/ écolières de FLÉ au cycle préparatoire. 


\section{Position du problème.}

On se sent le problème de l'étude actuelle à travers les points cidessous :-

- Plusieurs études antérieures dans le domaine de l'emploi de l'approche de l'enseignement différencié telles que l'étude de Geneviève Chanay (Chanay G., S. D.), celle de Simpkins et autres (Simpkins et al., 2009) et celle de Stéphanie Descampe et autres (Descampe S., et al., 2014) ont recommandé qu'on doit l'employer pour développer les différentes compétences de l'enseignement / apprentissage en général et de FLÉ en particulier pour surmonter les difficultés linguistiques appropriées à chaque apprenant/ apprenante. On doit montrer que parmi les difficultés linguistiques communes de FLÉ, on trouve des difficultés structurales, grammaticales, syntaxiques, morphologiques, lexicales et sémantiques. S'on néglige des telles difficultés, on ne peut ni lire ni écrire la langue. Autrement dit, s'on ne se conscientise pas de règlements structuraux, grammaticaux, morphologiques, lexicaux et sémantiques, on ne peut pas ni comprendre oralement et par écrit les messages destinés en français, ni produire oralement et par écrit des phrases ou bien des paragraphes voire des textes en français.

- D'autres études antérieures dans le domaine de la linguistique en général et notamment de la grammaire, de la structure, de la morphologie, du lexique, de la sémantique telles que l'étude de (Richterich R. \& ., Suter B., 1981), celle de (Chiss J.-L. et Meleuc S. 2001) et celle de ( Chiss J.-L., 2002) ont présenté des visions analytiques et théoriques à ce propos et ont assuré que la culture 
linguistique en général et la culture grammaticale persiste quel que soit la méthodologie suivie pour bien rédiger au niveau de l'écrit et bien prononcer au niveau de l'oral. Ces études ont présenté des analyses détaillées pour confirmer l'importance de la linguistique.

- Un nombre énorme d'études antérieures telles que l'étude de Rémy Porquier (Porquier R., 1977), celle d'Anna Doquin (Doquin A., 2008), celle d'Ana Valverde Mateos (Valverde-Mateos A., 2012), celle de Nahla Chellouai (Nahla Chellouai, 2013), celle de Judith Vicente (Vicente J., 2016) focalisent sur les difficultés et les erreurs dans le domaine de l'enseignement / apprentissage de FLÉ. Ces études ont mentionné qu'on doit prendre en compte la normativité linguistique de la langue étrangère en général et plus particulièrement la langue française comme langue étrangère.

- Un nombre énorme de linguistes français tels que Maïa Grégoire et Alina Kostucki (Grégoire M., et Kostucki A., 2012) ont vu que quelques unes des difficultés linguistiques en français sont dues dans la plupart du temps aux interférences avec la langue d'origine (la langue maternelle) et aux particularités mal perçues par les apprenants(es) de la langue française; celles-ci quittent une influence considérable sur la communication parmi les sujets débutants. Si l'on n'est pas conscient de telles particularités, les messages destinés oralement ou par écrit ne sont pas arrivés de façon correcte.

- Des colloques nombreux autour du monde francophone tels que celui de l'université de Lausanne (ÉFM) tenu en Avril 2002 ont confirmé que les compétences linguistiques sont essentielles et on doit les traiter par des stratégies et des approches nouvelles. Et 
d'autre part des conférences telles que la conférence du conseil national d'évaluation du système scolaire tenue en 2017 (Forget A., 2017) ont montré qu'on doit adopter largement, lors de l'enseignement /apprentissage des compétences langagières l'approche de l'enseignement différencié et ses mécanismes du travail.

- Le chercheur a observé, comme expérience personnelle, que les apprenants(es) de FLÉ de la $2^{\text {ème }}$ année du cycle préparatoire commettent de façon répétée des erreurs grammaticales, structurales, morphologiques, lexiques et sémantiques lors de leur performance écrite au même titre que celle de l'oral. Cela s'est répété plusieurs reprises dans les tests de performance linguistique hebdomadaire et mensuel et les lacunes communes se sont résumées dans :-

- L'emploi des connecteurs au sein des phrases simples comme (et ou - mais ...etc.);

- L'emploi des connecteurs au sein des phrases composées comme (tandis que - alors que - bien que - pour que - afin que...etc.);

- L'art de bien conjuguer les verbes couramment employés, réguliers ou irréguliers aux modes et aux temps convenables pour communiquer dans les situations de la vie courante;

- L'emploi correct des adjectifs, des adverbes, des adverbes composés, des pronoms personnels et des prépositions de base au sein des phrases françaises;

- L'emploi des morphèmes grammaticaux et lexicaux de base; 
- Le choix du vocabulaire adéquat à des situations et à des thématiques de la vie courante pour s'exprimer correctement dans ces types de situations;

- L'emploi des synonymes et des antonymes au sein des phrases composées pour accéder simplement au sens.

- Pour s'assurer de telles difficultés, on a mené une étude de découverte où on a appliqué un test de difficultés linguistiques communes pour diagnostiquer scientifiquement ces types difficultés et cette étude a mis l'accent sur les difficultés communes dans le tableau suivant:-

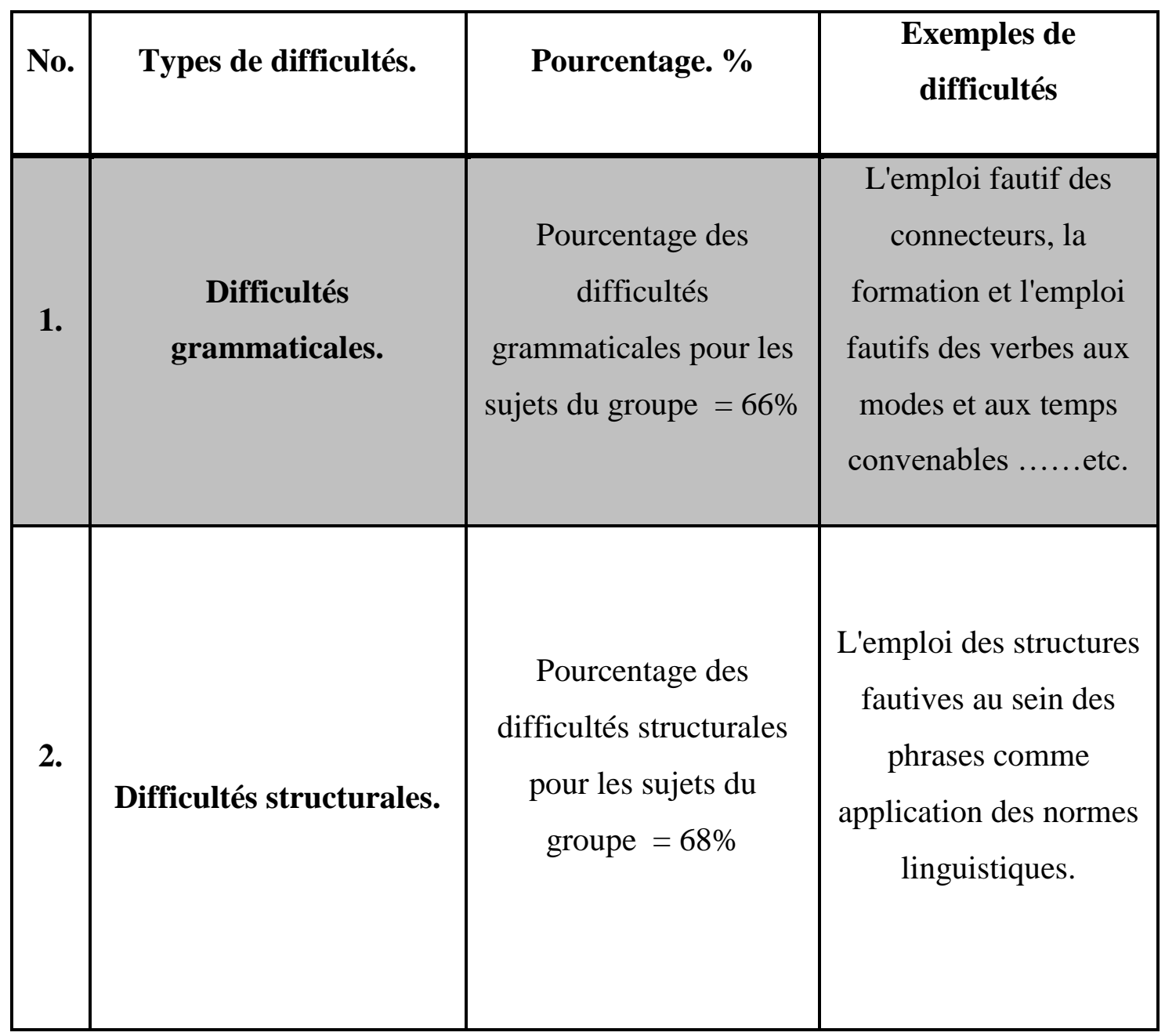




\begin{tabular}{|c|c|c|c|}
\hline 3. & $\begin{array}{c}\text { Difficultés } \\
\text { morphologiques. }\end{array}$ & $\begin{array}{c}\text { Pourcentage des } \\
\text { difficultés } \\
\text { morphologiques pour } \\
\text { les sujets du groupe = } \\
73 \%\end{array}$ & $\begin{array}{l}\text { L'emploi fautif des } \\
\text { morphèmes } \\
\text { grammaticaux comme } \\
\text { les articles définis et } \\
\text { indéfinis, les articles } \\
\text { partitifs....etc., et des } \\
\text { morphèmes lexicaux. }\end{array}$ \\
\hline 4. & Difficultés lexicales. & $\begin{array}{c}\text { Pourcentage des } \\
\text { difficultés lexicales pour } \\
\text { les sujets du groupe }= \\
76 \%\end{array}$ & $\begin{array}{c}\text { L'emploi fautif du } \\
\text { vocabulaire dans les } \\
\text { différentes situations } \\
\text { de la vie. }\end{array}$ \\
\hline 5. & Difficultés sémantiques. & $\begin{array}{c}\text { Pourcentage des } \\
\text { difficultés sémantiques } \\
\text { pour les sujets du } \\
\text { groupe }=79 \%\end{array}$ & $\begin{array}{l}\text { L'emploi fautif des } \\
\text { synonymes et des } \\
\text { antonymes dans les } \\
\text { phrases en français. }\end{array}$ \\
\hline
\end{tabular}

\section{La Problématique.}

Le problème de l'étude en cours se résume dans quelques difficultés linguistiques envisagées par un nombre majoritaire des apprenants(es) de

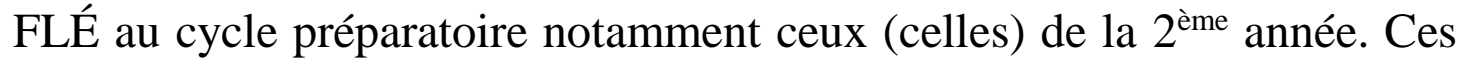
difficultés linguistiques constituent des obstacles devant ces apprenants(es) de ce niveau d'apprentissage pour comprendre, se comprendre, s'exprimer en français. À vrai dire, les difficultés d'apprentissage pour un(e) apprenant(e) de FLÉ sont de plusieurs ordres parmi lesquels se manifestent clairement celles de l'ordre linguistique. Ces genres de difficultés sont dues soit à la faiblesse / à l'imperfection des normes linguistiques (normes grammaticales, structurales, normes morphologiques, normes lexicales et normes sémantiques), soit à la mal 
application de ces normes. Notre problématique s'est posée à travers la question essentielle ci-dessous :-

«Quelle est l'efficacité de l'emploi de l'approche de l'enseignement différencié pour remédier aux difficultés linguistiques en FLÉ chez les élèves du cycle préparatoire ?»

De cette question essentielle, d'autres sous-questions se sont posées telles que:-

- Quelles sont les difficultés linguistiques communes chez les apprenants(es) de FLÉ de la $2^{\text {ème }}$ année préparatoire?

- Quel est le programme d'enseignement vu nécessaire aidant les apprenants(es) de FLÉ, de ce niveau d'apprentissage pour remédier à leurs difficultés linguistiques?

- Quelle est l'efficacité de l'approche de l'enseignement différencié pour remédier aux difficultés linguistiques chez les élèves de ce niveau d'apprentissage ?

\section{Objectifs de l'étude.}

Les objectifs de l'étude actuelle se sont résumés dans :-

- Diagnostiquer les difficultés linguistiques communes chez les apprenants(es) de FLÉ au cycle préparatoire.

- vérifier l'efficacité de l'approche de l'enseignement différencié et de ses stratégies pour améliorer le niveau des apprenants(es) de FLÉ en difficulté linguistique au cycle préparatoire.

\section{Importance de l'étude.}

Il est souhaitable que l'étude actuelle pourrait être profitable pour :-

- Les apprenants(es) de la $2^{\text {ème }}$ année au cycle préparatoire dans la mesure de diagnostiquer et d'améliorer leurs difficultés 
linguistiques (grammaticales, structurales morphologiques, lexicales et sémantiques) à travers un programme d'enseignement élaboré à ce propos.

- Les enseignants(es) de FLÉ de tous les niveaux d'apprentissage soit au cycle préparatoire soit ailleurs dans la mesure d'employer une approche nouvelle, celle de l'enseignement différencié dans le domaine de la didactique de FLÉ. Ils(elles) s'entraînent à bien employer une telle approche pour développer les différentes compétences de leurs apprenants(es) et pour les aider à surmonter leurs lacunes linguistiques en FLÉ.

- Les chercheurs(euses) dans le domaine de la didactique de FLÉ dans la mesure de profiter des outils présentés dans le cadre de l'étude en cours (Typologie de difficultés linguistiques, test de diagnostic de difficultés linguistiques ....etc.).

- Les inspecteurs de FLÉ dans la mesure de renouveler le processus de l'enseignement / apprentissage de FLÉ suivant des stratégies, des techniques, des styles d'enseignement et des approches d'avantgarde.

\section{Hypothèses de l'étude.}

Afin d'atteindre les objectifs de l'étude en cours, on a essayé de savoir si / s' :-

- Il existe une différence statistiquement signifiante entre les moyennes des notes des sujets du groupe de l'étude en ce qui concerne les difficultés linguistiques en entière (grammaticale, structurale, morphologique, lexicale et sémantique) dans les 
résultats de la pré-post application du test de difficultés linguistiques au seuil de (0.01) en faveur de la post application.

- Il existe une différence statistiquement signifiante entre les moyennes des notes des sujets du groupe de l'étude en ce qui concerne les difficultés d'ordre grammatical dans les résultats de la pré-post application du test de difficultés linguistiques au seuil de (0.01) en faveur de la post application.

- Il existe une différence statistiquement signifiante entre les moyennes des notes des sujets du groupe de l'étude en ce qui concerne les difficultés d'ordre structural dans les résultats de la pré-post application du test de difficultés linguistiques au seuil de (0.01) en faveur de la post application.

- Il existe une différence statistiquement signifiante entre les moyennes des notes des sujets du groupe de l'étude en ce qui concerne les difficultés d'ordre morphologique dans les résultats de la pré-post application du test de difficultés linguistiques au seuil de (0.01) en faveur de la post application.

- Il existe une différence statistiquement signifiante entre les moyennes des notes des sujets du groupe de l'étude en ce qui concerne les difficultés d'ordre lexical dans les résultats de la prépost application du test de difficultés linguistiques au seuil de (0.01) en faveur de la post application.

- Il existe une différence statistiquement signifiante entre les moyennes des notes des sujets du groupe de l'étude en ce qui concerne les difficultés d'ordre sémantique dans les résultats de la 
pré-post application du test de difficultés linguistiques au seuil de (0.01) en faveur de la post application.

\section{Délimites de l'étude.}

- Cette étude s'est limitée aux apprenants(es) de FLÉ au niveau préparatoire. Plus précisément, ceux (celles) de la $2^{\text {ème }}$ année de l'école de Khausos officielle des langues au Caire dans la région d'Ain Chams pendant le deuxième semestre de l'année scolaire 2017 / 2018.

- Les difficultés linguistiques se sont limitées à celles des difficultés (grammaticales, structurales, morphologiques, lexicales et sémantiques) de base à ce niveau d'apprentissage.

\section{Outils de l'étude.}

Pour atteindre les objets de l'étude actuelle, on a employé des outils tels que :-

Instruments d'évaluation comme :-

- Test de diagnostic pour déterminer les difficultés linguistiques chez les sujets du groupe de l'étude (ce test est employé comme un test de diagnostic au même titre que test de progrès).

- Typologie de difficultés linguistiques communes chez les apprenants(es) du niveau préparatoire.

* Instruments et matériaux d'enseignement/apprentissage comme :-

- Programme d'enseignement de la linguistique basant sur les apports sous-jacents de l'approche de l'enseignement différencié.

- Guide pédagogique aidant les maîtres(ses) enseignants(es) de FLÉ à bien manipuler le programme de l'enseignement conçu. 


\section{Méthodologie de l'étude.}

En vue d'atteindre les objectifs de l'étude actuelle, le chercheur a suivi les méthodologies ci-dessous :-

- La méthodologie analytique descriptive dans la mesure de la revue de la littérature éducative en ce qui concerne les variables de l'étude actuelle pour présenter un cadre théorique cohérent et relatif à de telles variables.

- La méthodologie expérimentale dans la mesure d'opérer la tentative menée pour surmonter les difficultés linguistiques couramment envisagées à ce niveau d'apprentissage (niveau préparatoire) qui est le cadre de l'étude actuelle.

- La méthodologie statistique qualitative et quantitative dans la mesure de traiter les résultats des sujets du groupe de l'étude et de les interpréter afin de s'assurer de l'efficacité de l'approche de l'enseignement différencié dans le domaine de l'amélioration du niveau linguistique chez les apprenants(es) de FLÉ en difficulté linguistique.

\section{Procédures de l'étude.}

Afin de répondre aux questions posées dans le cadre de l'étude actuelle, on a suivi les démarches ci-dessous :-

Pour répondre à la première question posée, on a adopté les étapes procédurales suivantes :-

- Le chercheur a analysé les études antérieures relatives aux difficultés linguistiques (grammaticales, structurales, morphologiques lexicales et sémantiques) communes chez les apprenants(es) de FLÉ au cycle préparatoire). On a étudié et 
analysé la littérature éducative relative à ce type de difficultés linguistiques déjà mentionnées pour les apprenants(es) de FLÉ pour bien préparer le cadre théorique et pour élaborer un test de diagnostic de telles difficultés.

- Il a présenté ce test de diagnostic des difficultés linguistiques dans sa forme initiale aux spécialistes de la linguistique, aux inspecteurs et aux didacticiens de FLÉ pour le valider.

- Il a préparé le test dans sa forme finale.

- Il a rangé les difficultés linguistiques dans une typologie de difficultés linguistiques communes chez les apprenants(es) de FLÉ suivant le degré de difficultés et on l'a présentée aux spécialistes pour prendre leurs avis.

Pour répondre à la deuxième question posée, on a adopté les étapes procédurales suivantes :-

- Le chercheur a étudié et analysé les études antérieures relatives aux apports sous-jacents de l'approche de l'enseignement différencié et à ses stratégies et techniques employées pour des visées de mettre l'accent sur les rôles de l'enseignant(e), ceux de l'apprenant(e) ainsi les déroulements de l'enseignement / apprentissage adoptés lors de son exploitation dans le cadre de surmonter les difficultés linguistiques envisagées par quelques apprenants(es) de FLÉ au niveau préparatoire en élaborant le programme d'enseignement esquissé dans le cadre de l'étude actuelle et également et pour préparer le cadre théorique.

- Il a également étudié et analysé la littérature éducative relative à l'approche de l'enseignement différencié et à ses stratégies et 
techniques pour l'objet de mettre l'accent sur les rôles de l'enseignant(e), ceux de l'apprenant(e) ainsi les déroulements de l'enseignement / apprentissage adoptés en élaborant le programme d'enseignement esquissé dans le cadre de l'étude actuelle et également et pour préparer le cadre théorique.

- Il a esquissé le programme dans sa forme initiale et l'a présenté aux spécialistes de la linguistique de FLÉ, aux inspecteurs de la langue, et aux didacticiens(iennes) de FLÉ pour le valider en basant sur les résultats de la typologie de difficultés linguistiques.

Pour répondre à la troisième question de l'étude en cours, on a adopté les étapes procédurales suivantes :-

- Le chercheur a commencé notre intervention par la pré-application du test de difficultés linguistiques (test de progrès) pour contrôler le niveau des apprenants / apprenantes de FLÉ avant la tentative.

- Il a travaillé notre programme d'enseignement esquissé à la lueur des apports sous-jacents de l'approche de l'enseignement différencié avec les sujets du groupe de l'étude pour mesurer son efficacité.

- Il a opéré une évaluation continue (évaluation formative) tout au long de notre intervention (on a fait des petits tests de compétences linguistiques sur le contenu de chacune des unités composant le programme esquissé pour s'assurer de façon qualitative du progrès des apprenants(es)).

- Il a fini notre intervention par la post-application du test des difficultés linguistiques (test de progrès) pour évaluer le niveau des apprenants(es) de FLÉ après la tentative. 
- Il a traité les résultats obtenus de façon quantitative pour s'assurer de l'efficacité de l'approche de l'enseignement différencié employée dans le cadre de surmonter les difficultés linguistiques communes chez les sujets du groupe de l'étude.

- On a avancé nos recommandations en proposant des études futures.

\section{Terminologies de l'étude.}

\section{Compétences linguistiques.}

Hodel H. (Hodel H., 2007) a défini le terme de la compétence linguistique comme était les savoirs et savoir-faire relatifs à la sémantique, à la phonologie, à la syntaxe et aux autres dimensions du système linguistique et que l'on a pris comme tel, indépendamment d'aucune valeur sociolinguistique ou autres.

Le ministère de la citoyenneté et de l'immigration canadien a donné une définition du terme (Ministère de la citoyenneté et de l'immigration canadien, 2012) en déterminant que cette compétence linguistique comprend plusieurs concepts et principes parmi lesquels existe la compétence langagière. Celle-ci porte sur la capacité à comprendre et à communiquer des messages de façon efficace et appropriée dans un contexte social précis.

Lüdi G. (Lüdi G., 1997), a défini le terme en déterminant que la compétence linguistique se compose d'autres sous-compétences parmi lesquelles existe celle du lexique composé de listes de mots dans une mémoire lexicale d'une part et de règles lexicales d'autre part. celles-ci (les règles lexicales) servent non seulement à rendre transparentes des unités construites, mais également à produire et à comprendre des unités par un locuteur-auditeur. 


\section{La définition opérationnelle du terme.}

La compétence linguistique comprend plusieurs sous-compétences; celle de la sémantique, celle du lexique, celle de la grammaire et celle de la morphologie dont l'apprenant(e) débutant(e) ou avancé(e) en FLÉ a besoin lors de communiquer correctement oralement ou par écrit avec l'autrui. Ces sous-compétences sont limitées aux compétences structurales, grammaticales, morphologiques, sémantiques et lexicales de base.

\section{Les difficultés linguistiques.}

Nahla Chellouai (Nahla Chellouai, 2013 : 33) a vu que «ces difficultés ont traits aux divers systèmes de règles qui régissent le fonctionnement de la langue en l'occurrence : les règles syntaxiques, morphosyntaxiques, et textuelles (règles de la cohérence) qui aident à la production de différents messages».

\section{La définition opérationnelle du terme.}

Les difficultés linguistiques se sont définies dans l'étude actuelle comme toute lacune ou erreur commises couramment par un nombre énorme des apprenants(es) de FLÉ lors de comprendre ou produire un message langagier (oralement ou par écrit). Ces erreurs sont dans la plupart du temps d'ordre grammatical, structural, morphologique, lexical et sémantique.

\section{L'approche de l'enseignement différencié.}

Jean-Pierre Meyniac (Meyniac J.-P., 2014) a défini la pédagogie différenciée en déterminant que c'est la pédagogie à travers laquelle, l'on peut attribuer à chaque apprenant des tâches correspondantes à ses besoins et à ses possibilités. Selon lui, c'est une différenciation se situant au niveau de chaque apprenant. 


\section{La définition opérationnelle du terme.}

L'approche de l'enseignement différencié s'est vue dans l'étude actuelle dans la mesure que chacun(e) de nos apprenants(es) en difficulté linguistique trouve des possibilités, des activités langagières et des exercices variés leur aidant à remédier à leurs difficultés linguistiques (difficultés grammaticales, structurales, lexicales, sémantiques et morphologiques) communes en employant des stratégies données. Cellesci prennent en considération l'hétérogénéité de leurs différences individuelles ( leurs intérêts, leurs besoins, leurs profils d'apprentissage et leurs niveaux cognitifs) pour comprendre, se faire comprendre pour produire ou bien pour communiquer correctement en FLÉ dans les différentes situations de la vie.

\section{Le cadre théorique.}

Essentiellement parler, l'on ne peut jamais comprendre, se faire comprendre ou produire oralement ou par écrit des messages dans telle ou telle langue vivante étrangère en général et plus particulièrement en français comme langue étrangère sans maîtriser ses normes structurales, grammaticales, morphologiques, lexicales et sémantiques. La langue est bien entendu employée comme un instrument de communication par excellence au niveau de l'oral au même titre qu'au niveau de l'écrit. L'apprenant(e) de FLÉ quel que soit son niveau d'apprentissage forme ses messages oralement et par écrit suivant des normes grammaticales, structurales, morphologiques ...etc., les choisit le lexique convenable suivant des normes lexicales, et les attribue des sens suivant des normes de la sémantique. L'étude en cours a essayé de faire face aux difficultés linguistiques en FLÉ d'une part et d'autre part à envisager la question 
d'hétérogénéité des classes de FLÉ qui est un des caractères de l'ère actuelle. Cette étude a eu un double objectif; le chercheur a pu diagnostiquer les difficultés linguistiques communes chez les sujets du groupe de l'étude et il a élaboré un programme d'enseignement dans des visées à remédier à ces genres de difficultés linguistiques en basant sur les données de l'approche de l'enseignement différencié et ses apports. Afin de bien préparer ce programme, le chercheur a consacré cette partie aux variables de l'étude; les compétences linguistiques et leurs difficultés relatives notamment les difficultés d'ordre grammatical, structural, morphologique, lexical et sémantique et les apports sous-jacents de l'approche de l'enseignement différencié comme arrière-plan méthodologique et didactique.

\section{L'aspect linguistique de FLÉ.}

\section{Rapports notionnels/relationnels de langue/linguistique.}

Les compétences linguistiques sont celles qui constituent des bases structurales englobant des syntagmes, des règles de syntaxe, de phonétique, de morphologie, de lexique et de sémantique que tous les membres d'une même communauté linguistique acquièrent dès les premières années de leur âge. Ces compétences se manifestent inconsciemment lors de la parole au même titre que lors de l'écriture dans toutes les situations d'échanges linguistiques opérées quotidiennement parmi les sujets de cette même communauté.

À ce propos le grand linguiste Ferdinand de Saussure (De Saussure F., 1972 : P. 25) a cité dans son œuvre intitulée «Cours de linguistique générale» que la langue comme : « un produit social [....] est un ensemble de conventions nécessaires adoptées par le corps social». Cette 
confirmation de Ferdinand de Saussure a donné l'impression qu'on peut pas perfectionner les structures de la langue française s'on néglige les conventions de cette langue. Autrement dit, on doit s'intéresser aux aspects essentiels de la linguistique de FLÉ (aspect syntaxique, grammatical, et morphologique et aspect lexical et sémantique).

À la même perspective, Moshé Starets (Starets M., 2000 : P. 94) a confirmé dans son œuvre intitulée "Théories syntaxiques du français contemporain » que la langue est un arbre comportant plusieurs niveaux. Selon lui, le premier niveau constitue le lexique à travers lequel le sujet parlant construit des phrases pour adresser des messages à l'autrui. Plus son lexique est bien choisi, plus ses phrases sont correctes et couvrent le sens voulu par lui. Si l'on désire approfondir ce niveau, il faut bien étudier le lexique de la langue française. Le deuxième niveau constitue la structure des phrases voulues être transmises à l'autrui; c'est la syntaxe. Selon lui, si l'on désire approfondir ce niveau, il faut de façon ou d'une autre avoir cours à bien l'étudier. Celle-ci donne les normes de structurer les phrases du français. Ces phrases composées par le sujet parlant pour être bien prononcées oralement ont une forme phonologique. Selon lui, si l'on désire se communiquer correctement avec l'autrui, comprendre et se faire comprendre oralement, il faut étudier profondément la phonologie, la phonétique et la prosodie. Cette forme phonologique constitue un niveau de la langue française. Les phrases composées par le sujet parlant pour continuer le discours ont une forme logique (C.-à-d., doivent apporter un sens logique). Selon lui ce sens logique est tracé par la coordination des autres niveaux déjà mentionnés. Moshé a présenté une figure éclaircissant les rapports qui existent entre ces différents niveaux linguistiques de la langue en général et de la langue française en 
particulier. Le linguiste veut dire que les compétences linguistiques comprennent plusieurs aspects de la langue en général et la langue française en particulier. Si les apprenants(es) de cette langue cible voudraient la maîtriser, ils (elles) doivent prendre en compte ces différents aspects; Aspect lexical, aspect syntaxique, aspect sémantique, aspect grammatical, aspect morphologique, et aspect phonologique. On a consacré cette étude aux aspects lexical, sémantique, grammatical, structural (syntaxique) et ajoutant également l'aspect morphologique. On n'a pas pu bien sûr approfondir théoriquement ces différents aspects, mais on les a traités de façon simplifiée parce que cette étude est destinée à un public d'apprenants(es) débutants(es) en langue française.

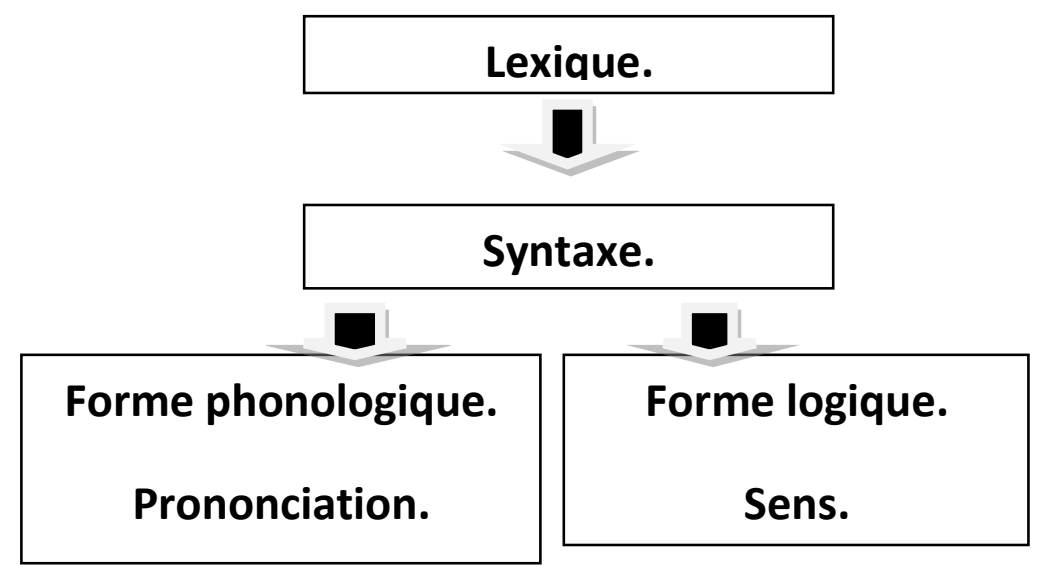

Figure No. ( 1 ).

\section{Arbre linguistique imaginé par Moshé Starets (Starets M., 2000).}

Concernant l'aspect grammatical et structural, il constitue la colonne vertébrale de la langue en général; l'on ne peut jamais apprendre une langue sans qu'il comprenne les normes de la grammaire et de la structure. Albert Hamon (Hamon A., 2007) a confirmé dans l'introduction de son œuvre intitulée «Grammaire et analyse : analyse grammaticale et 
analyse logique » que l'analyse grammaticale constitue la base du processus de la didactique du français au même titre que des autres langues enseignées. La didactique de la grammaire commence automatiquement dès la première séance du processus de l'apprentissage. Ce chercheur a confirmé qu'avec l'analyse grammaticale l'apprenant(e) passe du mot au groupe de mots, du groupe de mots à la proposition, de la proposition à la phrase, de la phrase à l'idée, de l'idée à la pensée, au style, et au plaisir de lire et d'écrire.

La grammaire constitue donc la base élémentaire de toute les autres compétences langagières; l'on ne peut pas imaginer un développement d'autres compétences sans savoir les normes et les conventions grammaticales et structurales. Également le bien savoir de la grammaire aide les apprenants(es) dits débutants(es) en français langue étrangère (FLÉ) à mieux éviter les lacunes envisagées lors de la lecture et de l'écriture. Si cet(te) apprenant(e) a pu éviter ces types de difficultés grammaticales, il(elle) peut se progresser rapidement au sein du processus de l'apprentissage de la langue cible. Maïa Grégoire et Alina Kostucki (Grégoire M., et Kostucki A., 2012) ont affirmé à ce propos que pour les débutants(es) en FLÉ en général, un bon nombre de lacunes grammaticales et structurales sont commises à cause de l'interférence de leur langue maternelle au même titre que les particularités de la langue française dont ils (elles) doivent prendre en considération; selon eux, ces apprenants(es) pensent et forment leurs idées et pensées par la voie de la langue d'origine et s'expriment oralement et par écrit par une langue cible (la langue française). Cela veut dire que si les apprenants(es) désirent maîtriser la langue française pour mieux comprendre, se faire 
comprendre, parler et communiquer à l'oral au même titre qu'à l'écrit, ils (elles) doivent bien pratiquer les normes grammaticales et structurales de FLÉ au même titre que prendre en compte des particularités de cette langue.

Concernant l'aspect lexique et l'aspect sémantique, ils font bien entendu partie intégrale de la compétence linguistique. l'aspect lexical constitue la base langagière de toute acquisition de toute langue étrangère. S'un(e) apprenant(e) de FLÉ voudrait comprendre ou bien produire un message quelconque, il doit premièrement avoir en tête un vocabulaire adéquat aux situations discursives ou écrites, puis savoir les règles lexicales et grammaticales de bien composer des phrases correctes. Francis Grossmann (Grossmann F., 2012) a affirmé à cette perspective que la compétence lexicale ne se réduit pas également à une capacité linguistique d'ordre morphosémantique mais également une compétence dynamique et basée sur une approche intersubjective et évolutive du sens lexical et en même temps sur le rôle actif du contexte dans le processus d'interprétation. D'après le chercheur Grossmann, la compétence lexicale à côté de la compétence sémantique constituent la base linguistique de tout enseignement /apprentissage de FLÉ pour les débutants(es) au même titre que pour les avancés(es).

Concernant l'aspect morphologique, il est à noter que la relation entre l'aspect lexical et l'aspect morphologique sont indissociable parce que l'aspect morphologique constitue la forme des mots et des termes d'une langue. Selon Huot H. (Huot H., 2005) la morphologie constitue non seulement l'étude de la forme des mots dans leurs différents emplois et constructions mais également l'étude de l'interprétation donnée à ces mots 
et termes. L'aspect morphologique est donc complémentaire à celui du lexique dans le cadre de composer le sens voulu derrière l'emploi de ces mots et termes dans le contexte.

Également Manon Alberti et Emma Lavoine (Alberti M., et Lavoine E., 2012) ont défini la morphologie comme l'étude des plus petites unités linguistiques de forme et de sens. Ces deux chercheurs ont mentionné que la morphologie s'intéresse aux morphèmes; ceux-ci sont les petites unités de forme et de sens. Ces chercheurs ont montré que cet aspect morphologique s'intéresse en premier lieu aux structures internes des mots et termes employés soit dans le discours, soit dans la rédaction. D'après eux, l'aspect morphologique constitue l'aspect réglementaire de l'aspect lexical et sémantique pour que le sujet parlant puisse comprendre et produire oralement et par écrit des messages corrects.

Sans entrer dans des détails linguistiques dont on n'a pas actuellement besoin, il est à noter que de maintes chercheurs francophones tels que (Berthes H., 2011), et (Collaud A., 2009) ont éclairci directement ou indirectement la différence existant entre les différents genres de morphèmes notamment les morphèmes lexicaux et les morphèmes grammaticaux en déterminant que les premiers mettent en relief le vocabulaire de la langue française et les deuxièmes ont mis l'accent sur les éléments grammaticaux tels que les pronoms personnels, les conjugaisons des verbes, les articles, les prépositions ainsi les racines des mots et termes et leurs préfixes et suffixes. On peut donc confirmer que les compétences linguistiques qui nous intéressent ici (dans l'étude actuelle) sont sans doute les compétences grammaticales, structurales, sémantiques, lexicales et morphologiques. 


\section{Aperçue historique des compétences linguistiques et leurs difficultés en FLÉ.}

Dans cette partie on a présenté les compétences linguistiques et leurs difficultés du point de vue des méthodologies et des approches essentielles dans le domaine de la didactique de FLÉ telles que la méthodologie traditionnelle (MT), la méthodologie Directe (MD), la méthodologie audio-orale (MAO), la méthodologie audio-visuelle (MAV), l'approche communicative (AC), l'approche actionnelle (AA).

\section{Les compétences linguistiques et leurs difficultés vues par la méthodologie traditionnelle (MT).}

Plusieurs didacticiens(nes), linguistes et chercheurs(euses) tels(telles) que François Golin (Golin F., 1880), Christian Puren (Puren C., 1988), (Puren C., 1995), Germain (Germain C., 1993), Sophie Dufossé Sournin (Dufossé Sournin S., 2007) et Estelle Riquois (Riquois E., 2010) ont théorisé pour la méthodologie traditionnelle et ont déterminé dans leurs écrits les apports sous-jacents de cette méthodologie. La plupart des théoriciens(nes) se sont presque mis(es) d'accord que cette méthodologie est intitulée (La méthodologie traditionnelle, la méthodologie classique, la méthodologie grammaire-traduction ou la méthodologie lexicale). Une telle méthodologie traditionnelle a été employée, selon Christian Puren (Puren C., 1988), dès l'aube du XX ${ }^{\text {ème }}$ Siècle pour enseigner les langues anciennes (Le latin et le grec) qui représentaient à l'époque en France ou ailleurs (dans les autres pays européens) le tiers de l'enseignement au cycle secondaire. Cet enseignement a mis essentiellement l'accent sur la grammaire notamment les règles de grammaire traditionnelle au même 
titre que sur l'explication des textes littéraires. La méthodologie traditionnelle s'est limitée dans la plupart du temps en France à l'enseignement du Latin parce que selon Puren, ce latin était la langue de communication des élites et la langue véhiculaire de toutes les connaissances du temps.

L'objectif majeur de la méthodologie traditionnelle employée avec l'enseignement du latin était de pousser les apprenants(es) de l'époque à bien lire et à bien écrire des textes soutenus (textes littéraires) et également à parler le latin spontanément. La méthodologie traditionnelle s'est intéressée aux compétences linguistiques notamment celles de la grammaire à travers l'application des normes grammaticales, celles du lexique à travers des activités de lecture des textes littéraires, celles de la rédaction de vers à travers la composition littéraire. En bref la méthodologie traditionnelle s'est intéressée à quelques unes des compétences linguistiques.

Les méthodologues partisans à cette méthodologie (MT) ont essayé au fur et à mesure de faire les apprenants(es) éviter les difficultés linguistiques notamment celles de la grammaire. La majorité des méthodologues traditionnels ont expliqué les règles grammaticales au début des cours de la langue et les apprenants(es) les appliquant explicitement à travers les exercices de la traduction (thème et version). La traduction constitue donc le procédé favorable du processus de l'enseignement/apprentissage linguistique. Ces procédures se sont pratiquées dans la méthode de Mauger (1957) cité dans l'étude de Nurten Ozcelik (Ozcelik N., 2012). Il est donc à noter à travers la pratique des 
méthodes traditionnelles que les enseignants(es) évitent les difficultés linguistiques à travers les exercices de «par cœur».

\section{Les compétences linguistiques et leurs difficultés vues par la méthodologie Direct (MD).}

Des didacticiens(nes) nombreux(ses) tels(telles) que Marie-Hélène Clavères (Clavères M.-H., 1985), Christian Puren ( Puren C., 1988) et (Puren C., 1995), Germain (Germain C., 1993), Germain et Séguin (Germain C., et Séguin H., 1998), Sophie Dufossé Sournin (Dufossé Sournin S., 2007) et Estelle Riquois (Riquois E., 2010) ont mentionné que dès la défaite de la France par l'Allemagne et la perte de la région Alsace-Lorraine en 1870, une sorte de renouvèlement au niveau de l'enseignement /apprentissage des langues vivantes étrangères (LVÉ) en général et en particulier au niveau du français langue étrangère (FLÉ) est observable. Ils ont confirmé que les réformateurs de l'enseignement du français ont considéré le modèle allemand comme un modèle exemplaire à suivre; fascinés par ce modèle allemand, ces réformateurs commençaient à s'orienter vers la méthodologie directe exaltant l'aspect oral au dépens de l'aspect écrit de la langue pour des raisons militaires et scolaires en même temps. Ce changement pédagogique a été qualifié par Christian Puren comme « un coup d'état pédagogique de 1902».

Suivant la plupart des didacticiens(nes) déjà mentionnés(es), l'emploi de la méthodologie directe (MD) était dans la mesure de favoriser l'aspect oral au dépens de l'aspect écrit et son emploi s'est limité à des listes de mots (un lexique) mis à la disposition des apprenants(es) afin de les apprendre par cour. Lors de l'enseignement de ce lexique en classe de 
langue, on emploie les gestes, les mimiques, les images et les objets physiques sans aucun passage par l'intermédiaire de la langue maternelle; l'on n'a jamais recours à la traduction vers la langue maternelle. Également on emploie les synonymes et les antonymes en langue française même pour donner le sens des mots. La langue écrite n'est qu'une interprétation scripturée de la langue orale. Cette méthodologie (MD) a mis l'accent sur les compétences morphosyntaxiques de communication quotidienne pour faire passer des messages linguistiquement corrects; tout simplement l'enseignant(e) enseigne des exemples grammaticalement corrects sans entrer dans des détails concernant les règles. Cette méthodologie directe a exalté l'usage du langage dans le discours et non pas de donner des leçons de normes grammaticales comme c'est le cas de la méthodologie traditionnelle. L'essentiel pour une telle méthodologie (la MD) est que ce(s) message(s) passe(nt) correctement dans le cadre du langage parlé (l'aspect oral de la langue française).

Quant aux difficultés linguistiques, aucune pratique linguistique est inscrite dans les écrits méthodologiques et les pratiques adoptant cette méthodologie. Selon Germain et Séguin (Germain C., et Séguin H., 1998), la pratique de l'oral constituait l'objectif majeur d'une telle méthodologie; cette pratique orale précédait toutes les règles linguistiques (grammaticales ou autres). Pour la didactique de la grammaire, les enseignants(es) ont suivi une démarche implicite et inductive. L'enseignant(e) a fait les apprenants(es) répéter de façon mécanique les formes grammaticales comme c'était le cas des méthodes traditionnelles. 
On peut donc déduire que les compétences linguistiques (compétences structurales, grammaticales, morphologiques, lexicales et sémantiques) ne sont pas prises en considération dans les apports sousjacents de la méthodologie directe (MD). Ces types de compétences linguistiques sont médiocrement développées à l'oral mais malheureusement sont totalement négligées à l'écrit parce que l'essentiel pour cette méthodologie est de pousser les apprenants(es) des langues vivantes étrangères en général (LVÉ) et du français langue étrangère (FLÉ) en particulier à parler et à communiquer oralement de façon correcte.

\section{Les compétences linguistiques et leurs difficultés vues par la méthodologie Audio-Orale (MAO) et Audio-visuelle (MAV).}

Des didacticiens(nes), des chercheurs(euses), et des linguistes tels(telles) que Christian Puren ( Puren C., 1988) et (Puren C., 1995), Germain (Germain C., 1993), Germain et Séguin (Germain C., et Séguin H., 1998), Sophie Dufossé Sournin (Dufossé Sournin S., 2007), Overmann (Overmann M., 2009) et Estelle Riquois (Riquois E., 2010) ont théorisé pour les deux méthodologies . La méthodologie audio-orale (MAO) a été largement employée pendant la deuxième guerre mondiale en Amérique pour des buts purement militaires aussi bien que linguistiques; l'objectif principal de cette méthodologie était d'entraîner les militaires à bien se communiquer oralement avec leurs paires parlant d'autres langues que l'anglais et appartenant à d'autres systèmes linguistiques que ceux de la population européenne. D'après les écrits de Christian Puren, cette méthodologie (MAO) a trouvé son support en Amérique grâce aux recherches achevées en psychologie de 
l'apprentissage et en linguistique appliquée de cette époque notamment la linguistique distributionnelle de Bloomfield. Cette linguistique distributionnelle étant un point de référence de l'enseignement /apprentissage de la langue considérant le système linguistique comme un résultat d'analyse syntagmatique et paradigmatique. Or, selon Bloomfield ce sont l'axe paradigmatique et l'axe syntagmatique qu'on peut comprendre lors de manipuler n'importe quel système linguistique. Cette méthodologie audio-orale n'a duré que trois années pendant la guerre mondiale pour répondre rapidement aux nécessités urgentes de la guerre pour faciliter la communication entre les militaires. Cette méthodologie (MAO) a été employée suivant des activités de répétition; la stratégie employée était donc de présenter des exercices de répétition présentés aux apprenants(es).

Concernant les compétences linguistiques et leurs difficultés du point de vue de la méthodologie audio-orale (MAO), l'enseignant(e) présente aux apprenants(es) des listes de vocabulaire (un lexique) pour les apprendre par cœur de façon répétitive. Cette méthodologie était dénommée suivant la méthodologie américaine «la méthodologie situationnelle ». On enseigne chaque langue comme un système dépendant en mettant en considération de façon unique ses composantes linguistiques comme celles de l'aspect phonologique, l'aspect syntaxique, l'aspect morphologique...etc. Or, suivant cette vision, cette méthodologie ne s'est pas occupée de l'aspect sémantique car la signification n'a pas constitué une priorité scolaire. Cette négligence de l'aspect sémantique venait de l'objectif essentiel de l'emploi de la méthodologie dans le processus de l'enseignement /apprentissage. Les compétences sémantique 
étaient donc les seules compétences négligées parmi les autres. Les compétences grammaticales, morphologiques et structurales sont développées par des exercices et des activités de répétitions au niveau de l'oral au dépens de l'écrit. Des exercices structuraux sont donc adoptés en utilisant des techniques modernes à l'époque comme les cassettes pour faire les apprenants(es) les apprendre.

Ces exercices structuraux constituaient le mot clé au sein de la pratique d'une telle méthodologie; c'était à travers ces exercices structuraux que l'enseignant(e) a toujours présenté des structures modèles à les manipuler, donc à les apprendre automatiquement de la part des apprenants(es). Ce mécanisme ont aidé les apprenants(es) à éviter les difficultés linguistiques. Eddy Roulet (Roulet E., 1972 : P. 35) a cité à ce propos que : "la grammaire ne consiste plus, comme dans les grammaires traditionnelles, en un ensemble des règles, mais en une liste de structures». Les exercices consistaient donc à mettre à la disposition des enseignés(es) des structures et des automatismes à les apprendre par cœur. Cuq J.,-P., (Cuq J.-P., 2003) a défini les exercices structuraux comme tout exercice ayant pour but de faire acquérir un comportement linguistique par conditionnement en basant sur un modèle ou un pattern quelconque présenté au début de l'exercice. Cela veut dire que ce type d'exercices aident les apprenants(es) au même titre que les enseignants(es) à éviter toutes les sortes des difficultés linguistiques. Ces exercices structuraux sont donc employés largement avec les méthodologies audio-orales, audio-visuelles et SGAV.

C'est après la seconde guerre mondiale et plus précisément dans les années soixante et soixante-dix du $\mathrm{XX}^{\mathrm{ème}}$ siècle que la méthodologie 
audiovisuelle a trouvé sa naissance grâce aux données des recherches scientifiques dans le domaine de la psychologie structuro-globaliste et de la linguistique distributionnelle de la méthodologie audio-orale d'une part et des supports pédagogiques (enregistrements magnétiques associés à des images fixes) de l'Institut de phonétique de la faculté de lettres à l'université de Zagreb dirigés par Peter Gubérina d'autre part. Cette méthodologie audiovisuelle (MAV) a été influencée par le développement des supports technologiques répandues à l'époque. À ce propos (Germain C., 1993 : P. 155) a cité : «l'apprentissage d'une L2 passe par les sens : l'oreille c'est l'aspect «audio» de la méthode SGAV ${ }^{1}$ et la vue (c'est son aspect «visuel»). L'oreille et l'œil servent de filtres entre les stimuli extérieurs et le cerveau». Germain veut dire que le processus d'enseignement /apprentissage est basé sur l'intégration entre les manuels scolaires d'une part et les supports auditifs et visuels d'autre part. Cette méthodologie audiovisuelle est basée également sur les résultats achevés dans le centre de recherche et d'étude pour la diffusion internationale du français (C. R. É. D. I. F). Ces résultats ont contribué préalablement par des listes du lexique intitulées le français fondamental premier et deuxième degrés (FFI - FFII) et plus tard par ce qu'on a intitulé Niveau-seuil à l'emploi efficace de la méthodologie audiovisuelle.

Selon Peter Gubérina cité dans l'ouvrage de Christian Puren (Puren C., 1988), les usagers de cette méthodologie se sont intéressés aux compétences linguistiques notamment les compétences sémantiques ou bien significatives, les compétences structurales et morphosyntaxiques entrant dans les situations interindividuelles ou bien les compétences qui 
aident à compléter la communication entre les individus parlants. Autrement dit, cette méthodologie audiovisuelle (MAV) a mis en relief toutes les compétences linguistiques qui servent à achever des situations d'échanges orales.

\section{Les compétences linguistiques et leurs difficultés vues par les perspectives communicative et actionnelle.}

De maintes de chercheurs(euses), de didacticiens(nes), de linguistes de FLÉ tels(telles) que Christian Puren (Puren C., 2006), (Puren C., 1995), Evelyne Bérard (Bérard E., 1991), Itziar PLazaola Giger (Plazaola Giger I., 1996), Martinez (Martinez P., 1998), le CECRL ${ }^{2}$ (Conseil de l'Europe, 2001), Emmanuelle Duchiron (Duchiron E., 2003), Sophie Dufossé Sournin (Dufossé Sournin S., 2007), et Estelle Riquois (Riquois E., 2010) ont théorisé en détail et ont présenté les apports sous-jacents des perspectives communicative et actionnelle et ont présenté comment ces approches ont-elles traité les compétences linguistiques en langues vivantes étrangères (LVÉ) en général et en français langue étrangère (FLÉ) en particulier.

Tout d'abord, pour ce qui concerne l'approche communicative, c'est pendant les années soixante-dix du siècle dernier un changement gigantesque au sein de la didactique des langues vivantes étrangères (LVÉ) en général et du français langue étrangère en particulier (FLÉ) s'est passé. C'était la naissance d'une approche nouvelle, l'approche communicative. Cette nouvelle approche a apporté des techniques de classe nouvelles pour le processus de l'enseignement / apprentissage de 
FLÉ; une centration sur l'aspect social de la didactique de la langue s'est observé. Une telle approche a pris l'idée de bien communiquer avec l'autrui comme un point de départ. Suivant un nombre énorme de didacticiens(nes) favorisant l'approche communicative, l'on apprend la langue, c'est pas pour des besoins purement académiques mais des besoins sociaux et notamment des besoins de communication avec les autres sujets de la société en entourage c'est pourquoi la didactique de la langue a commencé traiter des thématiques de la vie courante et quotidienne. Ces thématiques ont été devenues des textes de sports, de voyages, de visites amicales, de faire de courses, de rencontres dans la rue, dans l'ascenseur, dans le métro, ....etc. On a également commencé voir des cours basés sur des situations de la vie quotidienne.

Cette approche communicative a apparu comme une réaction méthodologique et didactique non seulement contre les méthodologies audio-orale et audiovisuelle mais également contre toutes les autres méthodes de l'héritage méthodologique. Elle a mis les besoins linguistiques et langagiers des apprenants(es) en priorité, elle a fait d'eux (d'elles) des gens actifs tout au long du processus de l'enseignement / apprentissage, elle a focalisé pour la première fois sur la fonction et pas sur la forme du langage et de la langue, elle a mis l'accent sur l'interaction verbale et non verbale des apprenants(es) et non pas sur le par cœur du lexique et sur la traduction thème et version comme a fait la majorité des autres méthodologies déjà mentionnées.

Cette approche communicative a été qualifiée suivant Itziar Plazaola Giger (Plazaola Giger I., 1996) comme une approche basée sur la linguistique du langage (de la parole) tandis que les méthodologies 
précédentes sont basées sur la linguistique de la langue. À vrai dire, elle consiste à considérer la langue en général et la langue française en particulier comme un tout englobant les deux aspects (aspect oral et aspect écrit) et les mettant à la disposition de la communication quotidienne. C'est à la moitié des années quatre-vingts du siècle dernier qu'on a commencé à voir en réalité des manuels et des livres préparés suivant les principes de l'approche communicative. Avec cette approche communicative qu'on a commencé à voir largement des manuels et des livres d'enseignement /apprentissage des langues étrangères pour les adultes débutants(es). Autrement dit, les immigrants(es) vers les pays européens ont trouvé facilement des manuels les aidant à mieux s'adapter à la vie quotidienne de leurs nouvelles sociétés.

Concernant le traitement des compétences linguistiques et leurs difficultés, cette approche a considéré la langue comme une unité englobant les différentes compétences linguistiques et les mettant à la disposition des situations de l'enseignement /apprentissage de la langue. Suivant cette approche, on ne donne pas de leçons de grammaire, de structure, de morphologie, du lexique ou bien de la sémantique. L'essentielle est d'enseigner /apprendre la langue comme un tout de façon naturelle dans des situations de la vie courante. Cette approche communicative, comme a confirmé Nurten Ozcelik (Ozcelik N., 2012), a pris en considération que la composante linguistique a compris la connaissance des modèles phonétiques, lexicaux, grammaticaux et textuels semblables à des modèles structuraux suivis dans les méthodologies précédentes. En ce qui concerne les difficultés linguistiques du point de vue de l'approche communicative, on doit se 
conscientiser que les exercices conçus au sein des programmes basés sur une telle approche ne cherchent pas non seulement à surmonter les lacunes, les difficultés et les lapsus linguistiques chez les apprenants(es) de FLÉ mais également à accomplir ou bien à se progresser dans les activités de communication qui constituent l'objectif majeur de l'approche. Cela veut dire que la composante linguistique comme les deux autres composantes (pragmatique et sociolinguistique) sont à la disposition de la communication. En bref, on touche d'après Christian Puren et autres (Puren C., et al., 1998) une sorte de tolérance aux niveaux des erreurs et des lapsus de l'aspect linguistique en général et plus particulièrement de l'aspect grammatical puisque de telles erreurs ne gênent pas la compréhension des messages transmis.

Concernant la perspective actionnelle, des experts(es) et des didacticiens(nes) dans le domaine des langues et des cultures étrangères tels(telles) que Christian Puren (Puren C., 2006), Eva Benetou (Benetou E., 2007), et Nurten Ozcelik (Ozcelik N., 2012) ont montré clairement que la perspective actionnelle a constitué comme une nouvelle idéologie didactique adoptée par les spécialistes en enseignement /apprentissage des langues vivantes étrangères (LVÉ) et leurs propres cultures en général et de langue française étrangère (FLÉ) et sa propre culture en particulier. Le début de cette perspective a commencé dès l'aube du $\mathrm{XXI}^{\mathrm{e}}$ siècle. Cette idéologie s'est adaptée avec les nouvelles orientations du processus de l'enseignement / apprentissage au même titre que la didactique des (LVÉ) et celle du (FLÉ), celles de la pédagogie du projet. Celle-ci a réussi à ajouter une nouvelle dimension à la didactique des langues qui est la dimension sociale afin de donner un sens aux activités langagières 
destinées soit à l'acquisition des compétences de langue (compréhension orale et écrite et production orale et écrite ainsi l'aspect culturel de la langue) soit au développement de telles compétences.

Des chercheurs(euses) nombreuses ont considéré(es) l'approche actionnelle ou bien l'approche par action comme une révolution épistémologique au sein du processus de l'enseignement / apprentissage en général et de la didactique des langues en particulier parce qu'ils / elles ont vu qu'une telle approche est un travail social qui a des finalités sociales. Cette approche est basée sur des activités non filtrées et non fabriquées pour la classe de langue mais sur des activités presque naturelles dans des circonstances brutes dans l'environnement d'enseignement /apprentissage où les apprenants(es) agissent pour achever ces activités comme lors de diriger une entreprise de commerce par exemple, diriger un restaurant, amener les enfants dans une visite à la campagne dans un pays francophone .....etc. Ces activités ne sont pas préparées pour un objectif pédagogique mais pour un objectif social.

Cette approche a été basée sur l'apprentissage actif des apprenants(es); à travers les activités achevées par de tels(les) apprenants(es), ils /elles à leur tour peuvent développer leurs propres compétences langagières. D'après cette approche, les apprenants(es) pratiquent au fur et à mesure des rôles sociaux dans un environnement francophone soit réels soit virtuels pour accomplir les activités proposées. Selon le CÉCRL (Conseil de l'Europe, 2001), cette perspective dite actionnelle a fait les apprenants(es) pratiquer la langue en prenant en considération l'organisation de ses deux facettes; la forme et le sens. Les apprenants(es) des (LVÉ) et du (FLÉ) doivent suivre un trajet qui les conduisent du sens 
vers la forme; lorsqu'ils / elles accomplissent les tâches réelles (un sens), ils / elles sont dus(es) maîtriser les normes linguistiques nécessaires (une forme).

Pour ce qui concerne les difficultés linguistiques, aucune pratique n'est pas touchée au niveau des exercices donnés au sein de l'approche actionnelle. C.,-à-d., on ne consacre pas d' exercices de perfectionnement linguistique mais on retarde cet aspect en exaltant les exercices focalisant sur le sens. Les apprenants(es) en difficulté linguistique sont donc poussés(es) à assister à des programmes remédiaux pour améliorer leurs niveaux linguistiques. Ici les besoins des apprenants(es) ont marqué un impact essentiel pour connaître, voire pour maîtriser cet aspect linguistique. On cherche l'adaptation linguistique de la langue cible selon les situations de la vie quotidienne rencontrées lorsqu'on achève les tâches et cela s'assure lorsque, comme a montré le CÉCRL (Conseil de l'Europe, 2001), l'apprenant(e) se trouve en situation de traduction en L1 ou vers L1, la définition ou l'explication en L2, ou l'utilisation en contexte.

Bref les apprenants(es) de FLÉ peuvent, à travers cette approche dite actionnelle, développer leurs compétences linguistiques (compétences grammaticales, structurales, morphologiques, lexiques, sémantiques et remédier aux difficultés linguistiques par la voie de l'accomplissement des tâches quotidiennes ou par la voie des programmes remédiaux consacrés à ce propos. 


\section{Les rapports approche de l'enseignement} différencié/compétences linguistiques de FLÉ.

Définitions de l'approche ou de la pédagogie différenciée.

On a consacré cette partie du cadre théorique aux apports sousjacents de la pédagogie différenciée en général et plus particulièrement la vision de la tendance francophone du processus de l'enseignement /apprentissage de FLÉ du point de vue de l'approche de l'enseignement différencié mettant ses activités et ses stratégies au service du développement des compétences linguistiques et du traitement des difficultés relatives à ce type de compétences. Tout d'abord, c'est essentiel d'avoir en tête ce qu'on désigne par l'approche de l'enseignement différencié, quelle est sa philosophie de bien gérer les classes hétérogènes de langue, quelles sont ses activités et ses stratégies qu'on peut exploiter afin de développer les compétences linguistiques et en même temps afin de remédier aux difficultés de type linguistique chez les sujets du groupe de l'étude?

Nicole Tardif (Tardif N., 2005) a expliqué ce qu'on désigne par l'approche de l'enseignement différencié en adoptant l'attitude de Camille Deslauriers citée dans le cadre du dossier du travail intitulé «Approche différenciée et Évaluation». Il met en relief que l'approche de l'enseignement différencié n'est qu'une solide pratique du processus de l'enseignement /apprentissage soutenant la progression des apprenants(es) de façon individuelle. Autrement dit, on donne l'occasion à chaque apprenant(e) de développer ses propres compétences linguistiques et paralinguistiques selon ses propres aptitudes, ses rythmes d'apprentissage, son profil d'apprentissage et ses propres capacités. 
Le conseil central de l'enseignement catholique primaire (CCECP, 1988) a clarifié ce qu' on désigne exactement par le concept de la pédagogie différenciée en confirmant qu'on doit être en recherche d'une méditation toujours plus efficace entre l'apprenant(e) et le savoir. Cela signifie que l'enseignant(e) essaie au fur et à mesure de rechercher des outils et des procédures didactiques l'aidant à varier son enseignement / apprentissage.

Stéphanie Descampe et autres (Descampe et al., 2014) ont adopté dans leur livre intitulé «Pratique de la pédagogie différenciée à l'école primaire» le décret de la Communauté française de Belgique disant que la pédagogie différenciée est comme une démarche d'enseignement consistant à varier les méthodes pour prendre en considération l'hétérogénéité des calasses et la diversité des besoins et des styles d'apprentissage des apprenants(es). Ce décret a focalisé sur les classes hétérogènes et sur les besoins et styles diversifiés des individus de la communauté francophone à Belgique et à toutes les autres communautés francophones intéressées à donner l'occasion à ses apprenants(es) de développer leurs propres compétences linguistiques et langagières.

Jean-Michel Zakhatchouk (Zakhartchouk J.-M., 2001) a vu que la pédagogie différenciée est non seulement un moyen de réduire l'effet de l'hétérogénéité constituant un obstacle devant l'égalité de l'apprentissage mais également une source de la richesse et de l'élargissement de cet apprentissage. Ce chercheur a concentré sur le fait que l'enseignant(e) doit s'adapter d'une façon ou d'une autre aux besoins et aux styles de chacun(e) de ses apprenants(es) pour favoriser leurs propres apprentissages. Cela veut dire que les classes hétérogènes deviennent une réalité inévitable dans les établissements scolaires c'est pourquoi les 
enseignants(es) sont invités(es) à varier leurs styles d'enseignement dans les classes de langue.

Dans la même perspective, Léa Beuchat (Beuchat L., 2015) a expliqué le concept de l'approche de l'enseignement différencié en concentrant sur la gestion et la mise en œuvre de l'hétérogénéité au sein des classes de langue au même titre des autres classes à travers des pratiques et des stratégies adaptées à chacun(e) de ces apprenants(es). Ici la chercheuse voit qu' au sein de la classe de langue, les rythmes, les styles d'apprentissage, les stratégies employées soit par les enseignants(es) soit par les apprenants(es) qui se différent selon leurs différences individuelles.

Sans entrer dans des détails monotones de définitions de l'approche de l'enseignement différencié, on peut résumer les points essentiels dont une telle approche a pris en considération selon les écrits des savants :

- La variété des activités et des exercices adoptés et conçus soit par les enseignés(es) eux-elles mêmes soit par les enseignants(es) pour développer ou se faire développer telles ou telles compétences langagières et linguistiques.

- La variété des styles d'apprentissage adoptés par les apprenants(es) au même titre que les styles d'enseignement exploités par les enseignants(es) pour traiter telles ou telles compétences linguistiques suivant bien entendu les genres de telles compétences (grammaticale, structurale, morphologique, lexicale et sémantique).

- La diversification des stratégies d'enseignement et d'apprentissage employées par les enseignants(es) et manipulées par les apprenants(es). 
- La prise en considération des classes hétérogènes en leur exploitant comme une source de richesse et d'élargissement du processus de l'apprentissage et non pas un moyen d'inégalité au sein du développement des compétences langagières et linguistiques de tel(telle) ou tel(telle) apprenant(e).

\section{La pédagogie différenciée face à hétérogénéité.}

On a des motifs solides derrière le choix de l'approche de l'enseignement différencié dans le cadre de l'étude en cours; c'est certain que les classes d'aujourd'hui ne sont plus homogènes soit au niveau intellectuel soit au niveau langagier des apprenants(es); on doit donner l'occasion à chaque apprenant(e) à se progresser vers la maîtrise des compétences linguistiques et langagières. Il faut mentionner que selon Philippe Meirieu (Meirieu P., 1989), pour qu'on fasse un type de différenciation au niveau de la classe (le premier endroit concernant de gérer le processus de l'enseignement/apprentissage), on doit guider les enseignants(es) à être de bon/bonne observateurs /observatrices. Autrement dit, ils / elles doivent suivre leurs apprenants(es) tout le temps durant les activités présentées au sein des séances en classe de langue pour présenter le support aux apprenants(es) en difficulté. Chacun(e) d'apprenants(es) ont le droit de comprendre tel ou tel continu voire de maîtriser toutes les compétences traitées en classe.

Dans la même perspective, Jean-Pierre Megniac (Megniac J.-P., 2014) s'est accordé avec la vision ci-dessus. Il a confirmé qu'on a recours à la pédagogie différenciée pour des raisons d'hétérogénéité des apprenants(es); des raisons cognitives, des raisons culturelles et socioculturelles, et des raisons affectives. Il a assuré que deux raisons 
essentielles sont derrières l'emploi de ce type de pédagogie; premièrement, on aide les apprenants(es) d'acquérir des savoirs et des savoir-faire de base, deuxièmement, on donne l'occasion à chacun(e) des apprenants(es) d'approfondir ses savoirs et ses savoir-apprendre.

Suivant cette vision et en accord avec plusieurs pédagogues et psychologues, on montre que les raisons de type cognitif existent dans les classes parce que les apprenants(es) de l'école préparatoire viennent des environnements d'apprentissage variés. Les raisons de type socioculturel se résument le plus souvent dans les niveaux cultuels et sociaux diversifiés résultant des niveaux financiers. Les raisons de type affectif se limitent le plus fréquemment des différentes attitudes des apprenants(es) envers le processus de l'enseignement /apprentissage en général et envers le processus de la didactique de FLÉ en particulier. Cette vision s'est accordée avec l'étude d'Elena Danescu (Danescu E., 2012).

\section{L'enseignement différencié vs l'enseignement collectif (traditionnel).}

Dans cette partie, les différences essentielles existant entre l'enseignement différencié et l'enseignement traditionnellement pratiqué dans les classes de langue se sont présentées. Le chercheur a recours à plusieurs ressources telles que (Jacqueline C., 2003),(Tomlinson C., 2004), (Tardif N., 2005), (Prupas A., et Patella S., 2007), (Descampe S., et al., 2014), et (Forget A., 2017) pour présenter la comparaison suivante entre l'enseignement différencié et celui collectif (traditionnel). 


\section{L'enseignement Traditionnel.}

Au niveau des finalités et des stratégies d'enseignement /apprentissage.

Les finalités de l'enseignement /apprentissage sont presque collectives et se pratiquent dans une atmosphère collective dirigées par l'enseignant(e) et exécutées par les apprenants(es) de façon collective.

Les stratégies employées au sein de l'enseignement traditionnel sont toujours des stratégies traditionnelles telles que les stratégies de discussion et celles de Questions-Réponses dirigées tout le temps par l'enseignant(e) et exécutées par les apprenants(es) sans prendre en compte les niveaux des apprenants(es) c'est pourquoi on touche une sorte d'échec au sein du développement des compétences langagières et linguistiques chez les apprenants(es).

\section{Au niveau de la gestion du contenu d'apprentissage.}

Ici on a un contenu unique présenté de façon centrale, géré par l'enseignant(e), pratiqué à travers des mêmes activités, et présenté pour tous/toutes les apprenants(es) en même temps. l'enseignement traditionnellement pratiqué considère que les apprenants(es) ont un même niveau c'est pourquoi l'enseignant(e) dirige habituellement le contenu par des mêmes activités et les présente pour tous/ toutes les apprenantes malgré leurs niveaux d'apprentissage et malgré les différences individuelles existant entre eux/elles.

\section{Au niveau des rôles d'apprenants(es).}

Dans ce type d'enseignement, les apprenants(es) ne sont pas responsables de leur apprentissage et sont tout à fait négatifs tout le temps du cours. Ils(elles) reçoivent tout simplement les savoirs, les savoir-faire 
de leurs enseignants(es). Le rôle de l'enseignant(e) dans ce type d'enseignement reste majeur; c'est celui qui conçoit des situations d'apprentissage et également c'est celui qui enseigne tout le temps. les apprenants(es) sont donc des dispensateurs de savoirs c'est pourquoi les acquis d'apprentissage de ce genre d'enseignement restent toujours modestes et les apprenants(es) apprennent par cœur les normes, les listes du vocabulaire, les conjugaisons de verbes ...etc.

\section{Au niveau des rôles d'enseignants(es).}

Dans ce type d'enseignement, le rôle de l'enseignant(e) est majeur; c'est celui qui enseigne, qui conçoit des situations d'enseignement / apprentissage, qui pousse collectivement ses apprenants(es) à répondre aux questions posées, qui donne les savoirs cognitifs à ses apprenants(es), qui développe les savoir-faire méthodologiques chez ses apprenants(es), qui conçoit et dirige les modalités d'évaluation et qui connaît comment apporter remèdes aux difficultés et aux lacunes des apprenants(es) en difficulté linguistique ou d'apprentissage.

\section{Au niveau de la gestion des modalités d'évaluation.}

Ici les enseignants(es) de FLÉ conçoivent seulement les outils et les modalités d'évaluation soit évaluation formative (après chaque partie du manuel scolaire) soit évaluation sommative ou certificative (après avoir terminé le programme scolaire). Ce type d'évaluation est destiné à tous et à toutes les apprenants(es) en même temps. Ils(elles) répondent à leur tour à toutes les questions posées sans aucune prise en considération des différences individuelles existant entre eux (elles) et sans aucune prise en considération de leurs niveaux académiques. Cela veut dire que ce type d'évaluation est destiné à tout le monde malgré leur hétérogénéité qui 
existe de façon naturelle entre les apprenants(es). Cette évaluation est bien sûr inégale.

\section{Quant à l'enseignement Différencié.}

Au niveau des finalités et des stratégies d'enseignement /apprentissage.

Les finalités de l'enseignement /apprentissage sont variées et se pratiquent dans une atmosphère coopérative soit entre l'enseignant(e) et les groupes d'apprenants(es) soit entra groupes (dans les groupes par des stratégies telles que P-C-P (Penser-Comparer-partager) ... etc.

Les stratégies employées dans le cadre de l'enseignement /apprentissage sont dans la plupart du temps des stratégies prenant en considération les niveaux diversifiés des apprenants(es) telles que la stratégie de cercles de discussion et celle de penser-comparer-partager (pc-p) c'est pourquoi on touche une sorte de réussite au sein des acquis d'apprentissage.

\section{Au niveau de la gestion du contenu d'apprentissage.}

Premièrement, le contenu est dans la plupart du temps est varié et plein d'activités diversifiées pour envisager l'hétérogénéité des apprenants(es) au sein des classes de langue. Deuxièmement, on peut également concevoir un seul contenu mais enrichi par des activités variées lors de l'exécution en classe pour envisager les niveaux hétérogènes des apprenants(es).

\section{Au niveau des rôles d'apprenants(es).}

Dans ce genre d'enseignement, les apprenants(es) sont responsables de leur apprentissage et sont eux qui gèrent la majorité de leur travail en classe de langue et le rôle de l'enseignant(e) reste dans le cadre d'un guide 
$\mathrm{du}$ processus. Les apprenants(es) ne sont plus des dispensateurs de savoirs mais ils(elles) deviennent des organisateurs des situations d'apprentissage et également des performants(es) au niveau des savoirfaire. Les apprenants(es) commencent peu à peu grâce à ce type d'enseignement à se diriger vers l'autonomie.

\section{Au niveau des rôles d'enseignants(es).}

Dans ce genre d'enseignement, l'enseignant(e) joue les rôles d'un guide, d'un supporter et d'un conseiller pédagogique. Celui qui présente au fur et à mesure le support en cas de nécessité, qui met les équipements d'apprentissage à la disposition des ses apprenants(es). L'enseignant(e) guide ses apprenants(es) vers l'apprentissage. En bref, l'enseignant(e) en compagnie de ses apprenants(es) se collaborent tout au long du trajet d'enseignement/apprentissage.

\section{Au niveau de la gestion des modalités d'évaluation.}

Ici les apprenants(es) en compagnie de leurs enseignants(es) se collaborent pour préparer les outils et les modalités d'évaluation. Ces outils sont destinés à déterminer leurs propres lacunes au cours de leur trajet d'apprentissage. ils(elles) diagnostiquent leurs lacunes linguistiques, puis ils(elles) dessinent des plans pour traiter ces lacunes linguistiques, ensuite, ils(elles) choisissent les stratégies convenables et ils(elles) déterminent les outils employés pour mesurer leurs propres niveaux avant, pendant et après la tentative afin de savoir le jusqu'à quel point surmontent-ils / elles ces difficultés. On doit prendre en considération que ces apprenants(es) informent leurs enseignants(es) lors d'envisager des difficultés. Dans ce cas les enseignants(es) interviennent pour simplifier les difficultés afin de parvenir à un degré du perfectionnement de la part de leurs apprenants(es). 


\section{Les stratégies de l'approche de l'enseignement différencié.}

Il est à noter que l'approche de l'enseignement différencié peut être appliquée en classe de langue à travers des stratégies d'enseignement /apprentissage dont les plus répandues sont: celle des discussions en groupe et celle de penser-comparer-partager. Ici on a présenté un coup d'œil théorique sans aller plus loin dans des détails méthodologiques. On a exposé de façon pratique comment peut-on employer des telles stratégies dans le cadre de l'étude en cours.

\section{Stratégie de Penser-Pair-Partager, (Penser-Comparer-Partager).}

Cette stratégie est, selon plusieurs ressources parmi lesquelles (Alberta Éducation, 2003), Siara Isaac (Isaac S., 2009) et (Grolleau A.,C., 2010) composée de petits groupes. Elle est employée pour donner l'occasion aux apprenants(es) de partager leurs idées, leurs pensées et leurs solutions proposées à des problèmes. Elle a pour objet de résoudre les problèmes et remédier aux difficultés envisagées par les apprenants(es) de façon collaborative. En d'autres termes, à travers cette stratégie, l'enseignant(e) pose une question quelconque, donne un temps de deux à trois minutes de réflexion et en même temps donne l'occasion à chacun de se discuter à son partenaire pour répondre et enfin chaque équipe de deux partage leur réponse avec la classe toute entière. Cela veut dire donc que cette stratégie a trois étapes : premièrement, on a des équipes de deux apprenants(es) et une question posée à chaque équipe, deuxièmement, un temps de penser ou de réfléchir pour chercher la réponse à la question posée, troisièmement, on commence à partager les réponses avec les membres de la classe. Les activités conçues dans le cadre du programme d'enseignement proposé sont donc destinées à 
employer une telle stratégie. Celle-ci donne l'occasion aux apprenants(es) de FLÉ selon la vision d'Alberta de réfléchir approfondissement à chacune des questions posées, à se discuter avec leurs partenaires (ce qui aident à établir des liens entre eux(elles), à apprendre d'eux(elles) (ce qui les rendent autonomes lors de chercher les réponses), à partager les expériences d'apprentissage avec les autres enseignés(es) de toute la classe (ce qui aident à rendre les apprenants(es) homogènes dans les classes de langue.

Cette stratégie donne donc l'occasion à surmonter les problèmes résultant de l'hétérogénéité existant dans les classes de langues qui est le caractère de nos établissements scolaires à l'ère actuelle. Cette hétérogénéité est à cause de la diversification des systèmes scolaires dans notre société. Cette diversification devient inévitable c'est pourquoi on doit employer des stratégies et des approches d'enseignement prenant en considération les différences individuelles qui existent au sein des classes en général et au sein des classes de langues vivantes étrangères en particulier.

\section{Comment peut-on employer cette stratégie au sein de l'étude actuelle?}

Selon les ressources, cette stratégie se pratique en classe comme suit:-

- On présente la difficulté linguistique sous la forme d'un problème à résoudre (une activité ou un exercice).

- On donne un temps (à peu près 2 minutes) de réflexion (plus il existe un temps de réflexion, plus les réponses des apprenants(es) s'améliorent.

- Chaque apprenant(e) commence à discuter/à comparer sa réponse avec son duo ou son partenaire pour l'améliorer. 
- Enfin on fait appel à quelques pairs ou duos de façon aléatoire pour partager leurs idées avec toute la classe.

- On s'assure à ce moment là que la difficulté linguistique est résolue par tous/toutes les apprenants(es).

- On s'assure donc que les classes linguistiquement hétérogènes deviennent plus en plus homogènes.

\section{Stratégie des discussions en groupe.}

La stratégie des discussions en groupe signifie, du point de vue de plusieurs ressources telles que (Alberta Éducation, 2003), que les apprenants(es) se discutent à travers des groupes pour développer des connaissances sur des sujets déterminés, générer des idées sur des thématiques données afin d'atteindre des objectifs donnés. La plupart des ressources ont montré au fur et à mesure que la réussite du travail exécuté à travers l'emploi de la stratégie donne les enseignés(es) la possibilité de s'exprimer et de présenter de nouvelles idées et de nouvelles informations. Il faut signaler que plus les groupes sont nombreux, plus les idées présentées sont mieux et aident à résoudre la situation problématique. Cette stratégie a plusieurs qualités au sein du travail; elle donne l'occasion à tous les membres de chaque groupe à comprendre et à conscientiser les compétences et les performances demandées, à respecter les besoins de leurs collègues dans et entra les groupes, à prendre part au développement des compétences de leurs pairs, à s'inter comprendre les consignes des questions posées et des situations problématiques, à remédier aux difficultés linguistiques de leurs apprenants(es)...etc.

Cette stratégie joue un rôle primordial dans le cadre de l'approche de l'enseignement différencié car elle peut être employée dans le cadre de 
mettre les membres des classes dans des groupes hétérogènes où ceux qui ont des difficultés linguistiques s'associent avec ceux qui sont favorables en compétences linguistiques. Ce qui permet à tout le monde dans le groupe de profiter des autres.

\section{Les difficultés linguistiques.}

\section{De quoi s'agit-il d'une difficulté linguistique ?}

On désigne par les difficultés linguistiques, les erreurs et les fautes couramment commises par les apprenants(es) de FLÉ lors de parler et d'écrire en français à cause du mal emploi des normes grammaticales, structurales, morphologiques, lexiques et sémantiques. Ces difficultés constituent des obstacles langagiers pour que les messages oraux et écrits ne passent pas ou passent difficilement.

Daniel Nunes Oliveira (Nunes Oliveira D., 2010) a déterminé à travers son étude intitulée «Les difficultés des élèves à communiquer en français à l'oral en classe de $9^{\text {ème }}$ et $10^{\text {ème }}$ au lycée Abilio Duarte de Palmarejo : réalités et perspectives» les différents genres de difficultés en insistant sur celles de la grammaire, celles de la phonétique, celles de la conjugaison, celles de l'orthographe et celles du vocabulaire. Cette étude antérieure avec d'autres telles que celle d'Annie Feyfant et Marie Gaussel (Feyfant A., et Gaussel M., 2007) contribuent à diagnostiquer les problèmes linguistiques essentiels communs pour les apprenants(es).

Il est à noter que les difficultés linguistiques sont dans la plupart du temps derrière les erreurs et les fautes communes dans les productions orale et écrite des apprenants(es) des langues enseignées en général et plus particulièrement dans le (FLÉ). Najib Rabadi et Akarm Odeh (Najib 
Rabadi et Akram Odeh, 2010) ont distingué entre deux genres d'erreurs linguistiques; celles de performances et celles de compétences. Pour les premières, il s'agit des lapsus commis par les apprenants(es) à cause de l'oubli ou de l'inattention. Ici, les apprenants(es) connaissent très bien les règles et ils sont dans la plupart du temps capables de les corriger par eux-mêmes. La plupart des linguistes et des méthodologues les intitulent « des fautes ». pour les deuxièmes, il s'agit des dérivations dues à mal connaissance des règles langagières. Ces types d'erreurs résultent de l'ignorance des normes linguistiques. Les chercheurs ont distingué dans leur étude entre deux genres d'erreurs; des erreurs interlinguales et d'autres intra linguales. Ils ont montré que les erreurs intra linguales sont rares mais on a d'énormes erreurs interlinguales.

Comme un exemple des erreurs et des difficultés dites interlinguales, on envisage celles dues du passage de la langue maternelle vers la langue étrangère et dans notre cas la langue française comme langue étrangère. Dans ce cas, on réfléchit par la langue de départ (l'Arabe) et on s'exprime oralement ou par écrit par la langue d'arrivée (le Français). Houari Meyahi (Houari Meyahi, 2013) a montré qu'il existe des genres de difficultés linguistiques commises dues du passage de la terminologie linguistique française vers celle de l'arabe.

À cet effet Nahla Chelloui (Nahla Chelloui, 2013) a confirmé que les difficultés linguistiques s'attachent aux différents genres de normes de fonctionnement d'une langue donnée (les règles syntaxiques, morphosyntaxiques et textuelles) aidant à produire des messages. Ces difficultés sont d'ordre lexique concernant le mal choix des termes au niveau du contenu, du contexte linguistique, de l'exactitude aux situations 
de la communication, d'ordre morpho syntaxique concernant le mal emploi des règles grammaticales qui régissent l'ordre des mots dans les phrases et des morphèmes grammaticaux qui déterminent le genre, le nombre, le mode, le temps et la personne ....etc.

En bref les difficultés linguistiques constituent des problèmes et des obstacles dus aux lapsus ou aux fautes de performances et aux erreurs de compétences concernant les différents aspects de la langue française (les aspects grammatical, structural, morphologique, morpho syntaxiques, lexique et sémantique). Ces problèmes sont couramment commis par des apprenants(es) étudiant des langues étrangères comme le français en partant des langues maternelles ou d'origine comme l'arabe comme dans le cas de nos apprenants(es).

\section{Les difficultés en compétences linguistiques de FLÉ vues par les linguistes, les approches et les théories linguistiques.}

L'essentiel pour un nombre majoritaire des théories et des approches linguistiques en langue française, est d'accéder au sens au dépens de la forme. Ces théories et approches distinguent ponctuellement entre tout ce qui est linguistique et tout ce qui est extralinguistique. C'est la distinction entre le monde de la langue et le monde du réel. Autrement dit, la distinction entre le monde linguistique et le monde gestuel. Parmi ces théories, la théorie des opérations énonciatives du linguiste Antoine Culioli (Culioli A., 1990) qui a avancé l'hypothèse déjà mentionnée. Cette hypothèse assurant qu'il faut avoir une distinction entre les éléments linguistiques et les éléments extralinguistiques pour accéder facilement au sens.

D'autres linguistes tels(telles) que Danièle Manesse (Manesse D., consulté en 2017) ont une vision inverse. Il voit que l'idée d'exister une 
distinction entre le monde linguistique et le monde réel est en réalité artificielle. Il a confirmé que le monde réel est structuré par le monde linguistique. En d'autres termes, lors de former des messages quelconques soit en oral soit en écrit, on a recours directement au monde de la langue pour les produire.

Des linguistes ont vu que la majorité des difficultés linguistiques résultent de l'impact fort de la langue maternelle sur les processus de l'enseignement /apprentissage des langues étrangères en général et notamment de FLÉ. Ces types de difficultés sont dues aux différences entre la nature de la langue maternelle vis-à-vis à celle de la langue enseignée. Najib Rabadi et Akram Odeh (Najib Rabadi et Akram Odeh, 2010) ont montré dans leur étude qu'en langue arabe, on a deux genres de phrases; la phrase nominale et la phrase verbale mais en français on a uniquement une phrase verbale. Ils ont aussi montré que même la phrase verbale arabe suit un ordre tout à fait différent de celui de la phrase verbale française; en arabe, on dit : «rédige Pierre la lettre» mais en français, on dit : «Pierre rédige la lettre». Lorsque l'apprenant(e) arabe commence à étudier ou à apprendre le français, il commet des erreurs nombreuses à cause de telles interférences linguistiques (au niveau de la syntaxe, de la morphologie, du lexique et de sémantique ....etc.).

Au sein de la sémantique, l'accès au sens en français de la part des autres soit arabes soit d'autres origines qui l'apprennent causent un nombre énorme de difficultés. Le passage d'un système linguistique à l'autre ne constitue pas un simple travail ou bien n'est pas un processus mécanique. La traduction d'une langue maternelle vers la langue cible nécessite des termes et des expressions équivalents pour couvrir exactement le sens. Selon l'étude du linguiste Houari Meyahi (Houari 
Meyahi, 2013), le chercheur a montré que l'on déploie des efforts, au sein de la recherche dans la langue cible pour parvenir à des termes au même titre des structures pour s'approcher le plus possible du sens original. La langue d'après lui n'est qu'une mémoire reflétant le mode de réflexion d'une nation et également qu'un véhicule transportant sa culture. Ce décalage entre deux systèmes linguistiques différents (comme le système linguistique arabe et celui du français) constitue une ressource inévitable de plusieurs difficultés au niveau de l'enseignement / apprentissage de FLÉ.

En bref, les linguistes, les théories linguistiques et les approches didactiques ont confirmé que le fait d'enseigner /d'apprendre le français comme langue étrangère n'est pas un simple processus mais un travail nécessitant des efforts déployés soit de la part des apprenants(es) soit de la part des enseignants(es) pour perfectionner la langue d'arrivée ou bien cible (la langue française). Si vous voulez savoir davantage concernant ce point, vous pouvez consulter des ressources et des études antérieurs telles que (Culioli A., 1990), (Baylon C., et Fabré P., 2003), (Najib Rabadi et Akram Odeh, 2010), (Houari Meyahi, 2013), (Nahla Chelloui, 2013), et (Manesse D., consulté en 2017).

L'étude actuelle a essayé d'avoir comme objectifs les difficultés linguistiques communes chez les apprenants(es) de FLÉ. Ces difficultés se sont résumées des compétences grammaticales, structurales, morphologiques, lexicales et sémantiques de base. Elle a visé également à présenter un programme d'enseignement à la lueur de l'approche de l'enseignement différencié et ses mécanismes du travail afin d'aider ces genres d'apprenants(es) notamment les sujets du groupe de l'étude à remédier à leurs propres difficultés linguistiques. Afin d'atteindre ces 
objectifs, on a élaboré des outils et des matériaux d'enseignement/ apprentissage et d'évaluation et ces outils font partie intégrale des pages et des lignes ci-dessous.

\section{Étude du champs.}

À travers cette étude, le chercheur a visé à rendre les apprenants(es) de FLÉ au niveau préparatoire capables de remédier à leurs propres difficultés linguistiques déjà mentionnées pour comprendre et produire des messages correctement et spontanément dans les différentes situations; ils(elles) deviennent capables de composer des phrases grammaticalement, morphologiquement, structuralement, lexicalement et sémantiquement correctes. il a conçu un programme d'enseignement basé sur les apports sous-jacents de l'approche de l'enseignement différencié avec d'autres outils et matériaux de recherche vus nécessaires à ce propos. il a choisi les apprenants(es) de FLÉ de ce niveau d'apprentissage car ils (elles) sont hétérogènes au niveau des compétences linguistiques au même titre que les autres compétences. En d'autres termes ces genres d'apprenants(es) sont diversifiés du point de vue de leurs niveaux linguistiques, académiques, leurs intérêts d'apprentissage, leurs profils d'apprentissage et leurs aptitudes cognitives et plus particulièrement leurs savoirs et savoir-faire en FLÉ; quelques uns(es) ont un niveau débutant, d'autres ont un niveau avancé, et d'autres ont un niveau linguistique élevé du raison qu'ils(elles) ont déjà étudié le français. Or les classes sont normalement hétérogènes du point de vue des savoirs linguistiques ou extralinguistiques. On part du principe d'égalité qui considère que tous et toutes les apprenants(es) ont le droit de perfectionner les différentes compétences de la langue et notamment les compétences linguistiques. 
Cette partie est donc consacrée à valider les matériaux et les outils de l'étude actuelle qui sont :-

- Le test de difficultés linguistiques destiné aux apprenants(es) de FLÉ de ce niveau d'apprentissage (ce test est employé comme test de diagnostic et test de progrès).

- La typologie de difficultés linguistiques communes chez les apprenants(es) de FLÉ de ce niveau d'apprentissage.

- Le programme d'enseignement élaboré à des visées d'aider ces apprenants(es) de FLÉ de ce niveau d'apprentissage à remédier à leurs difficultés linguistiques.

- Le guide pédagogique aidant les maîtres(ses)-enseignants(es) de FLÉ à appliquer le programme d'enseignement proposé.

\section{Concernant le test de difficultés linguistiques pour les apprenants(es)} de FLÉ au cycle préparatoire.

Ce test a eu pour objectif de déterminer le niveau des apprenants(es) de FLÉ au cycle préparatoire dans les compétences linguistiques et de déterminer et diagnostiquer leurs difficultés linguistiques.( $C f$. Annexe No.1). Ce test a compris (6) questions essentielles dont chacune s'est composée de (5) items couvrant les différents aspects des compétences linguistiques qui font des difficultés pour les apprenants(es). Les questions ont été de type objectif et direct; les apprenants(es) ont été demandés(es) à compléter des phrases, de former des situations, de dire autrement...etc. Ce test a été évalué par un nombre de spécialistes en linguistique, en méthodologies et en didactique de FLÉ pour s'assurer qu'il est convenable au niveau des apprenants(es). Ce test a mis en relief 
les difficultés communes chez les apprenants(es) de FLÉ de ce niveau d'apprentissage telles que (l'emploi des connecteurs au sein des phrases simples, l'emploi des connecteurs au sein des phrases composées, l'art de bien conjuguer les verbes couramment employés dans les situations de la vie courante aux modes et aux temps convenables, l'emploi des morphèmes lexicaux et grammaticaux de base, l'emploi correct des adjectifs, des adverbes et des prépositions de base au sein des phrases françaises, le choix du vocabulaire adéquat pour s'exprimer correctement en français facile, l'emploi des synonymes et des antonymes au sein des phrases composées pour accéder simplement au sens).

Le chercheur a fait une échelle descriptive de ce test pour s'assurer qu'il a couvert toutes les difficultés linguistiques diagnostiquées et mesurées :-

Tableau no. ( 1 ).

Échelle descriptive des items du test.

\begin{tabular}{|l|l|l|l|l|}
\hline No. & $\begin{array}{l}\text { Genre de difficultés } \\
\text { linguistiques } \\
\text { diagnostiquées et } \\
\text { mesurées }\end{array}$ & $\begin{array}{l}\text { Les items } \\
\text { correspondants. }\end{array}$ & $\begin{array}{l}\text { Nombre } \\
\text { d'items. }\end{array}$ & Pourcentage. \\
\hline 1. & $\begin{array}{l}\text { Difficultés } \\
\text { grammaticales. }\end{array}$ & $\begin{array}{l}\text { I.d. / I.e. / II.a. / } \\
\text { II.b. / II.c./ II.d./ } \\
\text { II.e./ }\end{array}$ & 7 & $23.33 \%$ \\
\hline 2. & Difficultés & V.a./ V.b./ V.c./ & 5 & $16.66 \%$ \\
\hline
\end{tabular}




\begin{tabular}{|c|c|c|c|c|}
\hline & structurales. & V.d./ V.e./ & & \\
\hline 3. & $\begin{array}{l}\text { Difficultés } \\
\text { morphologiques. }\end{array}$ & $\begin{array}{ll}\text { I.a./ I.b./ } & \text { I.c. I } \\
\text { VI.d./ } & \text { VI.e./ } \\
\text { VI.a./ } & \text { VI.b./ } \\
\text { VI.c./ } & \end{array}$ & 8 & $26.66 \%$ \\
\hline 4. & Difficultés lexicales. & $\begin{array}{ll}\text { III.a./ } & \text { III.b./ } \\
\text { III.c./ } & \text { III.d./ } \\
\text { III.e./ } & \end{array}$ & 5 & $16.66 \%$ \\
\hline 5. & $\begin{array}{l}\text { Difficultés } \\
\text { sémantiques. }\end{array}$ & $\begin{array}{ll}\text { IV.a./ } & \text { IV.b./ } \\
\text { IV.c./ } & \text { IV.d./ } \\
\text { IV.e./ } & \end{array}$ & 5 & $16.66 \%$ \\
\hline 6. & Total des Difficultés. & - & 30 & $100 \%$ \\
\hline
\end{tabular}

Le chercheur a opéré une application pilote du test sur un groupe d'apprenants(es) en éliminant ceux et celles du groupe de l'étude pour le valider. il a employé les techniques statistiques convenables pour calculer la durée du test, sa fidélité, sa validité et son indice de facilité / difficulté et ses critères de notation. (Cf. Annexe No. 5). il a trouvé que la durée du test $=90$ minutes +5 minutes pour expliquer ses consignes, la fidélité $=$ 0.87 , la validité $=0.932$ et l'indice de facilité / difficulté est entre 0.29 et 0.77. Ces valeurs ont été suffisantes pour appliquer ce test dans le cadre de notre intervention expérimentale. Après avoir terminé toutes ces étapes procédurales, il s'est assuré que le test devient valable d'être employé comme outil de diagnostic et d'évaluation dans le cadre de l'étude en cours. 
Critères de notation du test.

Ce test a compris (30) items et on a consacré (2) points à chaque item. Or la note totale du test $=(60)$ points.

Concernant la typologie de difficultés en compétences linguistiques chez les apprenants(es) de FLÉ au cycle préparatoire.

Cette typologie de difficultés a visé essentiellement à délimiter le degré de fréquence des lacunes, des lapsus et des difficultés linguistiques chez les apprenants(es) de FLÉ visés(es). Ces difficultés se sont résumées en (5) genres essentiels dont (3) genres sont d'ordre grammatical, (1) genre est d'ordre structural, (2) genres sont d'ordre morphologique et (2) genres sont d'ordre sémantique et (2) genres sont d'ordre lexique. On a présenté cette typologie dans sa forme initiale à un nombre de spécialistes en linguistique de français, en méthodologies de langues, en didactique de FLÉ. Les membres du jury l'ont appréciée et ont suggéré quelques modifications dont le chercheur a opéré. La typologie dans sa forme finale (Cf. Annexe No. 2) a constitué un point de départ et une étape procédurale importante et nécessaire pour élaborer le programme proposé en déterminant ses objectifs, ses composantes essentielles et ses modalités d'évaluation. Cette typologie a été présentée après avoir appliqué le test de diagnostic (test de difficultés linguistiques élaboré et manipulé par le chercheur) sur un groupe d'apprenants(es) choisis(es) aléatoirement parmi les ceux(celles) du niveau préparatoire. Le chercheur a considéré que chaque apprenant(e) a obtenu $40 \%$ au maximum dans le test en entier ou dans chacune des difficultés contenues dans le test a été en difficulté linguistique et a eu besoin de l'intervention par le programme remédial 
proposé. Cette typologie a essayé également de focaliser sur les difficultés linguistiques découvertes et déterminées par le test de diagnostic. Ces difficultés se sont résumées dans les difficultés suivantes: les difficultés au sein de (l'emploi des connecteurs au sein des phrases simples, l'emploi des connecteurs au sein des phrases composées, l'art de bien conjuguer les verbes couramment employés dans des situations de la vie courante, réguliers ou irréguliers, aux modes et aux temps convenables, l'emploi correct des adjectifs, des adverbes et des prépositions de base au sein des phrases françaises, l'emploi des morphèmes lexicaux et grammaticaux de base, le choix du lexique adéquat pour s'exprimer correctement en français facile dans les situations de la vie, l'emploi des synonymes et des antonymes au sein des phrases composées pour accéder simplement au sens). Cette typologie est employée dans le cadre de l'étude actuelle comme un point de départ pour premièrement catégoriser ces difficultés suivant le degré de fréquence et pour mettre en relief les difficultés linguistiques à remédier. Cette typologie a constitué donc une des procédures essentielles de l'étude actuelle.

Après avoir appliqué le test de diagnostic, on a obtenu la typologie de fréquence des difficultés linguistiques comme suit 
Tableau no. ( 2 ).

Degré de fréquence des difficultés linguistiques chez les apprenants(es) du niveau préparatoire.

\begin{tabular}{|c|c|c|}
\hline No. & $\begin{array}{l}\text { Genres de difficultés } \\
\text { linguistiques diagnostiquées. }\end{array}$ & $\begin{array}{l}\text { Degré de fréquence des } \\
\text { difficultés linguistiques } \\
\text { (pourcentage suivant les sujets } \\
\text { du groupe de l'étude). }\end{array}$ \\
\hline 1- & \begin{tabular}{lrr} 
Difficultés & \multicolumn{2}{r}{ d'ordre } \\
grammatical et structural \\
communes chez les \\
apprenants(es) de FLÉ.
\end{tabular} & $\begin{array}{l}\text { Environ de } 60 \% \text { (faibles). } \\
\text { c.-à-d., } 40 \% \text { ont correctement } \\
\text { répondu aux items de ce genre } \\
\text { de difficulté. }\end{array}$ \\
\hline 2- & $\begin{array}{lrr}\begin{array}{l}\text { Difficultés d'ordre } \\
\text { communes lexical } \\
\text { chez }\end{array} & \text { les } \\
\text { apprenants(es) de FLÉ. } & \end{array}$ & $\begin{array}{l}\text { Environ de } 61 \% \text { (faibles). } \\
\text { c.-à-d., } 39 \% \text { ont correctement } \\
\text { répondu aux items de ce genre } \\
\text { de difficulté. }\end{array}$ \\
\hline 3- & $\begin{array}{l}\text { Difficultés } \\
\text { morphologique } \begin{array}{r}\text { d'ordre } \\
\text { communes }\end{array} \\
\text { chez les apprenants(es) de } \\
\text { FLÉ. }\end{array}$ & $\begin{array}{l}\text { Environ de } 69 \% \text { (faibles). } \\
\text { c.-à-d., } 31 \% \text { ont correctement } \\
\text { répondu aux items de ce genre } \\
\text { de difficulté. }\end{array}$ \\
\hline 4- & $\begin{array}{l}\text { Difficultés d'ordre } \\
\text { sémantique communes chez } \\
\text { les apprenants(es) de FLÉ. }\end{array}$ & $\begin{array}{l}\text { Environ de } 71 \% \text { (faibles). } \\
\text { c.-à-d., } 29 \% \text { ont correctement } \\
\text { répondu aux items de ce genre } \\
\text { de difficulté. }\end{array}$ \\
\hline
\end{tabular}

Concernant le programme proposé pour remédier aux difficultés linguistiques communes chez les apprenants(es) de FLÉ au cycle préparatoire. 
Ce programme a eu pour objectif de remédier aux difficultés linguistiques couramment envisagées par les apprenants(es) de FLÉ du niveau préparatoire à la lueur des apports sous-jacents de l'approche de l'enseignement différencié. Cette approche emploie toujours des stratégies convenables servant à dissocier les difficultés linguistiques en compétences simples et faciles à développer chez les sujets du groupe de l'étude. Ces difficultés se sont présentées sous la forme des compétences à développer, ensuite des objectifs à atteindre. Les objectifs essentiels de ce programme sont donc:-

- L'emploi correct des connecteurs au sein des phrases simples;

- L'emploi correct des connecteurs au sein des phrases composées;

- L'art de bien conjuguer les verbes couramment employés réguliers ou irréguliers aux modes et aux temps convenables;

- L'emploi correct des adjectifs, des adverbes composés et des prépositions au sein des phrases françaises;

- L'emploi correct des morphèmes lexicaux de base;

- L'emploi correct des morphèmes grammaticaux de base;

- Le choix exact du lexique adéquat pour s'exprimer correctement en français facile dans les différentes situations;

- L'emploi correct des termes, des synonymes et des antonymes au sein des phrases composées pour accéder simplement au sens.

On a employé des stratégies variées au sein du programme élaboré. Ces stratégies sont dans la plupart du temps :- 
- Stratégie de (PPP) ou de (PCP); Penser-Pair-Partager/ PenserComparer-Partager.

- Stratégie des discussions en groupe.

Et d'autres stratégies telles que SVA (tableaux de SVA) et RM (remue-méninges) et celle de la discussion. On a employé au sein du programme des techniques d'enseignement telles qu'un laptop, des CDs, de l'Internet, des feutres de couleurs variées, des fichiers, des livres de grammaire, de structure, et de morphologie de base, des dictionnaires du lexique et de la sémantique de base. L'évaluation au sein du programme élaboré s'est opérée de façon formative à travers les petits tests présentés pendant la tentative pour mesurer le progrès des apprenants(es), et de façon sommative à travers le test de progrès présenté au début et à la fin du programme pour mesurer le niveau des apprenants(es) avant et après la tentative pour s'assurer de l'efficacité du programme et l'efficacité de l'approche de l'enseignement différencié. Pour voir les détails de ce programme, vous êtes priés(es) de consulter l'annexe relatif (Cf., annexe No. 3).

\section{Le déroulement du travail dans le programme élaboré.}

Le programme s'est composé de ( 3 ) unités essentielles + unité ( 0 ) et chaque unité a compris un nombre d'activités traitant les difficultés contenues dans les objectifs de l'unité.

Chaque activité dans le programme est conçue selon l'approche de l'enseignement différencié et ses stratégies suivant des étapes successives comme suit :- 


\section{- L'étape de PENSER.}

À travers cette étape, on a présenté la difficulté linguistique sous la forme d'un problème à résoudre aux apprenants(es) de FLÉ et plus précisément un exercice ou une activité à faire par les apprenants(es) de façon personnelle. L'enseignant(e) a consacré un temps de réflexion (à peu près 2 ou 3 minutes) pour avoir en tête des idées comme des solutions logiques ou des réponses probables.les apprenants(es) ont du avoir en tête que la qualité des réponses et des idées proposées comme solutions s'améliorent suivant la durée consacrée à la réflexion et suivant les discussions menées entre eux/elles. ceux / celles-ci se sont mis à réfléchir et à avoir des idées concernant le problème ou la difficulté linguistique donnée.

\section{- L'étape de COMPARER.}

À travers cette étape, les apprenants(es) ou les participants(es) aux séances consacrées au travail commencent à former des pairs ou des duos et discutent leurs idées et leurs solutions avec leurs pairs. Cette étape a donné l'occasion aux apprenants(es) participants(es) d'améliorer leurs idées, leurs réponses, et leurs solutions aux problèmes ou aux difficultés linguistiques. Chacun peut profiter d'expériences et des savoirs antérieurs de son pair ou de son duo.

\section{- L'étape de PARTAGER.}

À travers cette étape, les enseignants(es) font appel aléatoirement à quelques uns(es) parmi ces pairs ou duos pour partager leurs idées, leurs réponses et leurs solutions avec toute la classe. Dans ce cas les 
apprenants(es) peuvent s'assurer de l'exactitude de leurs réponses. Ces étapes procédurales ont constitué les bases essentielles des déroulements pratiqués tout au long des séances consacrées aux leçons composant le programme proposé. Il est à noter que le chercheur a dessiné des versions des fichiers employés pour répondre à chaque activité pendant les séances du programme proposé suivant cette stratégie et en même temps suivant le type de difficulté (Cf. Les annexes du programme).

\section{Le choix du groupe de l'étude et l'exécution de la tentative.}

Notre groupe de l'étude a été choisi parmi les apprenants(es) de la $2^{\text {ème }}$ année au cycle préparatoire qui ont étudié le français comme langue étrangère (FLÉ). Ce groupe a compris (33) apprenants(es) de (FLÉ) dans l'école de Khausos officielle des langues au Caire dans la région d'Ain Chams. Le groupe a compris (36) apprenants(es) au début de la tentative mais (3) sont été éliminés(es) parce qu'ils(elles) se sont absentés(es) 2 reprises successives. Ce programme a été travaillé pendant 2 mois du travail sérieux; la séance était 90 minutes hors de l'emploi du temps de l'école et on a opéré 2 séances par semaine, donc 8 séances par mois (16 séances consacrées à la tentative). Or, notre programme a duré 24 heures du travail sérieux.

Concernant le guide pédagogique employé par les maîtres(ses) enseignants(es) pour bien manipuler le programme proposé.

Le guide pédagogique préparé dans le cadre de l'étude actuelle est destiné à guider les maîtres(ses)-enseignants(es) de FLÉ au cycle préparatoire à bien employer le programme élaboré à la lueur des apports sous-jacents de l'approche de l'enseignement différencié et ses 
mécanismes du travail pour remédier aux difficultés linguistiques couramment envisagées par les apprenants(es) de FLÉ de ce niveau d'apprentissage. Il a joué le rôle de faire ces maîtres(ses)-enseignants(es) au même titre les apprenants(es) conscientiser des types de difficultés linguistiques, les mécanismes de comment les affronter, les stratégies d'enseignement /apprentissage employées pour comment leur apporter des solutions et les étapes employées opérationnellement pour faire face à chaque type de ces difficultés linguistiques. Ce guide dans sa copie initiale à été présentée à un nombre de spécialistes (des encadreurs de méthodologies de LVÉ, des encadreurs de la didactique de FLÉ, et des encadreurs de linguistique de FLÉ). Ces spécialistes ont inscrit des remarques dont on a pris en compte dans la copie finale $(C f$., annexe no. 4).

Ce guide pédagogique contient une introduction mettant en relief les apports sous-jacents de l'approche de l'enseignement différencié, les objectifs généraux des unités du programme élaboré, leurs objectifs opérationnels, leurs stratégies d'enseignement/apprentissage, leurs techniques d'enseignement /apprentissage employés pendant le travail, leurs matériaux employés, et les types de difficultés traitées dans chaque unité de ce programme.

\section{Étude expérimentale.}

Cette étude a essayé de proposer un programme à la lueur des apports sous-jacents de l'approche de l'enseignement différencié pour améliorer le niveau des apprenants(es) de FLÉ au niveau préparatoire et les aider à remédier à leurs difficultés linguistiques. Après avoir appliqué le test de 
difficultés linguistiques en question avant la tentative et après avoir fini notre intervention par le programme élaboré dans la visée d'améliorer le niveau des apprenants(es) de FLÉ en difficulté linguistique, et après avoir appliqué le test de difficultés après la tentative, le chercheur a traité les notes brutes obtenues et il les a analysées par les techniques statistiques convenables à travers le bagage statistique de (SPSS). Les résultats de l'étude se sont résumés dans :-

\section{Les résultats de l'étude.}

\section{Pour la vérification de la première hypothèse.}

Cette première hypothèse est : «Il existe une différence statistiquement signifiante entre les moyennes des notes des sujets du groupe de l'étude en ce qui concerne les difficultés linguistiques en entière (Difficultés grammaticale, structurale, morphologique, lexique et sémantique) dans les résultats de la pré-post application du test des difficultés linguistiques au seuil de (0.01) en faveur de la post application». Pour vérifier cette hypothèse, le chercheur a traité les notes brutes de l'application du test de difficultés linguistiques avant et après la tentative par la technique statistique intitulée «SPSS» et on a obtenu les valeurs contenues dans le tableau ci-dessous :- 
Tableau no. ( 3 ).

Résultats relatifs au test des difficultés linguistiques (test de diagnostic) en entier.

\begin{tabular}{|c|c|c|c|c|c|c|c|}
\hline $\begin{array}{l}\text { Difficulté } \\
\text { mesurée }\end{array}$ & $\begin{array}{l}\text { Application } \\
\text { du test. }\end{array}$ & $\begin{array}{l}\text { Nombre } \\
\mathrm{du} \\
\text { groupe. }\end{array}$ & $\begin{array}{c}\text { Moyenne } \\
\text { arithmétique. } \\
\text { (M.) }\end{array}$ & $\begin{array}{l}\text { Écart } \\
\text { type. }\end{array}$ & $\begin{array}{l}\text { Valeur } \\
\text { de T. } \\
\text { calculée. }\end{array}$ & $\begin{array}{c}\text { Valeur } \\
\text { de/d' } \\
\text { ETA }^{2} \\
(\eta)\end{array}$ & $\begin{array}{l}\text { S. } \\
\text { ou } \\
\text { NS. }\end{array}$ \\
\hline \multirow{2}{*}{$\begin{array}{l}\text { Les items } \\
\text { du test en } \\
\text { entier. }\end{array}$} & Pré - test. & \multirow[b]{2}{*}{33} & 21.5758 & 3.23101 & \multirow[b]{2}{*}{43.941} & \multirow[b]{2}{*}{0.98} & \multirow{2}{*}{$\begin{array}{c}\text { S. } \\
\text { au } \\
\text { seuil } \\
\text { de } \\
0.01\end{array}$} \\
\hline & Post - test. & & 48.0000 & 5.47723 & & & \\
\hline
\end{tabular}

\section{Commentaire du tableau.}

Le tableau no. ( 3 ) montre le nombre du groupe de l'étude ( 33 ), la moyenne arithmétique pour la pré-application du test de difficultés linguistiques $=(21.5758)$, celle pour la post-application du test de difficultés linguistiques $=(48.0000)$, l'Écart type pour la pré-application $\mathrm{du}$ test $=(3.23101)$, celui pour la post-application du test $=(5.47723)$, la valeur de $« \mathrm{~T} »$ calculée $=(43.941)$, la valeur d'ETA ${ }^{2}=(0.98)$, et l'indice de signifiance $($ S. ou NS.) $=$ S. au seuil de $(0.01)$.

Ces valeurs ont assuré qu'il existe une différence signifiante au seuil de (0.01) entre les moyenne des notes des apprenants(es) du groupe de l'étude. Ce qui a assuré l'efficacité du programme proposé pour surmonter les difficultés linguistiques envisagées par les sujets du groupe de l'étude. Or, la première hypothèse de l'étude actuelle a été vérifiée. Cela signifie que le traitement des différentes difficultés linguistiques contenues dans le test de diagnostic à travers les apports de l'approche de l'enseignement 
différencié était efficace dans le domaine de faire face à ces mêmes difficultés en entière. Cela s'est apparu clairement à travers le schéma statistique individuel ci-dessus où on a remarqué une comparaison entre les niveaux des sujets du groupe de l'étude avant et après la tentative.

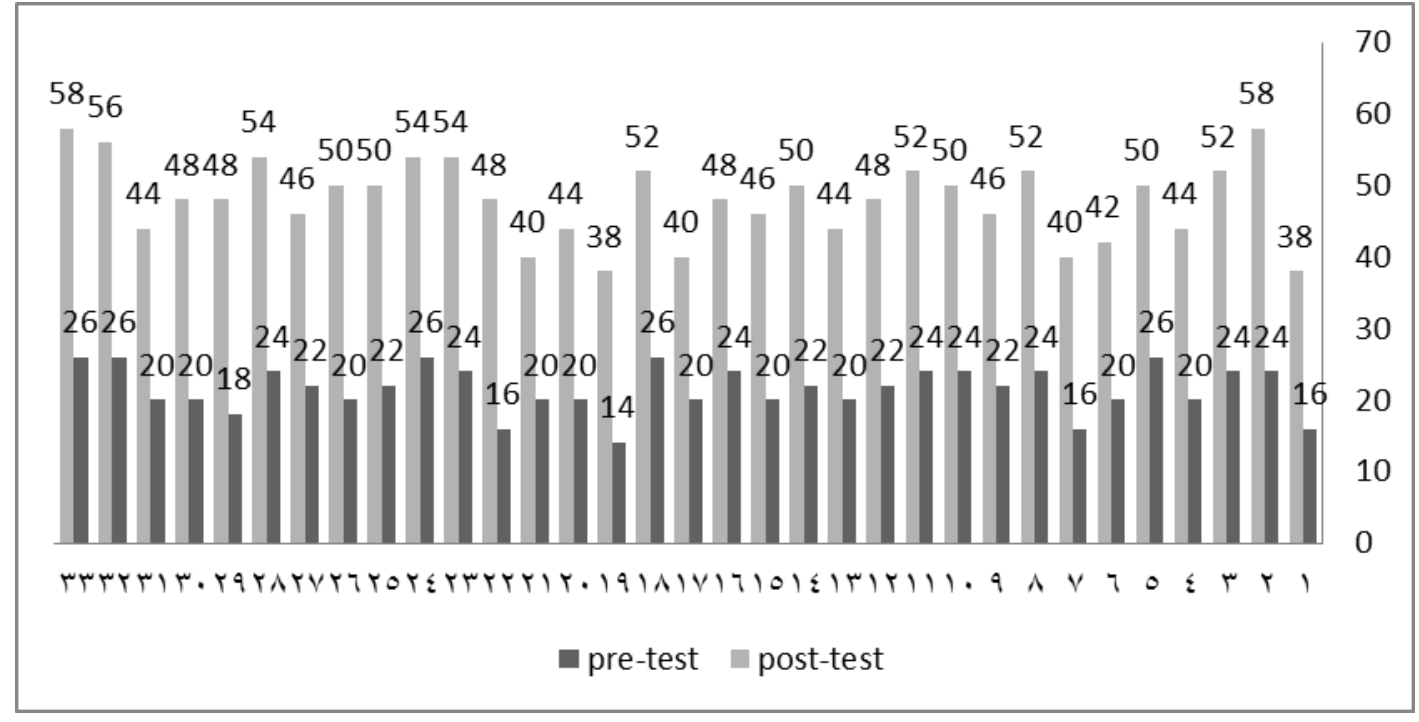

Analyse qualificative du résultat des apprenants(es) pour les difficultés linguistiques en général.

Le chercheur a observé, à travers le schéma ci-dessus, qu'avant la tentative la majorité massive des points des sujets du groupe étaient entre (14 et 26 points) dans les items de difficultés linguistiques toutes entières. Après avoir terminé la tentative, ces points sont clairement améliorés; les points des sujets sont devenus entre (40 et 58 points). Le chercheur a noté que :-

- $\quad 12 \%$ du groupe de l'étude $(\mathrm{n}=4)$ ont obtenu $\geq 16$ points sur 60 points (note totale) au début de la tentative mais au contraire après avoir terminé la tentative, $30 \%$ du groupe de l'étude $(\mathrm{n}=$ 10) ont obtenu $\geq 44$ points sur 60 . Cela veut dire que $10 \% \mathrm{du}$ groupe de l'étude ont obtenu ( $74 \%$ de la note totale). Ce progrès 
considérable est du à l'application du programme élaboré, ce qui a assuré l'efficacité de l'approche de l'enseignement différencié.

- $88 \%$ du groupe de l'étude $(n=29)$ ont obtenu $\geq 26$ points sur 60 points (note totale) au début de la tentative mais au contraire après avoir terminé la tentative, $70 \%$ du groupe de l'étude $(\mathrm{n}=$ 23) ont obtenu $\geq 58$ sur 60 . Cela veut dire que $70 \%$ du groupe de l'étude ont obtenu ( $96.6 \%$ de la note totale). Ce progrès considérable est du à l'intervention par le programme proposé, ce qui a assuré l'efficacité de l'approche de l'enseignement différencié pour remédier aux difficultés linguistiques chez les sujets du groupe de l'étude.

\section{Interprétation du résultat pour les difficultés linguistiques en général.}

Le résultat a montré de façon claire qu'il existe une différence entre les moyennes des notes des apprenants(es) pour les items du test en entier en faveur de la post-application. Ce qui a expliqué le rôle de l'approche de l'enseignement différencié et de ses stratégies employées pendant les séances de la tentative. La stratégie de (PCP) avec les autres adoptées à la lueur de l'approche donnent aux apprenants(es) de FLÉ l'occasion à réfléchir de façon individuelle à chaque difficulté, ensuite à consulter ou bien à comparer sa réponse avec celle de son duo, et à la fin à partager sa réponse avec toute la classe. Chaque étape suivie par l'apprenant(e) a ajouté une correction ou plutôt une amélioration positive pour sa réponse. Toutes ces étapes font une sorte d'amélioration à tous les niveaux; niveau cognitif où les apprenants(es) peuvent corriger leurs savoirs concernant 
telle ou telle difficulté et où ils(elles) peuvent perfectionner leurs savoirfaire concernant la même difficulté.

L'approche de l'enseignement différencié et ses stratégies telles que la stratégie de (penser-paire-partager) ou (penser-comparer-partager), celle des discussions en groupe ...etc. renforcent le sentiment des apprenants(es) participants(es) qu'ils(elles) deviennent autonomes; ils(elles) ont participé aux cours de façon positive lors du processus de l'enseignement / apprentissage et notamment lors du traitement de telle ou telle difficulté linguistique et que c'était à eux(elles)-mêmes de faire face à leurs lacunes, leurs difficultés linguistiques pour maîtriser ces compétences linguistiques comme une étape importante de maîtriser la langue française oralement et par écrit.

La même approche de l'enseignement différencié ont conduit les apprenants(es) de FLÉ de ce niveau d'apprentissage à changer leurs mentalités concernant le processus de la didactique de FLÉ en général et de la didactique des difficultés linguistiques en particulier; après la tentative ces enseignés(es) de FLÉ deviennent convaincus(es) de la nécessité de diagnostiquer leurs lacunes par eux-mêmes et deviennent de plus en plus capables d'y apporter des solutions par la voie des ressources disponibles soit des livres soit des discussions avec leurs collègues en classe de langue.

La même approche et ses stratégies adoptées dans cette étude fait de l'apprenant(e) quelqu'un d'actif tout au long de la tentative; il(elle) a réfléchi, il(elle) a essayé de répondre aux questions et aux activités proposées, il(elle) a cherché des solutions à leurs problèmes et à leurs difficultés dans les ressources essentielles lors de son retour chez lui à la fin de la journée, il(elle) a consulté ses pairs en classe, ainsi il(elle) a 
consulté les livres et les dictionnaires relatifs pour remédier à ses propres lacunes et ses propres difficultés. Notre apprenant(e) devient donc quelqu'un d'autonome.

Pour la vérification de la deuxième hypothèse.

Cette deuxième hypothèse est : «Il existe une différence statistiquement signifiante entre les moyennes des notes des sujets du groupe de l'étude en ce qui concerne les difficultés d'ordre grammatical dans les résultats de la pré-post application du test des difficultés linguistiques au seuil de (0.01) en faveur de la post application». Pour vérifier une telle hypothèse, on a traité les notes brutes de l'application du test des difficultés linguistiques avant et après la tentative par la technique statistique intitulée «SPSS» et on a obtenu les valeurs contenues dans le tableau ci-dessous :-

Tableau no. ( 4 ).

Résultats relatifs aux items relatifs aux difficultés grammaticales.

\begin{tabular}{|c|c|c|c|c|c|c|c|}
\hline $\begin{array}{c}\text { Difficulté } \\
\text { mesurée }\end{array}$ & $\begin{array}{c}\text { Applicatio } \\
\mathrm{n} \text { du test. }\end{array}$ & $\begin{array}{c}\text { Nombr } \\
\text { e du } \\
\text { groupe. }\end{array}$ & $\begin{array}{l}\text { Moyenne } \\
\text { arithmétiqu } \\
\text { e. } \\
\text { (M.) }\end{array}$ & $\begin{array}{l}\text { Écart } \\
\text { type. }\end{array}$ & $\begin{array}{l}\text { Valeur } \\
\text { de T. } \\
\text { calculée }\end{array}$ & $\begin{array}{c}\text { Valeu } \\
\mathrm{r} \text { de/d' } \\
\mathrm{ETA}^{2} \\
(\eta)\end{array}$ & $\begin{array}{l}\text { S. } \\
\text { ou } \\
\text { NS. }\end{array}$ \\
\hline \multirow{2}{*}{$\begin{array}{c}\text { Difficultés } \\
\text { d'ordre } \\
\text { grammatica } \\
1 .\end{array}$} & Pré - test. & \multirow[b]{2}{*}{33} & 5.0303 & $\begin{array}{c}1.1315 \\
0\end{array}$ & \multirow[b]{2}{*}{19.553} & \multirow[b]{2}{*}{0.92} & \multirow{2}{*}{$\begin{array}{c}\text { S. } \\
\text { au } \\
\text { seui } \\
1 \mathrm{de} \\
0.01\end{array}$} \\
\hline & Post - test. & & 10.4848 & $\begin{array}{c}2.3994 \\
9\end{array}$ & & & \\
\hline
\end{tabular}




\section{Commentaire du tableau.}

Le tableau no. ( 4 ) montre le nombre du groupe de l'étude ( 33 ), la moyenne arithmétique pour la pré-application des items des difficultés grammaticales contenues dans le test $=(5.0303)$, celle pour la postapplication des mêmes items des difficultés grammaticales $=(10.4848)$, l'Écart type pour la pré-application des mêmes difficultés = (1.13150), celui pour la post-application des mêmes items $=(2.39949)$, la valeur de «T» calculée $=(19.553)$, la valeur d'ETA ${ }^{2}=(0.92)$, et l'indice de signifiance $($ S. ou NS.) $=$ S. au seuil de $(0.01)$.

Ces valeurs ont assuré qu'il existe une différence signifiante au seuil de (0.01) entre les moyenne des notes des apprenants(es) du groupe de l'étude en ce qui concerne les items des difficultés grammaticales. Ce qui a assuré l'efficacité du programme proposé pour surmonter les difficultés grammaticales envisagées par les sujets du groupe de l'étude. Or, la deuxième hypothèse de l'étude actuelle a été vérifiée. Cela signifie que le traitement des difficultés grammaticales telles que l'emploi des connecteurs (et - ou - mais, tandis que, bien que ....etc.), la conjugaison correcte des verbes au présent, au passé composé, au futur proche, au futur simple de l'indicatif, la conjugaison des verbes aux modes différents notamment les modes de l'indicatif, du subjonctif et du conditionnel ...etc., à travers les apports de l'approche de l'enseignement différencié était efficace. Cela s'est apparu clairement à travers le schéma statistique individuel ci-dessous où on a remarqué une comparaison entre les niveaux des sujets du groupe de l'étude avant et après la tentative en ce qui concerne les difficultés grammaticales. 

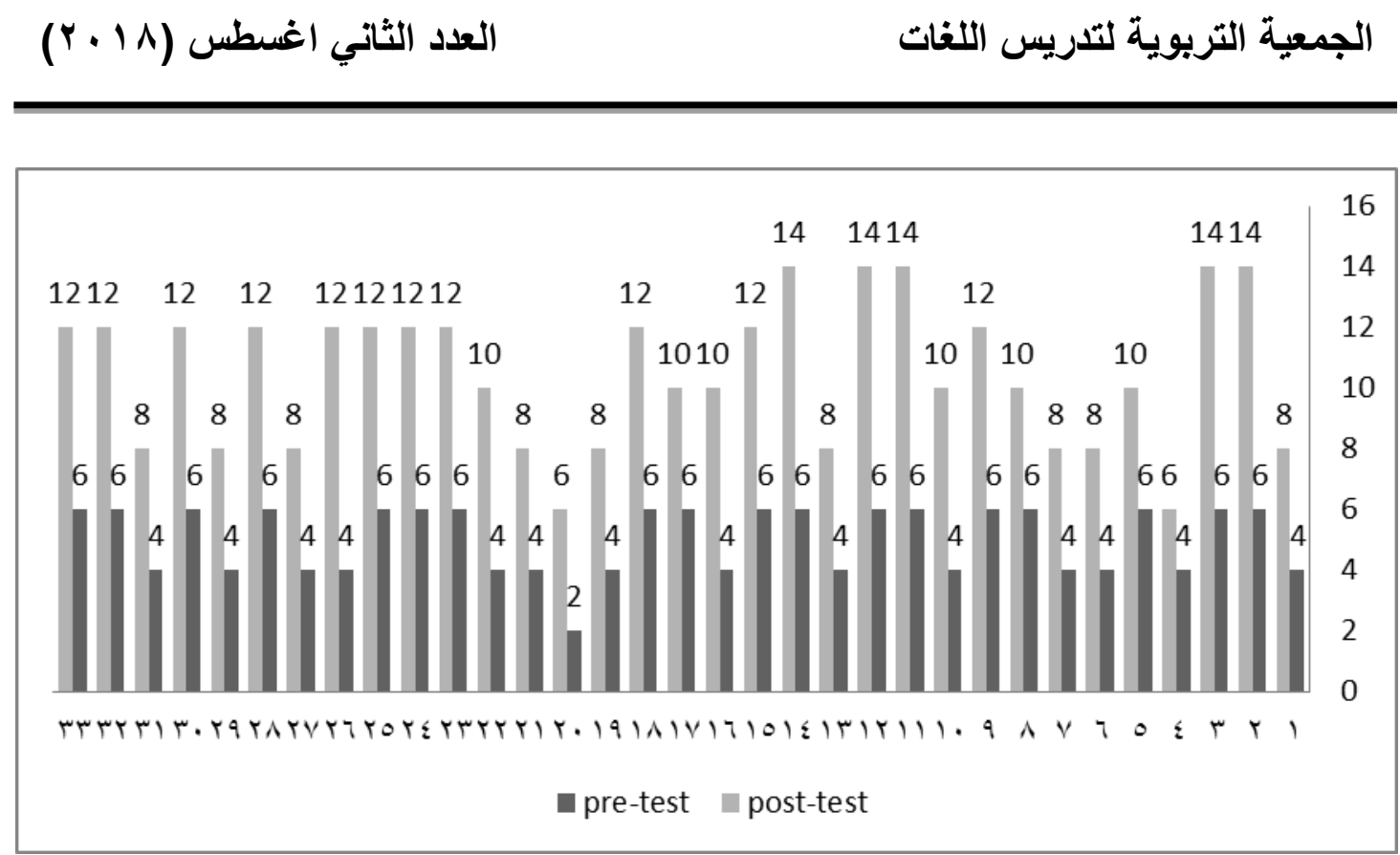

Analyse qualificative du résultat des apprenants(es) pour les difficultés grammaticales.

Le chercheur a observé, à travers le schéma ci-dessus, qu'avant la tentative la majorité massive des points des sujets du groupe est entre (2 et 6 points) dans les items consacrés aux difficultés grammaticales. Après avoir terminé la tentative, ces points sont clairement améliorés; quelques uns(es) des sujets du groupe de l'étude ont obtenu (5 apprenants(es)) ont obtenu 14 points sur 14 comme note totale (11 apprenants(es)) ont obtenu 12 points sur 14, (6 apprenants(es)) ont obtenu 10 points sur 14, (9 apprenants(es)) ont obtenu 8 points sur 14, (2 apprenants(es)) ont obtenu 6 points sur 14. Le chercheur a noté que :-

- $\quad 45 \%$ du groupe de l'étude $(n=15)$ ont obtenu $\geq 4$ points sur 14 points (note totale) consacrés aux items de difficultés grammaticales au début de la tentative mais au contraire après avoir terminé la tentative, $45 \%$ du groupe de l'étude $(\mathrm{n}=15)$ ont obtenu $\geq 10$ points sur 14 . Cela veut dire que $45 \%$ du groupe de l'étude ont obtenu (71.4\% de la note totale). Ce progrès 
considérable est du à l'application du programme élaboré, ce qui a assuré l'efficacité de l'approche de l'enseignement différencié.

- $55 \%$ du groupe de l'étude $(\mathrm{n}=18)$ ont obtenu $\geq 6$ points sur 14 points (note totale) consacrés aux items de difficultés grammaticales au début de la tentative mais au contraire après avoir terminé la tentative, $55 \%$ du groupe de l'étude $(\mathrm{n}=18)$ ont obtenu $\geq 14$ sur 14 . Cela veut dire que $55 \%$ du groupe de l'étude ont obtenu (plus de $96 \%$ de la note totale). Ce progrès considérable est du à l'intervention par le programme proposé, ce qui a assuré l'efficacité de l'approche de l'enseignement différencié pour remédier les difficultés grammaticales chez les sujets du groupe de l'étude.

\section{Pour la vérification de la troisième hypothèse.}

Cette troisième hypothèse est : «Il existe une différence statistiquement signifiante entre les moyennes des notes des sujets du groupe de l'étude en ce qui concerne les difficultés d'ordre structural dans les résultats de la pré-post application du test des difficultés linguistiques au seuil de (0.01) en faveur de la post application». Pour vérifier une telle hypothèse, on a traité les notes brutes de l'application du test des difficultés linguistiques avant et après la tentative par la technique statistique intitulée «SPSS» et on a obtenu les valeurs contenues dans le tableau ci-dessous :- 
Tableau no. ( 5 ).

Résultats relatifs aux items relatifs aux difficultés structurales.

\begin{tabular}{|c|c|c|c|c|c|c|c|}
\hline $\begin{array}{c}\text { Difficulté } \\
\text { mesurée }\end{array}$ & $\begin{array}{c}\text { Applicatio } \\
\text { n du test. }\end{array}$ & $\begin{array}{l}\text { Nombr } \\
\text { e du } \\
\text { groupe. }\end{array}$ & $\begin{array}{c}\text { Moyenne } \\
\text { arithmétique } \\
\text { · } \\
\text { (M.) }\end{array}$ & $\begin{array}{l}\text { Écart } \\
\text { type. }\end{array}$ & $\begin{array}{c}\text { Valeur } \\
\text { de T. } \\
\text { calculée } \\
\text {. }\end{array}$ & $\begin{array}{c}\text { Valeu } \\
\text { r de/d' } \\
\text { ETA }^{2} \\
(\eta)\end{array}$ & $\begin{array}{c}\text { S. } \\
\text { ou } \\
\text { NS. }\end{array}$ \\
\hline \multirow{2}{*}{$\begin{array}{l}\text { Difficultés } \\
\text { structurales }\end{array}$} & Pré - test. & \multirow{2}{*}{33} & 4.5455 & $\begin{array}{c}1.1481 \\
2\end{array}$ & \multirow[b]{2}{*}{23.700} & \multirow[b]{2}{*}{0.95} & \multirow{2}{*}{$\begin{array}{c}\text { S. } \\
\text { au } \\
\text { seui } \\
1 \mathrm{de} \\
0.01\end{array}$} \\
\hline & Post - test. & & 9.6463 & $\begin{array}{c}0.7833 \\
5\end{array}$ & & & \\
\hline
\end{tabular}

\section{Commentaire du tableau.}

Le tableau no. ( 5 ) montre le nombre du groupe de l'étude ( 33 ), la moyenne arithmétique pour la pré-application des items des difficultés structurales contenues dans le test des difficultés linguistiques $=(4.5455)$, celle pour la post-application des mêmes items du test $=(9.6463)$, l'Écart type pour la pré-application des mêmes items $=(1.14812)$, celui pour la post-application des mêmes items $=(0.78335)$, la valeur de $« \mathrm{~T} »$ calculée $=(23.700)$, la valeur d'ETA ${ }^{2}=(0.95)$, et l'indice de signifiance $(\mathrm{S}$. ou NS. $)=$ S. au seuil de $(0.01)$.

Ces valeurs ont assuré qu'il existe une différence signifiante au seuil de (0.01) entre les moyenne des notes des apprenants(es) du groupe de l'étude pour ce qui concerne les difficultés structurales. Ce qui a assuré l'efficacité du programme proposé pour surmonter les difficultés structurales envisagées par les sujets du groupe de l'étude. Or, la troisième hypothèse de l'étude actuelle est vérifiée. Cela signifie que le traitement des difficultés structurales telles l'emploi des connecteurs, 
l'emploi des verbes aux modes et aux temps convenables, l'emploi des morphèmes grammaticaux et des morphèmes lexicaux ...etc. au sein des phrases françaises et les mettre dans des structures logiques et correctes à travers les apports de l'approche de l'enseignement différencié était efficace. Cela s'est apparu clairement à travers le schéma statistique individuel suivant où on a remarqué une comparaison entre les niveaux des sujets du groupe de l'étude avant et après la tentative en ce qui concerne les difficultés structurales.

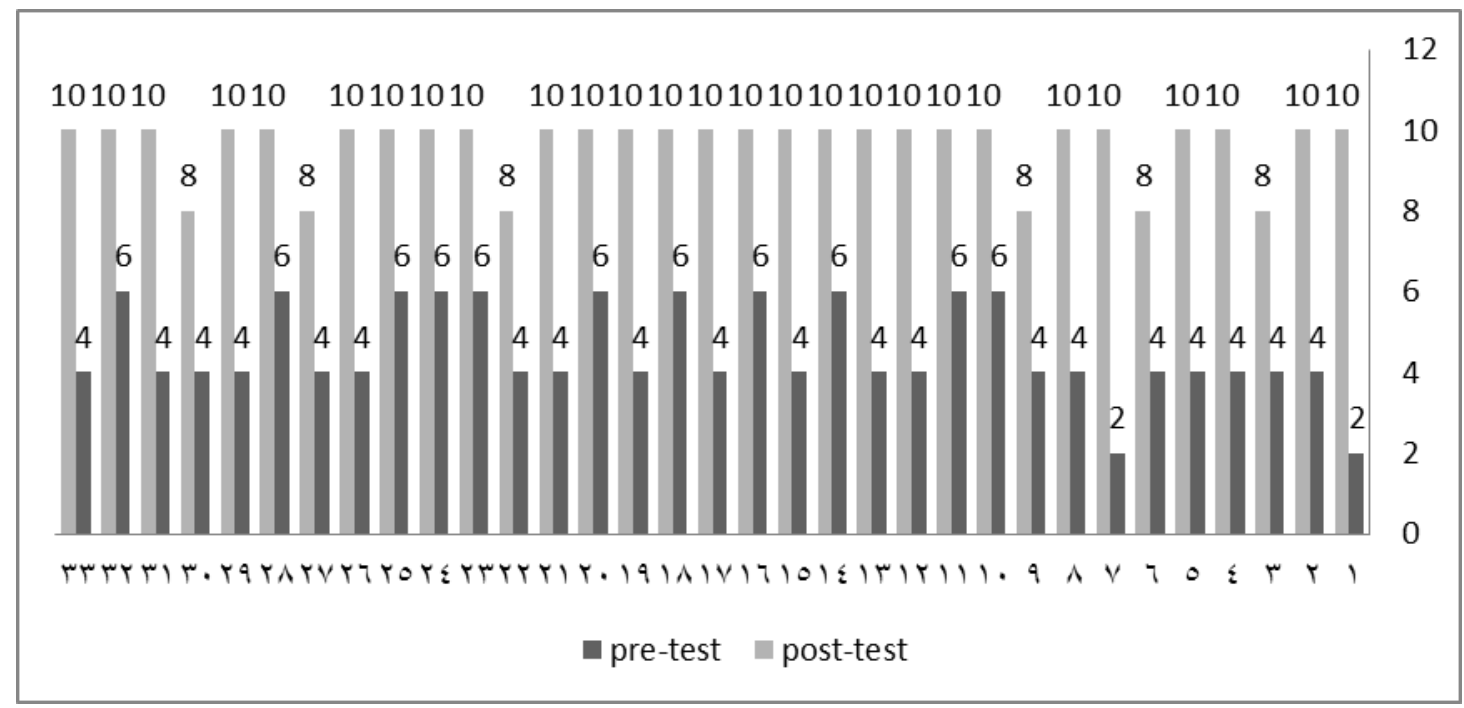

Analyse qualificative du résultat des apprenants(es) pour les difficultés structurales.

On a observé, à travers le schéma ci-dessus, qu'avant la tentative la majorité massive des points des sujets du groupe est entre ( 2 et 6 points) dans les items de difficultés structurales. Après avoir terminé la tentative, ces points sont clairement améliorés; les points sont devenus entre (8 et 10 points) de 10 comme note totale consacrée aux items de ce propos. Cette amélioration est grâce à l'emploi du programme manipulé pendant la période de l'intervention. Le chercheur a noté que :- 
- $67 \%$ du groupe de l'étude $(n=22)$ ont obtenu $\geq 4$ points sur 10 points (note totale) consacrés aux items des difficultés structurales au début de la tentative mais au contraire après avoir terminé la tentative, $24 \%$ du groupe de l'étude $(\mathrm{n}=6)$ ont obtenu 8 points sur 10 . Cela veut dire que $24 \%$ du groupe de l'étude ont obtenu ( $80 \%$ de la note totale). Ce progrès considérable est du à l'application du programme élaboré, ce qui a assuré l'efficacité de l'approche de l'enseignement différencié.

- $33 \%$ du groupe de l'étude $(n=11)$ ont obtenu $\geq 6$ points sur 10 points (note totale) au début de la tentative mais au contraire après avoir terminé la tentative, $76 \%$ du groupe de l'étude $(\mathrm{n}=$ 27) ont obtenu $\geq 10$ sur 10 . Cela veut dire que $76 \%$ du groupe de l'étude ont obtenu ( $100 \%$ de la note totale). Ce progrès considérable est du à l'intervention par le programme proposé, ce qui a assuré l'efficacité de l'approche de l'enseignement différencié pour remédier aux difficultés structurales chez les sujets du groupe de l'étude.

\section{Pour la vérification de la quatrième hypothèse.}

Cette quatrième hypothèse est : «Il existe une différence statistiquement signifiante entre les moyennes des notes des sujets du groupe de l'étude en ce qui concerne les difficultés d'ordre morphologique dans les résultats de la pré-post application du test des difficultés linguistiques au seuil de (0.01) en faveur de la post application». Pour vérifier une telle hypothèse, on a traité les notes brutes de l'application du test des difficultés linguistiques avant et après la tentative par la technique statistique intitulée «SPSS» et on a obtenu les valeurs contenues dans le tableau ci-dessous :- 
Tableau no. ( 6 ).

Résultats relatifs aux items concernant les difficultés morphologiques.

\begin{tabular}{|c|c|c|c|c|c|c|c|}
\hline $\begin{array}{l}\text { Difficulté } \\
\text { mesurée }\end{array}$ & $\begin{array}{l}\text { Applicatio } \\
\text { n du test. }\end{array}$ & $\begin{array}{l}\text { Nombr } \\
\text { e du } \\
\text { groupe. }\end{array}$ & $\begin{array}{l}\text { Moyenne } \\
\text { arithmétique } \\
\text { (M.) }\end{array}$ & $\begin{array}{l}\text { Écart } \\
\text { type. }\end{array}$ & $\begin{array}{l}\text { Valeur } \\
\text { de } \mathrm{T} \text {. } \\
\text { calculée } \\
\text {. }\end{array}$ & $\begin{array}{l}\text { Valeu } \\
\text { r de/d' } \\
\text { ETA }^{2} \\
(\eta)\end{array}$ & $\begin{array}{l}\text { S. } \\
\text { ou } \\
\text { NS. }\end{array}$ \\
\hline \multirow[t]{2}{*}{$\begin{array}{l}\text { Difficultés } \\
\text { morphologiques }\end{array}$} & Pré - test. & \multirow[t]{2}{*}{33} & 5.8788 & $\begin{array}{l}1.1112 \\
4\end{array}$ & \multirow[t]{2}{*}{31.031} & \multirow[t]{2}{*}{0.97} & \multirow{2}{*}{$\begin{array}{l}\text { S. } \\
\text { au } \\
\text { seui } \\
1 \mathrm{de} \\
0.01\end{array}$} \\
\hline & Post - test. & & 13.5758 & $\begin{array}{l}1.6399 \\
4\end{array}$ & & & \\
\hline
\end{tabular}

\section{Commentaire du tableau.}

Le tableau no. ( 6 ) montre le nombre du groupe de l'étude ( 33 ), la moyenne arithmétique pour la pré-application des items de difficultés morphologiques contenues dans le test des difficultés linguistiques $=$ (5.8788), celle pour la post-application des items de difficultés morphologiques du test des difficultés linguistiques $=(13.5758)$, l'Écart type pour la pré-application des mêmes items du test $=(1.11124)$, celui pour la post-application des mêmes items du test $=(1.63994)$, la valeur de

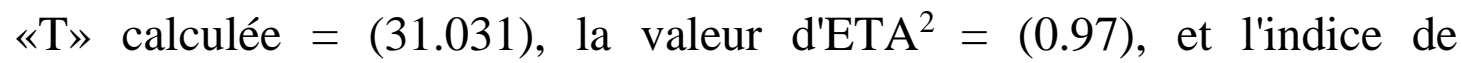
signifiance (S. ou NS.) $=$ S. au seuil de (0.01) .

Ces valeurs ont assuré qu'il existe une différence signifiante au seuil de (0.01) entre les moyenne des notes des apprenants(es) du groupe de l'étude. Ce qui a assuré l'efficacité du programme proposé pour surmonter les difficultés morphologiques envisagées par les sujets du groupe de 
l'étude. Or, la quatrième hypothèse de l'étude actuelle était vérifiée. Cela signifie que le traitement des difficultés morphologiques telles que l'emploi des morphèmes grammaticaux comme l'emploi des articles définis, indéfinis, partitifs ...etc. au même titre que l'emploi des morphèmes lexicaux concernant le lexique des thématiques de la vie courante (la vie scolaire, la vie à la campagne et les vêtement et la mode à travers les apports de l'approche de l'enseignement différencié était efficace. Cela s'est apparu clairement à travers le schéma statistique individuel suivant où on a remarqué une comparaison entre les niveaux des sujets du groupe de l'étude avant et après la tentative en ce qui concerne les difficultés morphologiques.

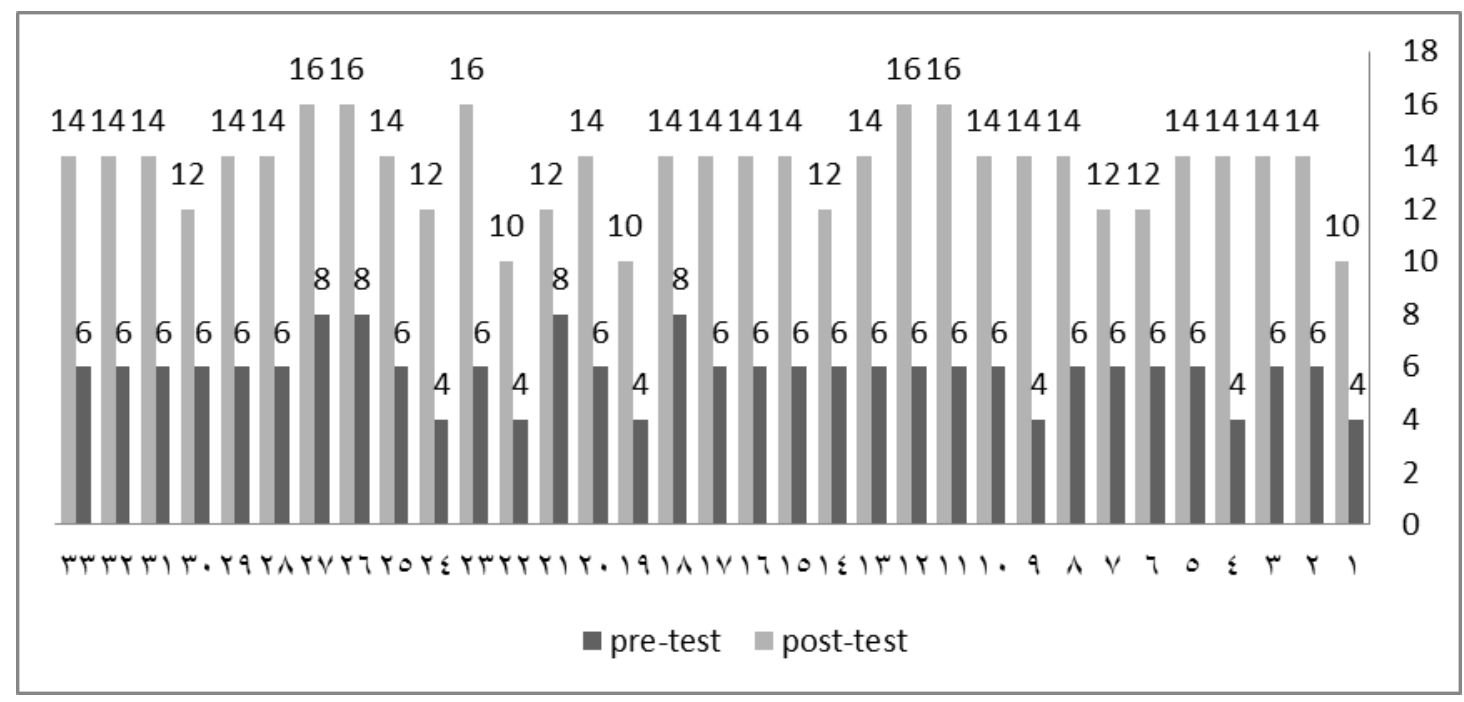

Analyse qualificative du résultat des apprenants(es) pour les difficultés morphologiques.

On a observé, à travers le schéma ci-dessus, qu'avant la tentative la majorité massive des points des sujets du groupe est entre (4 et 8 points) dans les items de difficultés morphologiques. Après avoir terminé la tentative, ces points sont clairement améliorés; les points sont devenus 
entre (10 et 16 points) de 16 comme note totale consacrée aux items de ce propos. Le chercheur a noté que :-

- $18 \%$ du groupe de l'étude $(n=6)$ ont obtenu $\geq 4$ points sur 16 points (note totale) dans les items consacrés aux difficultés morphologiques au début de la tentative mais au contraire après avoir terminé la tentative, $27 \%$ du groupe de l'étude $(\mathrm{n}=9)$ ont obtenu $\geq 10$ points sur 16 . Cela veut dire que $10 \%$ du groupe de l'étude ont obtenu (62.5\% de la note totale). Ce progrès considérable est du à l'application du programme élaboré, ce qui a assuré l'efficacité de l'approche de l'enseignement différencié.

- $82 \%$ du groupe de l'étude $(\mathrm{n}=27)$ ont obtenu $\geq 8$ points sur 16 points (note totale) au début de la tentative mais au contraire après avoir terminé la tentative, $73 \%$ du groupe de l'étude $(\mathrm{n}=$ 24) ont obtenu $\geq 16$ sur 16 . Cela veut dire que $73 \%$ du groupe de l'étude ont obtenu (plus de 95\% de la note totale). Ce progrès considérable est du à l'intervention par le programme proposé, ce qui a assuré l'efficacité de l'approche de l'enseignement différencié pour remédier aux difficultés morphologiques chez les sujets du groupe de l'étude.

\section{Pour la vérification de la cinquième hypothèse.}

Cette cinquième hypothèse est : «Il existe une différence statistiquement signifiante entre les moyennes des notes des sujets du groupe de l'étude en ce qui concerne les difficultés d'ordre lexical dans les résultats de la pré-post application du test des difficultés linguistiques au seuil de (0.01) en faveur de la post application». Pour vérifier une telle 
hypothèse, on a traité les notes brutes de l'application du test de difficultés linguistiques avant et après la tentative par la technique statistique intitulée «SPSS» et on a obtenu les valeurs contenues dans le tableau ci-dessous :-

Tableau no. ( 7 ).

Résultats relatifs aux items concernant les difficultés lexicales.

\begin{tabular}{|c|c|c|c|c|c|c|c|}
\hline $\begin{array}{l}\text { Difficulté } \\
\text { mesurée }\end{array}$ & $\begin{array}{l}\text { Applicatio } \\
\text { n du test. }\end{array}$ & $\begin{array}{l}\text { Nombr } \\
\text { e du } \\
\text { groupe. }\end{array}$ & $\begin{array}{l}\text { Moyenne } \\
\text { arithmétique } \\
\text { (M.) }\end{array}$ & $\begin{array}{l}\text { Écart } \\
\text { type. }\end{array}$ & $\begin{array}{l}\text { Valeur } \\
\text { de } \mathrm{T} . \\
\text { calculée } \\
\text {. }\end{array}$ & $\begin{array}{l}\text { Valeu } \\
\text { r de/d' } \\
\text { ETA }^{2} \\
(\eta)\end{array}$ & $\begin{array}{l}\text { S. } \\
\text { ou } \\
\text { NS. }\end{array}$ \\
\hline \multirow[t]{2}{*}{$\begin{array}{l}\text { Difficulté } \\
\text { s } \\
\text { lexicales. }\end{array}$} & Pré - test. & \multirow[t]{2}{*}{33} & 3.3939 & $\begin{array}{l}1.5398 \\
7\end{array}$ & \multirow{2}{*}{22.523} & \multirow{2}{*}{0.94} & \multirow{2}{*}{$\begin{array}{l}\text { S. } \\
\text { au } \\
\text { seui } \\
1 \text { de } \\
0.01\end{array}$} \\
\hline & Post - test. & & 8.0606 & $\begin{array}{l}1.6944 \\
7\end{array}$ & & & \\
\hline
\end{tabular}

\section{Commentaire du tableau.}

Le tableau no. ( 7 ) montre le nombre du groupe de l'étude ( 33 ), la moyenne arithmétique pour la pré-application du test des difficultés linguistiques $=(3.3939)$, celle pour la post-application des items des difficultés lexicales contenues dans le test de difficultés linguistiques $=$ (8.0606), l'Écart type pour la pré-application des mêmes items du test $=$ (1.53987), celui pour la post-application des mêmes items du test $=$ (1.69447), la valeur de «T» calculée $=(22.523)$, la valeur d'ETA ${ }^{2}=$ (0.94), et l'indice de signifiance $($ S. ou NS.) $=$ S. au seuil de $(0.01)$.

Ces valeurs ont assuré qu'il existe une différence signifiante au seuil de (0.01) entre les moyenne des notes des apprenants(es) du groupe de l'étude. Ce qui a assuré l'efficacité du programme proposé pour remédier 
aux difficultés lexicales envisagées par les sujets du groupe de l'étude. Or, la cinquième hypothèse de l'étude actuelle était vérifiée. Cela signifie que le traitement des difficultés lexicales notamment celles concernant le lexique relatif aux thèmes de la vie scolaire, la vie à la campagne, et les vêtements et la mode à travers les apports de l'approche de l'enseignement différencié était efficace. Cela s'est apparu clairement à travers le schéma statistique individuel suivant où on a remarqué une comparaison entre les niveaux des sujets du groupe de l'étude avant et après la tentative en ce qui concerne les difficultés lexiques.

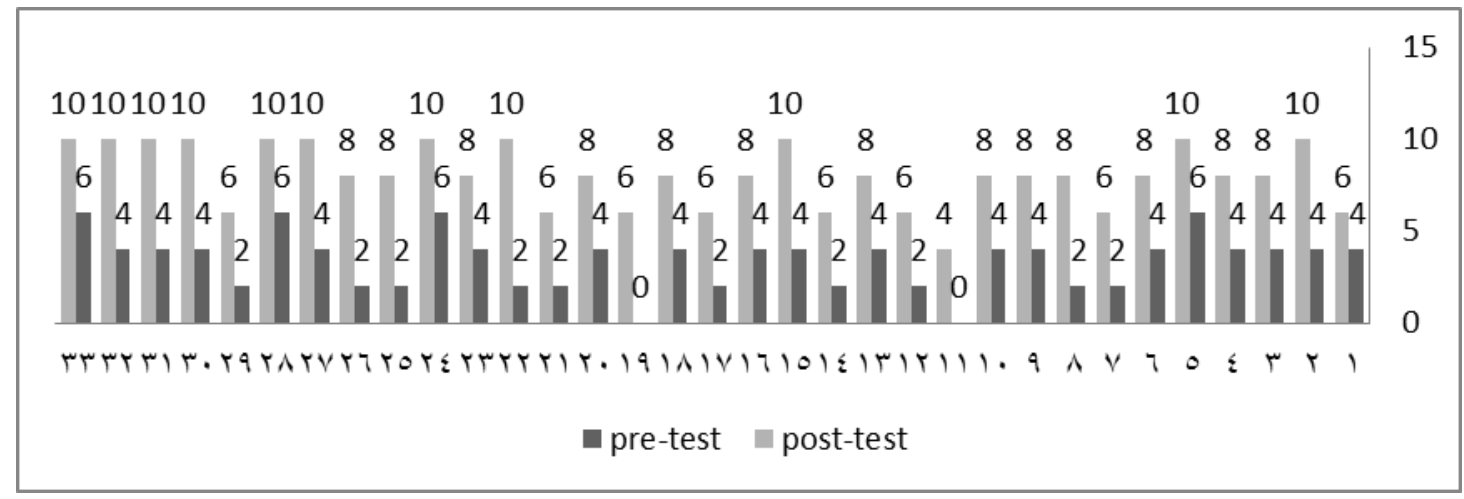

Analyse qualificative du résultat des apprenants(es) pour les difficultés lexicales.

On a observé, à travers le schéma ci-dessus, qu'avant la tentative la majorité massive des points des sujets du groupe est entre ( 0 et 6 points) dans les items de difficultés lexicales. Après avoir terminé la tentative, ces points sont clairement améliorés; les points sont devenus entre (6 et 10 points) de 10 comme note totale consacrée aux items de ce propos. Le chercheur a noté que :-

- $84 \%$ du groupe de l'étude $(n=28)$ ont obtenu $\geq 4$ points sur 10 points (note totale) dans les items consacrés aux difficultés lexicales au début de la tentative mais au contraire après avoir 
terminé la tentative, $58 \%$ du groupe de l'étude $(\mathrm{n}=19)$ ont obtenu $\geq 8$ points sur 10 . Cela veut dire que $58 \%$ du groupe de l'étude ont obtenu ( $80 \%$ de la note totale). Ce progrès considérable est du à l'application du programme élaboré, ce qui a assuré l'efficacité de l'approche de l'enseignement différencié.

- $15 \%$ du groupe de l'étude $(n=5)$ ont obtenu $\geq 6$ points sur 10 points (note totale) au début de la tentative mais au contraire après avoir terminé la tentative, $43 \%$ du groupe de l'étude $(\mathrm{n}=$ 14) ont obtenu $\geq 10$ sur 10 . Cela veut dire que $43 \%$ du groupe de l'étude ont obtenu ( $100 \%$ de la note totale). Ce progrès considérable est du à l'intervention par le programme proposé, ce qui a assuré l'efficacité de l'approche de l'enseignement différencié pour remédier aux difficultés lexicales chez les sujets du groupe de l'étude.

\section{Pour la vérification de la sixième hypothèse.}

Cette sixième hypothèse est : «Il existe une différence statistiquement signifiante entre les moyennes des notes des sujets du groupe de l'étude en ce qui concerne les difficultés d'ordre sémantique dans les résultats de la pré-post application du test de difficultés linguistiques au seuil de (0.01) en faveur de la post application». Pour vérifier une telle hypothèse, on a traité les notes brutes de l'application du test des difficultés linguistiques avant et après la tentative par la technique statistique intitulée «SPSS» et on a obtenu les valeurs contenues dans le tableau ci-dessous :- 
Tableau no. ( 8 ).

Résultats relatifs aux items concernant les difficultés sémantiques.

\begin{tabular}{|c|c|c|c|c|c|c|c|}
\hline $\begin{array}{l}\text { Difficulté } \\
\text { mesurée }\end{array}$ & $\begin{array}{l}\text { Applicatio } \\
\text { n du test. }\end{array}$ & $\begin{array}{l}\text { Nombr } \\
\text { e du } \\
\text { groupe. }\end{array}$ & $\begin{array}{l}\text { Moyenne } \\
\text { arithmétiqu } \\
\text { e. } \\
\text { (M.) }\end{array}$ & $\begin{array}{l}\text { Écart } \\
\text { type. }\end{array}$ & \begin{tabular}{l}
\multicolumn{2}{l}{ Valeur } \\
de $\mathrm{T}$. \\
calculée \\
.
\end{tabular} & $\begin{array}{l}\text { Valeu } \\
\text { r de/d' } \\
\text { ETA }^{2} \\
(\eta)\end{array}$ & $\begin{array}{l}\text { S. } \\
\text { ou } \\
\text { NS. }\end{array}$ \\
\hline \multirow[t]{2}{*}{$\begin{array}{l}\text { Difficultés } \\
\text { sémantique } \\
\text { s. }\end{array}$} & Pré - test. & \multirow[t]{2}{*}{33} & 2.9091 & $\begin{array}{l}1.2339 \\
9\end{array}$ & \multirow[t]{2}{*}{13.203} & \multirow[t]{2}{*}{0.84} & \multirow{2}{*}{$\begin{array}{l}\text { S. } \\
\text { au } \\
\text { seui } \\
1 \text { de } \\
0.01\end{array}$} \\
\hline & Post - test. & & 6.9697 & $\begin{array}{l}1.8789 \\
1\end{array}$ & & & \\
\hline
\end{tabular}

\section{Commentaire du tableau.}

Le tableau no. ( 8 ) montre le nombre du groupe de l'étude ( 33 ), la moyenne arithmétique pour la pré-application des items des difficultés sémantiques contenues dans le test des difficultés linguistiques = (2.9091), celle pour la post-application des mêmes items du test $=$ (6.9697), l'Écart type pour la pré-application du test $=(1.23399)$, celui pour la post-application des mêmes items du test $=(1.87891)$, la valeur de «T» calculée $=(13.203)$, la valeur $\mathrm{d}^{\prime} \mathrm{ETA}^{2}=(0.84)$, et l'indice de signifiance $($ S. ou NS.) $=$ S. au seuil de $(0.01)$.

Ces valeurs assure qu'il existe une différence signifiante au seuil de (0.01) entre les moyenne des notes des apprenants(es) du groupe de l'étude. Ce qui a assuré l'efficacité du programme proposé pour surmonter les difficultés sémantiques envisagées par les sujets du groupe de l'étude notamment celles relatives aux synonymes et aux antonymes des termes 
concernant la vie scolaire, la vie à la campagne, et le thème des vêtements et la mode. Or, la sixième hypothèse de l'étude actuelle était vérifiée. Cela signifie que le traitement des difficultés sémantiques à travers les apports de l'approche de l'enseignement différencié était efficace. Cela s'est apparu clairement à travers le schéma statistique individuel suivant où on a remarqué une comparaison entre les niveaux des sujets du groupe de l'étude avant et après la tentative en ce qui concerne les difficultés sémantiques.

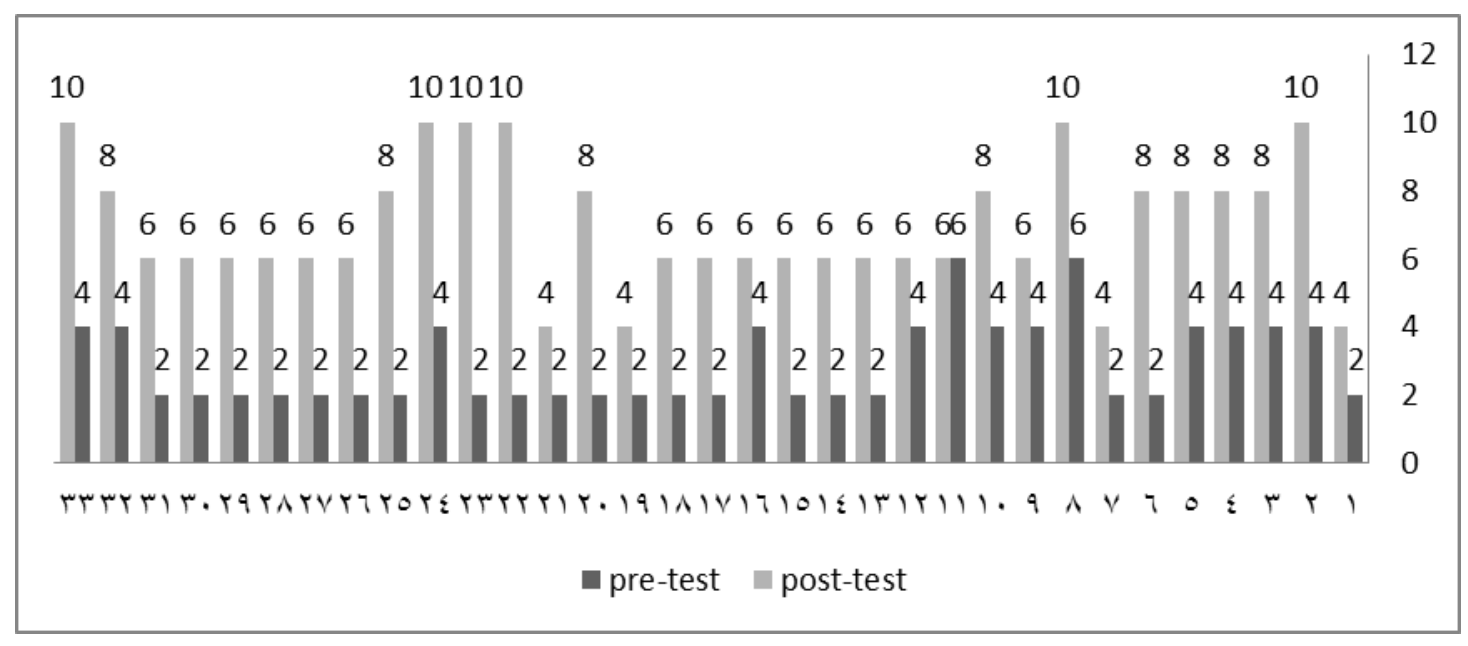

Analyse qualificative du résultat des apprenants(es) pour les difficultés sémantiques.

On a observé, à travers le schéma ci-dessus, qu'avant la tentative la majorité massive des points des sujets du groupe est entre ( 2 et 6 points) dans les items de difficultés sémantiques. Après avoir terminé la tentative, ces points sont clairement améliorés; les points sont devenus entre (6 et 10 points) de 10 comme note totale consacrée aux items de ce propos. Le chercheur a noté que :-

- $94 \%$ du groupe de l'étude $(\mathrm{n}=31)$ ont obtenu $\geq 4$ points sur 10 points (note totale) dans les items consacrés aux difficultés 
sémantiques au début de la tentative mais au contraire après avoir terminé la tentative, $58 \%$ du groupe de l'étude $(\mathrm{n}=19)$ ont obtenu $\geq 6$ points sur 10 . Cela veut dire que $10 \%$ du groupe de l'étude ont obtenu (60\% de la note totale). Ce progrès remarquable est du à l'application du programme élaboré, ce qui a assuré l'efficacité de l'approche de l'enseignement différencié.

- $6 \%$ du groupe de l'étude $(\mathrm{n}=2)$ ont obtenu $\geq 6$ points sur 10 points (note totale) dans les items consacrés aux difficultés sémantiques au début de la tentative mais au contraire après avoir terminé la tentative, $32 \%$ du groupe de l'étude $(\mathrm{n}=13)$ ont obtenu $\geq 10$ sur 10 . Cela veut dire que $32 \%$ du groupe de l'étude ont obtenu ( $100 \%$ de la note totale). Ce progrès considérable est du à l'intervention par le programme proposé, ce qui a assuré l'efficacité de l'approche de l'enseignement différencié pour remédier aux difficultés sémantiques chez les sujets du groupe de l'étude.

\section{Commentaire général et interprétation des résultats.}

Après avoir présenté et analysé les résultats de l'étude actuelle par les techniques statistiques propres, on s'est assuré qu'il existe une différence statistiquement signifiante entre les moyennes des notes des sujets du groupe de l'étude dans les résultats de la pré-post application du test de difficultés linguistiques (difficultés relatives aux compétences grammaticales, structurales, morphologiques, lexicales et sémantiques) en faveur de la post-application. Ce résultat général a montré de façon claire que :- 
- Le niveau des apprenants(es) ayant des difficultés linguistiques s'est amélioré grâce à l'emploi du programme élaboré à la lueur de l'approche de l'enseignement différencié du travail telles que la stratégie de (penser-pair-partager) et celle de la discussion en groupe. Des stratégies font des apprenants(es) participants(es) des sujets autonomes et actifs(ves) lors de l'apprentissage

- Ces genres d'apprenants(es) deviennent capables de faire face à leurs obstacles et difficultés linguistiques.

- Ces apprenants(es), à travers l'approche manipulée deviennent de plus en plus indépendants(es); ils (elles) ont eu la capacité à diagnostiquer leurs lacunes et leurs difficultés linguistiques par eux (elles)-mêmes telles que l'emploi des connecteurs soit au sein des phrases simples (et - ou - mais ...etc.) soit au sein des phrases composées (quand - pour que -bien que - afin que ...etc.), la conjugaison correcte des verbes soit aux modes, soit aux temps convenables, l'emploi des adverbes composés (làdessus - là-dessous ...etc.) et des prépositions et les nuances entre elles (sur $v s$ dans - sous $v s$ sur - de $v s$ à ...etc.), l'emploi correct des morphèmes soit lexicaux soit grammaticaux (articles définis et indéfinis ...etc.), l'emploi du lexique convenable aux situations discursives ou d'échanges et l'emploi correct des synonymes, des antonymes au sein des phrases en français.

- L'approche et ses stratégies ont guidé les apprenants(es) à maîtriser le trajet du processus de l'apprentissage de la langue française et se documenter en référant aux ressources pour s'assurer de leurs savoirs linguistiques au même titre de 
perfectionner leurs savoir-faire langagiers; ils(elles) s'apprennent au fur et à mesure à employer les ressources différentes comme les livres de grammaire, de morphologie, les dictionnaires de synonymes ...etc., pour se documenter.

- L'approche en question a donné l'occasion aux apprenants(es) à remédier à leurs difficultés et leurs lapsus linguistiques; ils(elles) consultent les experts soit des partenaires soit des enseignants(es) en cas de nécessité pour s'assurer de leurs savoirs et pour corriger leurs difficultés relatives à tel ou tel savoir-faire linguistique et langagier.

- Les apprenants(es) ont appris à travers une telle approche le sens de responsabilité et le sens de coopération avec leurs pairs pour faire face aux difficultés linguistiques et langagières actuellement et dans le futur.

- Ils(elles) deviennent, après avoir terminé la tentative, capables de diagnostiquer leurs différentes difficultés linguistiques et langagières avant de chercher des solutions à de telles difficultés.

- Ils(elles) deviennent en employant les stratégies de l'approche de l'enseignement différencié notamment celle de (PCP), aptes à réfléchir aux différentes situations problématiques, à consulter leurs pairs ou duos pour chercher des solutions chez eux(elles) et enfin à partager leurs solutions avec toute la classe pour les améliorer.

- On peut donc clôturer que l'emploi de l'approche de l'enseignement différencié et notamment les stratégies de (PCP) 
et de discussion en groupe étaient efficace pour le traitement des difficultés linguistiques notamment les difficultés grammaticales, structurales, morphologiques, lexicales et sémantiques chez les sujets du groupe de l'étude.

\section{Conclusion.}

L'étude actuelle visait dès le début à aider les apprenant(es)s de FLÉ au cycle préparatoire à remédier à leurs difficultés linguistiques couramment commises lors $\mathrm{d}$ se mettre en situations d'échanges en français comme langue étrangère dans les différentes situations de la vie courante. Afin d'atteindre ces objets, on a élaboré des outils de mesure et des autres outils et matériaux d'enseignement/ apprentissage tels qu'un test de diagnostic, une typologie de difficultés linguistiques, un programme d'enseignement, et un guide pédagogique. Le chercheur a standardisé ces outils en employant les techniques et les équations statistiques pour les manipuler au cours de la tentative. Après avoir terminé la tentative, on s'est assuré que le programme proposé en basant sur les apports sous-jacents de l'approche de l'enseignement différencié était efficace pour remédier aux difficultés communes chez les apprenant(es)s de FLÉ au cycle préparatoire.

Cette amélioration du niveau des apprenant(es)s de FLÉ en difficulté linguistique est due à :-

La convenance des unités du programme proposé au niveau langagier des apprenant(es)s du groupe de l'étude.

La convenance des stratégies employées à la lueur de l'approche de l'enseignement différencié aux difficultés traitées. 
L'efficacité des étapes de la stratégie de (PPP) / (PCP) pour traiter les difficultés linguistiques contenues dans le programme.

La typicalité des difficultés contenues dans le programme à celles étant en face de la majorité des sujets de ce niveau d'apprentissage.

La simplicité de la manière du traitement mené dans le programme élaboré.

L'efficacité des discussions opérées dans les petits groupes composés pour apporter remèdes aux difficultés linguistiques traitées à travers le programme.

La suffisance d'activités consacrées à chaque type de difficultés linguistiques traitées dans le programme proposé.

La suffisance du temps consacré à chaque unité au même titre à chaque item traité par le programme proposé.

La simplicité des savoirs linguistiques contenus et présentés à travers le programme proposé.

Le chercheur a touché un changement positif dans le comportement langagier de tous et toutes les apprenant(es)s du groupe de l'étude après avoir terminé la tentative; ils(elles) deviennent plus actifs(actives) qu'auparavant. Ils(elles) peuvent diagnostiquer leurs lacunes linguistiques, déterminer leurs types ensuite peuvent les traiter de façon autonome.

* En un mot, le programme proposé état efficace dans l'amélioration des niveaux des apprenant(es)s de FLÉ de ce niveau d'apprentissage dans les compétences linguistiques constituant des difficultés pour eux(elles). 


\section{Recommandations.}

À la lueur des résultats obtenus à travers l'étude actuelle, le chercheur peut recommander de / d' :-

- Mettre l'accent sur les difficultés linguistiques des apprenants(es) dans tous les niveaux d'apprentissage. Ces difficultés linguistiques constituent des obstacles en face de nos apprenants(es) et on doit essayer au fur et à mesure de les aider à y remédier régulièrement.

- Pousser nos apprenants(es) à diagnostiquer puis à remédier à leurs difficultés linguistiques au même titre qu'à leurs difficultés d'apprentissage de la langue française en général soit au niveau de l'oral soit au niveau de l'écrit.

- Encourager nos apprenants(es) à pratiquer l'apprentissage actif que ce soit pour les compétences linguistiques et leurs difficultés relatives ou pour les compétences paralinguistiques.

- Inciter nos apprenants(es) à développer voire à maîtriser leurs compétences linguistiques et paralinguistiques notamment les compétences coopératives (ils /elles doivent s'enseigner comment comment collaborer avec les autres collègues lors du travail en classe de langue).

- Pousser les enseignants(es) à employer largement l'approche de l'enseignement différencié dans leurs travaux en classe de langue française pour le développement de toutes les compétences langagières chez leurs enseignés(es).

- Pousser les apprenants(es) à employer largement la stratégie de (PPP) ou de (PCP) dans toutes les activités d'apprentissage pour 
développer toutes les compétences langagières (compréhension orale et écrite ou production orale et écrite) chez leurs enseignés(es).

\section{Propositions.}

À la sueur de l'achèvement de l'étude actuelle, le chercheur peut proposer des études futures telles que :-

- Emploi de l'approche de l'enseignement différencié pour développer les compétences de la compréhension créative chez les apprenants(es) de FLÉ au cycle préparatoire.

- Efficacité de la stratégie de Penser-Comparer-Partager pour le développement des compétences de la compréhension et de la production critique chez les apprenants(es) de ce niveau d'apprentissage.

- Programme proposé basé sur l'approche de l'enseignement différencié pour enrichir les compétences sémantiques chez les adolescents(es) débutants(es) en FLÉ.

- Programme d'entrainement proposé à la lueur des apports sousjacents de l'approche de l'enseignement différencié pour le développement des compétences professionnelles chez les enseignants(es) de FLÉ.

- Emploi de la stratégie de (PPP) ou de (PCP) pour améliorer le niveau des apprenants(es) de FLÉ dans les compétences de la compréhension des textes littéraires. 


\section{Bibliographie.}

A) Références en langue française.

Alberta Éducation, (2003). Stratégies d'enseignement : Guide de mise en œuvre, Carrière et vie, Alberta, Canada.

Alberti M., et Lavoine E., (2012). Les Amiffixes : création d'un matériel orthophonique visant l'enrichissement lexical grâce à la morphologie dérivationnelle pour les retards de langage ou leurs séquelles à l'école élémentaire, Mémoire, Université de Lille II.

Baylon C., et Fabré P., (2003). Initiation à la linguistique, Nathan / VUEF, Paris.

Beacco J.-C., (1995). La méthode circulante et les méthodologies constituées, le français dans le monde, Numéro spécial, méthodes et méthodologies, Janvier, PP. 36-41.

Benetou E., (2007). Pour un pédagogie de l'action à travers le conte, (Éd. Proscolli A. \& Forakis K.), actes de la journée d'étude intitulée «Enseigner le français langue étrangère à l'école primaire: méthodes et pratique, Faculté de lettres, Université nationale d'Athènes, PP. 181 194.

Besse H., et Galisson R., (1980). Polémique en didactique : $d u$ renouveau en question, CLÉ international, Paris.

Bérard E., (1991). L'approche communicative : théories et pratiques, CLÉ International, Paris.

Berthes H., (2011). Rôle de la morphologie chez les enfants dyslexiques : étude des effets d'un entraînement morphologique dans le cadre d'une rééducation orthophonique. Mémoire de master en linguistique, Université de Paris-Ouest.

Beuchat L., (2015). La pédagogie différenciée : le point de vue d'enseignants partagés entre conviction et interrogation, mémoire, Haute École Pédagogique (HÉP), BEJUNE.

Brossard L., (1999). Mettre à jour ses savoirs sur l'apprentissage, pour des pratiques pédagogiques revitalisées, bibliothèque nationale $\mathrm{du}$ Québec, Canada.

Boulet M., et Pharand J., (2008). L'accompagnement concerté des stagiaires en enseignement, Presse de l'université du Québec, Canada.

Chanay G., S. D.). Comment mettre en place une pédagogie différenciée au sein d'une classe afin d'assurer les apprentissages auprès de chaque enfant ?, IUFM, Académie de Bourgogne.

Chiss J.-L., (2002). Débats dans l'enseignement / apprentissage de la grammaire, Cahiers de l'ILSL, No. 13, Lausanne, PP. 5 -16. 
Chiss J-L. \& Meleuc S., (2001). Et la grammaire de phrase ?, Le français aujourd'hui, No. 135, AFEF, Paris.

Clavères M.-H., (1985). 1889 ou la lassitude du corps, dans : A. P. L. V. (éd.), le citoyen de langue et les langues, La dimension politique de l'apprentissage des langues, Actes du colloque de Cerisy, Paris.

Colin F., (1880). L'art d'enseigner et d'étudier les langues, Paris.

Collaud A., (2009). Le rôle des informations morphologiques dans l'apprentissage de la lecture en français, Mémoire de maîtrise, Université de Genève.

Conseil de l'Europe (2001). Cadre Européen commun de référence pour les langues : Apprendre, Enseigner, Évaluer, Didier, Disponible sur www.coe.int/lang-CECR.

Conseil Central de l'Enseignement Catholique Primaire (CCECP), (1988). Pour une pédagogie de l'école chrétienne fondamentale aujourd'hui.France.

Culioli A., (1990). Pour une linguistique de l'énonciation : opérations et représentations, Tome I : l'homme dans la langue, Ophrys, Paris.

Cuq J.-P., (2003). Dictionnaire de didactique du français langue étrangère et seconde, Clé international, Paris.

Dalmasso N., (2009). Étude du lien entre la compétence pragmatique et la compétence grammaticale auprès d'apprenants allophones de français L2, mémoire de magistère, Université du Québec, Montréal.

Danescu E., (2012). La différenciation pédagogique comme solution à la réussite scolaire de tous et de toutes, Université de Pitesti.

De Saussure F., (1972). Cours de Linguistique générale, Édition rédigée par Charles Bally et Albert Sechehaye, critiquée par Tullio de Mauro, Payot, Paris.

Descampe S., et al. (2014). Pratiques de pédagogie différenciée à l'école primaire, Administration générale de l'enseignement et de la recherche scientifique, Bruxelles.

Doquin A., (2008). L'enseignement du français aux hispanophones : problèmes repérés, études linguistiques, propositions didactiques, Thèse de PHD., Université de Paris IV.

Duchiron E., (2003). Les technologies de l'information et de la communication dans l'enseignement / apprentissage des langues : Atouts, limites, et exploitations potentielles du choix fourni, mémoire, Faculté de didactique de français langue étrangère, Université de Paris III.

Dufour J.-M., (2005). Apprentissage d'une langue par la dramatisation, Académie de Nancy-Metz, Janvier, Disponible sur www.ac-nancymetz.fr/dramatisation.pdf. 
Dufossé Sournin S., (2007). Les théories linguistiques, les pratiques pédagogiques et l'acquisition de la détermination nominale en anglais chez les apprenants francophones, thèse de PHD., Université de la Réunion, Disponible sur www.tel.archives-ouvertes.fr/tel-00462508.

Dumarest D., et Morsel M.-H., (2005). Le chemin des mots ; pour un apprentissage méthodique du vocabulaire français, presses universitaires de Grenoble, Grenoble. Disponible sur www.pug.fr.

Galisson R., et Coste D., (1979). Dictionnaire de didactique des langues, Hachette, Paris.

Germain C., (1993). Évolution de l'enseignement des langues : 5000 ans d'histoire, CLÉ International, Paris.

Germain C., et Séguin H., (1998). Le point sur la grammaire, Clé international, Paris.

Grégoire M., et Kostucki A., (2012). Grammaire progressive du français, CLÉ international disponible sur www.frenchpdf.com.

Grossmann F., (2012). Le rôle de la compétence lexicale dans le processus de lecture et l'interprétation des textes, Plate-forme Internet sur la littératie, Université de Stendhal, Grenoble III. Disponible sur www.forumlecture.ch.

Grolleau A.,-C., (2010). Comment faire réagir mes étudiants en cours ?, Formations CGE-PDL, Décembre.

Hamon A., (2007). Grammaire et analyse : analyse grammaticale et analyse logique, Hachette Livre, Paris.

Hodel H., (2007). Former les enseignants à l'utilisation du portfolio européen des langues, le cadre européen commun de référence pour les langues : activités, compétences, niveaux, $2^{\text {ème }}$ programme d'activités à moyen terme du CELV (2004-2007).

Houari Meyahi, (2013). Les difficultés de passage de la terminologie linguistique du français vers l'arabe, Synergie Algérie, No. 20, PP. 93 107.

Huot H., (2005). La morphologie, forme et sens des mots du français, Armand Colin, Paris.

Feyfant A., et Gaussel M., (2007). Méthodes de lecture et difficultés d'apprentissage, dossier d'actualité de VST, No. 31, Novembre. Disponible sur www.inrp.fr/vst.

Forget A., (2017). Quels sont les différents types de différenciation pédagogique dans la classe ?, actes de la conférence «différenciation pédagogique», centre national d'évaluation du système scolaire, ENS de Lyon, Mars.

Isaac S., (2009). Techniques d'animation, Pré-BSQF. 
Jacqueline C., (2003). Apprivoiser les différences, Éditions de la chenelière.

Leeman D., (2002). La construction du sens par la grammaire, Cahiers de l'ILSL, No. 13, Lausanne, PP. 17 - 36.

Leroy-Miquel C., et Goliot-Lété A., (1997). Vocabulaire progressif $d u$ français, clé international, Paris.

Lortie-Paquette M., (1999). Le référentiel pédagogique, un outil pour concrétiser le projet éducatif en classe, pour des pratiques pédagogiques revitalisées, bibliothèque nationale du Québec, Canada.

Lüdi G., (1997). Apprendre à construire des mots : La suffixation nominale chez des apprenants germanophones du français, Basel Electronic Working Papers in Languistics, No. 1, Université de Basel.

Manesse D., (consulté en 2017). De l'école au collège : Enseigner / Apprendre une langue étrangère en $6^{\mathrm{e}}$, journée internationale d'étude de l'équipe INRP, disponible sur www.inrp.fr.

Martinez P., (1998). La didactique des langues étrangères, Que sais-je ?, $2^{\text {ème }}$ édition, PUF, Paris.

Meirieu P., (1989). Apprendre, oui mais

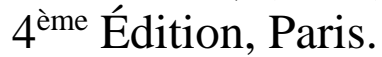
..comment?, E. S. F., Meirieu P., (1990). L'école, mode d'emploi, des méthodes actives à la pédagogie différenciée, $\mathrm{ESF}, 5^{\mathrm{e}}$ éd.

Meyniac J.-P., (2014). Enseigner avec la pédagogie différenciée, Espace Pédagogique, Mois de Mars.

Ministère de la citoyenneté et de l'immigration, (2012). Niveaux de compétence linguistique canadiens : Français langue seconde pour adultes, Centre des niveaux de compétence linguistique canadiens, Ontario, disponible sur www.language.ca.

Miquel C., (2005). Grammaire en dialogues, clé international, Paris. Miquel C., (2007). Vocabulaire en dialogues, clé international, Paris.

Nahla Chellouai, (2013). Difficultés et obstacles dans l'enseignement / apprentissage de la production écrite en FLÉ : Cas des apprenants de la $2^{\text {ème }}$ AS, mémoire de master, Université de Mohammed Kheider de Biskra, Algérie.

Najib Rabadi et Akram Odeh, (2010). L'analyse des erreurs en FLÉ chez les apprenants jordaniens et bahreïniens, Jordan Journal of Modern languages and litterature, Vol. 2, No. 2, PP. 163-177.

Nunes Oliveira D., (2010). Les difficultés des élèves à communiquer à l'oral en classe de $9^{\text {ème }}$ et $10^{\text {ème }}$ au lycée Abilio Duarte de Palmarejo : Réalités et perspectives, mémoire de maîtrise, Université du Cap Vert, Portugal. 
Overmann M., (2009). Histoire des méthodologies, Disponible sur www.ph-ludwigsburg.de/html/2b-frnz-s-

01/overmann/glossaire/méthodologie.html.

Ozcelik N., (2012). L'enseignement et l'apprentissage de la grammaire en classe du FLÉ, la revue de «Ondukoz Mayis», No. 31(1), Université d'«Egitim», Turquie, PP. $175-186$.

Paquette C., (1999). Vers un projet éducatif nouveau, pour des pratiques pédagogiques revitalisées, bibliothèque nationale du Québec, Canada.

Perrenoud Ph., (1993). Échec et soutien scolaire, se former, Voies livres, No. 527, Mai.

Plazaola Giger I., (1996). À propos des méthodes communicatives : la transposition didactique en FLS, bulletin suisse de linguistique appliquée, No. 64, PP. 145-165.

Porquier R., (1977). L'analyse des erreurs : problèmes et perspectives, Études de linguistique appliquée, No. 25, Janvier - Mars, PP. 25 - 43.

Puren C., (2006). De l'approche communicative à la perspective actionnelle, le français dans le monde, No. 347, sept.-oct., PP. 37-40.

Puren C., (1995). Des méthodologies constituées et de leur mise en question, le français dans le monde, Numéro spécial sous le titre «méthodes et méthodologies», Janvier, PP. 36-41.

Puren C., (1988). Histoire des méthodologies de l'enseignement des langues, CLÉ International, Nathan, Paris. Disponible sur www.christianpuren.com.

Puren C., et al., (1998). Se former en didactique des langues, Ellipses, Paris.

Prupas A. et Patella S., (2007). La différenciation pédagogique pour rejoindre tous les élèves, Services Éducatifs et Complémentaires, No. 1, Vol. 1, Commission Scolaire Sir-Wilfrid-Laurier, Novembre. Disponible sur www.webhost.bridgeview.edu.

Richterich, R. et Suter, B., (1981). Cartes sur table, Hachette, paris.

Riquois E., (2010). Évolutions méthodologiques des manuels et matériels didactiques complémentaires en FLÉ : de la méthode traditionnelle à la perspective actionnelle, Éducation \& Formation, e-292, Janvier, Disponible sur www.halshs.archives-ouvertes.fr/halshs-01066557.

Roulet E., (1972). Théories grammaticales, descriptions et enseignement des langues, Labor, Nathan, Paris.

Starets M., (2008). Principes linguistiques en pédagogie des langues : un traité de linguistique appliquée, les presses de l'université Laval, Québec, Canada. 
Starets M., (2000). Théories syntaxiques du français contemporain, Les presses de l'université Laval, Québec, Canada.

Tarif N., (2005). La pédagogie différenciée au service de l'apprentissage, dans le dossier intitulé «approche différenciée et évaluation», Vie pédagogique, No. 134, PP. 21 - 24.

Tisset C., (2010). Enseigner la langue française à l'école : la grammaire, l'orthographe et la conjugaison, Hachette livre. Disponible sur www.hachette-éducation.com.

Tomlinson C., (2004). La classe différenciée, Éditions de la chenelière.

Valverde-Mateos A., (2012). Analyse des erreurs de l'apprentissage de FLÉ basées sur les corpus oraux, Thèse de PHD., Université de Madrid.

Valenzuela O., (2010). La didactique des langues étrangères et les processus d'enseignement / apprentissage, Synergies Chili, No. 6, PP. 7186.

Vicente J., (2016). Typologie des erreurs et des difficultés dans l'apprentissage du français langue étrangère par les hispanophones, Thèse de PHD., Université de Salamanca, Espagne.

Zakhartchouk J.-M., (2001). La pédagogie différenciée renforce-t-elle l'exclusion ?, Cahiers pédagogiques, No. 391.

B) Références en langue anglaise.

Austin J., (1962). How do things with words, Clarendon press, Oxford.

Little D., (1997). Linguistic awareness \& writing : exploring the relationship with language awareness, language awareness, Vol. 6, No. 2-3, PP. 95-104.

Krashen S., (1985). The Input Hypothesis : Issues and Implications, Longman, London.

Krashen S. \& Terrel T., (1983). The Natural Approach: Language Acquisition in the Classroom, Pergamon Press, Oxford.

Simpkins P., et al., (2009). Differentiated curriculum enhancement in inclusive $5^{\text {th }}$ grade science classes, remedial and special education, No. 5, Vol. 30, Sept. - Oct., PP. 300-308.

Tomlinson C., (2003). Differentiating instruction for academic diversity, (Ed.) Cooper J., Classroom teaching skills, $7^{\text {th }}$ ed., Houghton Mifflin, Boston. Available on www.webhost.bridgeview.edu.

Watts-Taffe S., et al., (2012). Differentiated Instruction making informed teacher decisions, the reading teacher, Issue 4, Vol. 66, PP.303314.

C) Références en langue arabe.

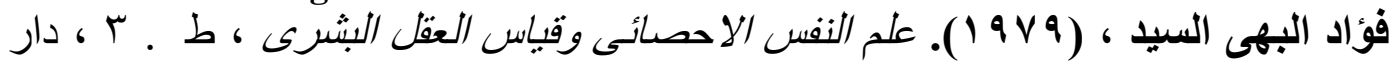

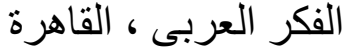

